\title{
Fermionic superoperators for zero-temperature nonlinear transport: Real-time perturbation theory and renormalization group for Anderson quantum dots
}

\author{
R. B. Saptsov ${ }^{1,2}$ and M. R. Wegewijs $1,2,3$ \\ ${ }^{1}$ Peter Grünberg Institut, Forschungszentrum Jülich, 52428 Jülich, Germany \\ ${ }^{2}$ JARA-Fundamentals of Future Information Technology \\ ${ }^{3}$ Institute for Theory of Statistical Physics, RWTH Aachen University, 52056 Aachen, Germany \\ (Received 13 July 2012; revised manuscript received 23 November 2012; published 18 December 2012)
}

\begin{abstract}
We study electron quantum transport through a strongly interacting Anderson quantum dot at finite bias voltage and magnetic field at zero temperature using the real-time renormalization group (RT-RG) in the framework of a kinetic (generalized master) equation for the reduced density operator. To this end, we further develop the general, finite-temperature real-time transport formalism by introducing field superoperators that obey fermionic statistics. This direct second quantization in Liouville Fock space strongly simplifies the construction of operators and superoperators that transform irreducibly under the Anderson-model symmetry transformations. The fermionic field superoperators naturally arise from the univalence (fermion-parity) superselection rule of quantum mechanics for the total system of quantum dot plus reservoirs. Expressed in these field superoperators, the causal structure of the perturbation theory for the effective time-evolution superoperator kernel becomes explicit. Using the constraints of the causal structure, we construct a parametrization of the exact effective time-evolution kernel for which we analytically find the eigenvectors and eigenvalues in terms of a minimal set of only 30 independent coefficients. The causal structure also implies the existence of a fermion-parity protected eigenvector of the exact Liouvillian, explaining a recently reported result on adiabatic driving [Contreras-Pulido et al., Phys. Rev. B 85, 075301 (2012)] and generalizing it to arbitrary order in the tunnel coupling $\Gamma$. Furthermore, in the wide-band limit, the causal representation exponentially reduces the number of diagrams for the time-evolution kernel. The remaining diagrams can be identified simply by their topology and are manifestly independent of the energy cutoff term by term. By an exact reformulation of this series, we integrate out all infinite-temperature effects, obtaining an expansion targeting only the nontrivial, finite-temperature corrections, and the exactly conserved transport current follows directly from the time-evolution kernel. From this new series, the previously formulated RT-RG equations are obtained naturally. We perform a complete one-plus-two-loop RG analysis at finite voltage and magnetic field, while systematically accounting for the dependence of all renormalized quantities on both the quantum dot and reservoir frequencies. Using the second quantization in Liouville space and symmetry restrictions, we obtain analytical RT-RG equations, which can be solved numerically in an efficient way, and we extensively study the model parameter space, excluding the Kondo regime where the one-plus-two-loop approach is obviously invalid. The incorporated renormalization effects result in an enhancement of the inelastic cotunneling peak, even at a voltage $\sim$ magnetic field $\sim$ tunnel coupling $\Gamma$. Moreover, we find a tunnel-induced nonlinearity of the stability diagrams (Coulomb diamonds) at finite voltage, both in the single-electron tunneling and inelastic cotunneling regime.
\end{abstract}

DOI: 10.1103/PhysRevB.86.235432 PACS number(s): 73.63.Kv, 05.10.Cc, 03.65.Yz, 05.60.Gg

\section{INTRODUCTION}

Nonlinear transport spectroscopy of nanoscale systems is a key technique in modern day physics. Although the linear (equilibrium) transport regime is well understood ${ }^{1,2}$ (see Ref. 3 for a review), the theoretical description of nonequilibrium transport, especially at low temperature, remains challenging. Recent progress in this direction has led to an improved understanding of quantum transport through strongly interacting systems (see Ref. 4 for a recent comparative review). Several fully numerical approaches have appeared, such as the scattering-state time-dependent numerical renormalization group ${ }^{5}$ (TD-NRG) relying on the discretization parameter approximation, ${ }^{6}$ time-dependent density matrix renormalization group (TD-DMRG) ${ }^{7-9}$ iterative path integrals (IPSI) ${ }^{10}$ numerically exact influence functional path integrals (INFPI), ${ }^{11,12}$ quantum Monte Carlo (QMC) in combination with the Nakajima-Zwanzig projection technique ${ }^{13}$ or with an imaginary-time formalism, ${ }^{14}$ and diagrammatic Monte Carlo (diagMC). ${ }^{15}$ Partially analytical approaches involve the non- crossing approximation (NCA), ${ }^{16,17}$ equations of motion for Green's functions, ${ }^{18}$ Bethe ansatz, ${ }^{19,20}$ and the flow-equation approach. ${ }^{21}$ Fully analytical approaches include the Keldysh perturbation theory to high orders in the Coulomb interaction $U$ (Refs. 22 and 23) or the dual-fermion superperturbation theory. ${ }^{24}$ Finally, perturbative renormalization group studies have mostly started from the Kondo-model mapping of the Anderson model, either working with Keldysh Green's functions $^{25}$ or the reduced density operator approach. ${ }^{26,27}$

The density operator approach has a long history in various fields of physics and chemistry. In the context of nanoscale transport, it is a natural starting point for the description of systems with large interaction energies in the hightemperature, weak-coupling limit $U \gg T \gg \Gamma$. It can be systematically extended to include high-order tunneling processes using the real-time diagrammatic ${ }^{28}$ or Nakajima-Zwanzig approach $^{29}$ technique, in particular when combined with Liouville space techniques. ${ }^{30}$ However, at low temperature this approach becomes problematic due to the effect of high-energy 
contributions that renormalize the effective low-energy physics. In this paper, we show how this approach can be extended to this regime. In particular, we show that much of the insightful structure of generalized the master/kinetic equation approach at high temperature is preserved in this regime. We formulate a real-time renormalization group approach that naturally connects to the generalized quantum master or kinetic equation approach.

For this purpose, we study the simplest possible benchmark model of an interacting quantum dot (QD) coupled to metallic electrodes, the Anderson model. We explicitly set up a general approach to deal with the nonlinear transport at very low temperature using a renormalization group formulated in the framework of the real-time perturbation theory. We proceed analytically as far as possible, making this a technically challenging task. In fact, the problem is not really manageable without a different physical approach for dealing with superoperators. The development of this Liouville-Fock-space approach is a central topic of this paper. Our approach differs from previous formulations ${ }^{31-34}$ both by its construction and by the scope of its application. The most closely related is that of Prosen, ${ }^{31}$ which was used to calculate steady states of quadratic effective Liouvillians. Here, we extend it to the reservoirs with continuous fields as well and further develop it to simplify the microscopic derivation of effective Liouvillians for nonquadratic problems. The approach introduced by Schmutz ${ }^{32}$ and used by other authors ${ }^{33,34}$ differs from our approach in principal details that are essential for our application. See also the very recent Ref. 35 .

A large part of the paper is devoted to the development of our Liouville-Fock space approach since it allows for general physical insights into the problem and is crucial for overcoming technical difficulties in setting up the real-time renormalization group (RT-RG). We will illustrate its potential in the application to the RT-RG calculation of the nonlinear transport at zero temperature. As a result, this paper by necessity is extensive. Further motivation for its length is that several general physical insights into the real-time approach have not been pointed out, although this approach has been developed for some time and has found widespread use (see Ref. 26 for a review). It is therefore required that the approach is set up from scratch, paying special attention to (i) the causal structure and the related Keldysh rotation, (ii) the Liouville space formulation, in particular the second quantization for superoperators, (iii) the spin- and charge-rotation symmetries, and (iv) the infinite-temperature limit, which serves as a reference point both for the second quantization technique that we develop, as well as for the perturbation theory and RT-RG. Only by fully exploiting these does the application of RT-RG to the Anderson model become feasible. Clearly, these developments are best presented coherently in the context of the application to the RT-RG for which it is absolutely crucial.

To indicate the impact of these developments for our study of the Anderson model, we note that the simplest approximation that includes the exact result for the $U=0$ limit requires an infinite series of diagrams in the standard real-time perturbation theory. Using the RT-RG, this result is recovered only when performing a one- and two-loop analysis for the effective Liouvillian and including one-loop vertex corrections. We emphasize that when applied to the interacting case, this incorporates renormalization effects from strong tunneling, while neglecting spin-fluctuation processes relevant only in the Kondo regime, which enter only in a three-loop $\mathrm{RG}$ analysis (the latter has been addressed previously based on a Kondo-model mapping ${ }^{27}$ ). Naively formulating these RG equations leads to hundreds of nonlinear, coupled integrodifferential equations for frequency-dependent coupling functions. The central result of this paper is the derivation of 30 coupled differential coupling functions, which systematically incorporate the leading frequency dependence and describe the $U=0$ limit exactly. On the way, we derive several exact results of general importance. Altogether, this makes an efficient numerical implementation possible and allows experimentally relevant stability diagrams to be calculated from wide ranges of parameters in the nonlinear zero-temperature regime (excluding the narrow Kondo regime). Many of the results can be extended to generic models involving local interactions (multiorbital Anderson-type models) with bilinear tunnel coupling to reservoirs. See Ref. 36 for a recent study of nonlocal interactions using the RT-RG.

The paper is organized in three main parts as follows. In the first part, Sec. II, we formulate the model and directly revert to a Liouville space description and develop the kinetic equation approach for the stationary QD density operator. We formulate the perturbation series for the effective Liouvillian $L(z)$ appearing in this equation using what we will call the causal representation of field superoperators $G$ with fermionic statistics. We emphasize that this formulation of the perturbation theory, although equivalent to previous formulations, ${ }^{29,30,37}$ leads to many simplifications beyond the application of interest here, and therefore warrants a proper, extensive discussion. Several of these results have already found application ${ }^{38}$ and even provide insights into, and generalization of, recent interesting predictions. ${ }^{39}$ Moreover, a renormalized perturbation theory that takes the infinitetemperature limit as a formal reference point suggests itself. It also connects in a natural way to the renormalization group approach while preserving much of its general perturbative structure. We show how the calculation of the current requires little additional calculation and prove that in our nonlinear approach the linear current vanishes at zero bias, a fact that is not obvious from the general structure of the theory.

In the second main part of the paper, Sec. III, the explicit one- and two-loop RT-RG equations are derived, accounting for the energy dependence of both the Liouvillian and the vertices due to the finite nonlinear transport voltage. For the noninteracting case $U=0$, the current is shown to arise naturally as an exact result already in the one-loop RT-RG, implying that all two-loop corrections to this observable arise from the strong local Coulomb interaction. We find, however, that for $U=0$ nonzero, two-loop terms exist, which are relevant when one is interested in, e.g., the density matrix (and not just the current). The nontrivial frequency dependence of two-loop equations is systematically accounted for in powers of the renormalized dimensionless coupling superoperators $\bar{G}$, resulting in an effective RG equation for an effective Liouvillian only that accounts for vertex renormalization corrections.

This simplification enables the detailed numerical study of the zero-temperature, nonlinear transport in the third part of the paper, Sec. IV. This covers all regimes, except for 
the Kondo regime of low applied voltage and magnetic field. The importance of accounting for both one- and twoloop corrections, as well as the nonequilibrium Matsubara axes, is demonstrated numerically. Finally, we show that the tunnel-induced renormalization effects incorporated in our one-plus-two-loop approach enhance the inelastic cotunneling resonance at finite magnetic field and voltage and generate nonlinearities of the single-electron tunneling (SET) stability diagrams (Coulomb diamonds).

\section{MODEL AND REAL-TIME TRANSPORT THEORY}

\section{A. Anderson model}

In this section, we introduce the model and our compact notation, which is crucial to the Liouville space formulation of the theory. The simplest model Hamiltonian of a QD that takes into account Coulomb interaction effects involves just a single orbital:

$$
H=\epsilon n+B S_{z}+U n_{\uparrow} n_{\downarrow},
$$

where $n=\sum_{\sigma} n_{\sigma}$ and $n_{\sigma}=d_{\sigma}^{\dagger} d_{\sigma}$ are the occupation operators. Here, $\epsilon$ denotes the energy of the orbital, experimentally controlled by the gate voltage $V_{g}$ (we take $\epsilon=-V_{g}$ ), and $U$ is the Coulomb charging energy. The index $\sigma= \pm$ corresponds to spin up ( $\uparrow)$ and down $(\downarrow)$ and $S_{z}=\frac{1}{2} \sum_{\sigma} \sigma n_{\sigma}$ is the $z$ component of the spin vector operator $\mathbf{S}=\sum_{\sigma \sigma^{\prime}} \frac{1}{2} \boldsymbol{\sigma}_{\sigma, \sigma^{\prime}} d_{\sigma}^{\dagger} d_{\sigma^{\prime}}$ along the external magnetic field $\mathbf{B}=B \mathbf{e}_{z}$ (in units where $\left.g \mu_{B}=1\right)$ and $\sigma$ is the vector of Pauli matrices. The dot is attached to electrodes, treated as free electron reservoirs:

$$
H^{R}=\sum_{\sigma, r, k} \epsilon_{r, k} a_{\sigma, r, k}^{\dagger} a_{\sigma, r, k},
$$

where the index of the spin $\sigma= \pm$, quantized along the $z$ axis, corresponds to $\uparrow, \downarrow$, the reservoir index $r= \pm$ corresponds to $L, R$, and $k$ is the orbital index. The reservoir electron number and spin operator can be decomposed as $n^{R}=\sum_{r} n^{r}$ and $\mathbf{s}^{R}=\sum_{r} \mathbf{s}^{r}$, respectively, into

$$
\begin{aligned}
& n^{r}=\sum_{\sigma, k} a_{\sigma, r, k}^{\dagger} a_{\sigma, r, k}, \\
& \mathbf{s}^{r}=\sum_{\sigma, k} \frac{1}{2} \sigma_{\sigma, \sigma^{\prime}} a_{\sigma, r, k}^{\dagger} a_{\sigma^{\prime}, r, k} .
\end{aligned}
$$

In the continuum limit, the reservoirs are described by the density of states $\nu_{r}(\omega)=\sum_{k} \delta\left(\omega-\epsilon_{r, k}+\mu_{r}\right)$ and we go to the energy representation of the fermionic operators

$$
a_{\sigma, r}(\omega)=\frac{1}{\sqrt{v_{r}(\omega)}} \sum_{k} a_{\sigma, r, k} \delta\left(\omega-\epsilon_{r, k}+\mu_{r}\right),
$$

with the anticommutation relations

$$
\begin{gathered}
{\left[a_{\sigma, r}(\omega), a_{\sigma^{\prime}, r^{\prime}}^{\dagger}\left(\omega^{\prime}\right)\right]_{+}=\delta_{\sigma, \sigma^{\prime}} \delta_{r, r^{\prime}} \delta\left(\omega-\omega^{\prime}\right),} \\
{\left[a_{\sigma, r}(\omega), a_{\sigma^{\prime}, r^{\prime}}\left(\omega^{\prime}\right)\right]_{+}=0,}
\end{gathered}
$$

where we denote (anti)commutators by $[A, B]_{ \pm}=A B \pm B A$. Thus, we have for the reservoir Hamiltonian

$$
H^{\mathrm{R}}=\sum_{\sigma, r} \int d \omega\left(\omega+\mu_{r}\right) a_{\sigma, r}^{\dagger}(\omega) a_{\sigma, r}(\omega) .
$$

In contrast to $\epsilon_{r, k}$, the energy $\omega$ is the electron energy relative to $\mu_{r}$, i.e., the reference energy depends on which reservoir $r$ is considered.

The junctions connecting the dot and reservoirs are modeled by the tunneling Hamiltonian

$$
\begin{gathered}
V=\sum_{r} V^{r} \\
V^{r}=\sum_{\sigma} \int d \omega \sqrt{v_{r}(\omega)}\left[t_{r}(\omega) a_{\sigma, r}^{\dagger}(\omega) d_{\sigma}+\text { H.c. }\right] .
\end{gathered}
$$

The Hamiltonian of the total system is denoted by

$$
H^{\mathrm{tot}}=H+H^{\mathrm{R}}+V .
$$

We assume $t_{r}(\omega)$ to be real and introduce the spectral density

$$
\Gamma_{r}(\omega)=2 \pi v_{r}(\omega)\left|t_{r}(\omega)\right|^{2}
$$

and rescaled field operators

$$
b_{\sigma, r}(\omega)=\sqrt{\frac{\Gamma_{r}(\omega)}{2 \pi}} a_{\sigma, r}(\omega) .
$$

To make the notation more compact, we introduce an additional particle-hole index $\eta$ (Ref. 40):

$$
\begin{gathered}
b_{\eta, \sigma, r}(\omega)= \begin{cases}b_{\sigma, r}^{\dagger}(\omega) & \eta=+, \\
b_{\sigma, r}(\omega) & \eta=-\end{cases} \\
d_{\eta, \sigma}= \begin{cases}d_{\sigma}^{\dagger} & \eta=+, \\
d_{\sigma} & \eta=-.\end{cases}
\end{gathered}
$$

Throughout the paper, we will denote the inverse value of a two-valued index with a bar, e.g.,

$$
\bar{\eta}=-\eta \text {. }
$$

We combine all indices into a multi-index variable written as a number:

$$
1=\eta, \sigma, r, \omega, \quad \overline{1}=\bar{\eta}, \sigma, r, \omega .
$$

By way of exception, the bar denotes inversion of the particlehole index $\eta$ only. With $b_{1}=b_{\eta, \sigma, r}(\omega)$ and $b_{\overline{1}}=b_{-\eta, \sigma, r}(\omega)$, the various independent anticommutation relations can be compactly summarized as

$$
\begin{gathered}
{\left[d_{1}, d_{2}\right]_{+}=\delta_{1 \overline{2}},} \\
{\left[b_{1}, b_{2}\right]_{+}=\frac{\Gamma_{1}}{2 \pi} \delta_{1 \overline{2}},}
\end{gathered}
$$

where $\Gamma_{1}=\Gamma_{r}(\omega)$. The interaction then simply reads as

$$
V=b_{\overline{1}} d_{1},
$$

where we implicitly assume summation over all discrete parts of the multi-index 1 (i.e., $\eta, \sigma, r$ ) and integration over its continuous part $(\omega)$. If we have more than one multi-index, we distinguish their components by corresponding subscripts: $1=\eta_{1}, \sigma_{1}, r_{1}, \omega_{1}, 2=\eta_{2}, \sigma_{2}, r_{2}, \omega_{2}$. We will often omit these subscripts if only one multi-index appears. Importantly, it can be shown ${ }^{41}$ that operators (and, below also superoperators) of the dot and the reservoirs can be treated as if they commute (rather than anticommute), i.e., $\left[d_{1}, b_{2}\right]_{-}=0$ for all multi-indices 1,2 .

The reservoirs are assumed to be in thermal equilibrium, each described by its own grand-canonical density operator 
with temperature $T$ and electrochemical potential $\mu^{r}$ :

$$
\rho^{\mathrm{R}}=\prod_{r} \rho^{r}, \quad \rho^{r}=\frac{1}{z^{r}} e^{-\frac{1}{T}\left(H^{r}-\mu^{r} n^{r}\right)},
$$

where $z^{r}=\operatorname{Tr}_{r} e^{-\frac{1}{T}\left(H^{r}-\mu^{r} n^{r}\right)}$. We assume that a symmetric bias is applied to the electrodes, i.e., $\mu_{L, R}= \pm V / 2$. We note the key property

$$
b_{1} \rho^{\mathrm{R}}=e^{\eta_{1} \omega_{1} / T} \rho^{\mathrm{R}} b_{1} .
$$

In Eq. (12), the density of states varies on the energy scale of the bandwidth $D$, which we assume to be much larger than any other energy scale in the problem. In this wide-band limit, we can assume $\Gamma_{r}(\omega)$ to be energy independent and cut off all reservoir energy integrals $(\omega)$ at the scale $D$. The detailed energy dependence of $\Gamma_{r}(\omega)$ at high energies is not crucially important for the results. ${ }^{26}$ In the actual applications in Sec. IV, we will assume for simplicity that the tunnel couplings are symmetric, i.e., $\Gamma_{L}=\Gamma_{R}=\Gamma$, and consider the low-temperature limit, i.e., $T \ll U, V, \Gamma_{r}$, by setting $T=0$. The results of this section and much of Sec. III, however, do not depend on these assumptions unless explicitly indicated.

For a nonzero magnetic field $B$ and finite Coulomb interaction $U$, the total system possesses two locally and globally conserved quantities that will play an important role. The charge and the spin components along the magnetic field are conserved individually in the dot and in the reservoirs

$$
\begin{gathered}
{[H, n]_{-}=0, \quad\left[H, S_{z}\right]_{-}=0,} \\
{\left[H^{\mathrm{R}}, n^{\mathrm{R}}\right]_{-}=0, \quad\left[H^{\mathrm{R}}, S_{z}^{\mathrm{R}}\right]_{-}=0 .}
\end{gathered}
$$

These conservation laws extend to the total charge $N^{\text {tot }}=n+$ $n^{\mathrm{R}}$ and spin $S_{z}^{\text {tot }}=S_{z}+S_{z}^{\mathrm{R}}$ since the interaction $V$ commutes with these operators:

$$
\begin{aligned}
& {\left[H^{\mathrm{tot}}, N^{\mathrm{tot}}\right]_{-}=0,} \\
& {\left[H^{\mathrm{tot}}, S_{z}^{\mathrm{tot}}\right]_{-}=0 .}
\end{aligned}
$$

\section{B. Density operator and diagram rules}

The purpose of this section is twofold. First, we briefly review the real-time approach to the calculation of the stationary reduced density operator, introducing the central quantities $\Sigma(z)$, the self-energy superoperator, and $L(z)$, the effective Liouvillian, and their perturbative expansions in vertex superoperators $G$. Second, we introduce a "causal" representation of the perturbation theory, which allows for a compact formulation and derivation of the diagrammatic rules for the self-energy $\Sigma$. Moreover, many general physical insights become explicitly clear in this representation. In particular, this new formulation naturally suggests the possibility of a two-stage, real-time renormalization group (RT-RG), which will be set up in Secs. II B4 and III.

\section{Stationary density operator}

In order to find the QD stationary state, we need to consider the evolution of the total system density operator. It evolves according to the Liouville-von Neumann equation

$$
\partial_{t} \rho^{\mathrm{tot}}(t)=-i\left[H^{\mathrm{tot}}, \rho^{\mathrm{tot}}(t)\right]_{-}=-i L^{\mathrm{tot}} \rho^{\mathrm{tot}}(t),
$$

with the superoperator Liouvillian $L^{\text {tot }}=\left[H^{\text {tot }}, \bullet\right]_{-}$. Superoperators are linear transformations of operators and throughout the paper we let the solid bullet $(\bullet)$ indicate the operator on which a superoperator acts (if needed). Explicit matrix representations of superoperators are only required for the QD part and will be discussed later on in Sec. II C.

The initial state of the total system at the initial time $t_{0}$ is assumed to be the direct product of the dot density matrix and the equilibrium density matrices (21) of the electrodes:

$$
\rho^{\text {tot }}\left(t_{0}\right)=\rho\left(t_{0}\right) \rho^{\mathrm{R}} \text {. }
$$

We will discuss some properties of $\rho\left(t_{0}\right)$ further in the following. The formal solution of Eq. (27) is

$$
\begin{aligned}
\rho^{\mathrm{tot}}(t) & =e^{-i H^{\mathrm{tot}}\left(t-t_{0}\right)} \rho^{\mathrm{tot}}\left(t_{0}\right) e^{i H^{\mathrm{tot}}\left(t-t_{0}\right)} \\
& =e^{-i L^{\mathrm{tot}}\left(t-t_{0}\right)} \rho^{\mathrm{tot}}\left(t_{0}\right) .
\end{aligned}
$$

The reduced density matrix of the dot is obtained by integrating out the reservoir degrees of freedom:

$$
\rho(t)=\operatorname{Tr}_{\mathrm{R}} \rho^{\mathrm{tot}}(t)=\operatorname{Tr}_{\mathrm{R}}\left(e^{-i L^{\mathrm{tot}}\left(t-t_{0}\right)} \rho\left(t_{0}\right) \rho^{\mathrm{R}}\right) .
$$

We now decompose $L^{\text {tot }}=L+L^{\mathrm{R}}+L^{V}$, where $L=[H, \bullet]_{-}$ and $L^{\mathrm{R}}=\left[H^{\mathrm{R}}, \bullet\right]_{-}$, and set up the perturbation series in the tunnel coupling $L^{V}=[V, \bullet]_{-} \sim \sqrt{\Gamma}$. It is then more convenient ${ }^{26}$ to use the Laplace transform of the dot reduced density matrix for $\operatorname{Im} z>0$ :

$$
\begin{aligned}
\rho(z) & =\int_{t_{0}}^{\infty} d t e^{i z\left(t-t_{0}\right)} \rho(t) \\
& =\operatorname{Tr}_{\mathrm{R}}\left(\frac{i}{z-L-L^{\mathrm{R}}-L^{V}} \rho^{\mathrm{tot}}\left(t_{0}\right)\right) .
\end{aligned}
$$

We will refer to $z$ as the dot frequency. We expand the resolvent in $L^{V}$, resulting in a geometric series with terms of the form

$$
\operatorname{Tr}_{\mathrm{R}}\left(\frac{1}{z-L-L^{\mathrm{R}}} L^{V} \ldots L^{V} \frac{1}{z-L-L^{\mathrm{R}}} \rho^{\mathrm{tot}}\left(t_{0}\right)\right) .
$$

The average over the reservoirs can now be calculated directly using a Wick theorem for field superoperators [see Eq. (60) and Appendix A). Collecting irreducible contractions into the selfenergy superoperator $\Sigma(z)$ (see Sec. II B3), the perturbation series can be resummed to

$$
\rho(z)=\frac{i}{z-L(z)} \rho\left(t_{0}\right),
$$

where we have introduced the effective dot Liouvillian

$$
L(z)=L+\Sigma(z) .
$$

To keep the notation to a minimum, we distinguish this quantity from the "bare" dot Liouvillian $L=[H, \bullet]_{-}$by simply appending the dependence on the frequency $z . L(z)$ completely determines the time evolution of the reduced density operator. A key idea exploited both in the perturbation theory and in the renormalization group is that one is free to modify $L$ and $\Sigma(z)$ as long as their sum remains equal to $L(z)$. The equation determining the stationary density matrix $\rho=\lim _{t-t_{0} \rightarrow \infty} \rho(t)=\lim _{z \rightarrow i 0}(-i z) \rho(z)$ is now obtained by multiplying Eq. (35) by $-i z[z-L(z)]$ and taking $z \rightarrow i 0$ :

$$
L(i 0) \rho=0 \text {. }
$$


Before deriving the perturbation series for $\Sigma(z)$ in Sec. II B3, we first introduce a convenient representation of the field superoperators.

\section{Causal representation of fermionic field superoperators}

In the following, we integrate out explicitly the reservoir degrees of freedom while keeping track of those of the QD. To facilitate this, the tunnel coupling superoperator $L^{V}=[V, \bullet]_{-}$ should be written as a convenient product of superoperators of the dot and the reservoirs: inserting Eq. (20), we have

$$
L^{V}=p^{L^{N^{\text {tot }}}} p \mathscr{J}_{\overline{1}}^{p} \mathscr{G}_{1}^{p},
$$

where we defined the following "naive" field superoperators:

$$
\begin{gathered}
\mathscr{J}^{p} \bullet= \begin{cases}b_{1} \bullet & p=+, \\
\bullet b_{1} & p=-,\end{cases} \\
\mathscr{G}^{p} \bullet= \begin{cases}d_{1} \bullet & p=+, \\
\bullet d_{1} & p=-.\end{cases}
\end{gathered}
$$

The superscript $p= \pm$ keeps track of whether a field operator acts from the left or right and is referred to as the Keldysh index by analogy to the Green's function and functional integral techniques. In Eq. (38), we implicitly sum over $p$, in addition to the multi-index 1 . A crucial difference to the formulation of Ref. 26 is that we introduced a harmless additional superoperator



into Eq. (38), where

$$
\begin{gathered}
L^{N^{\text {tot }}}=\left[N^{\text {tot }}, \bullet\right]_{-}=L^{n}+L^{n^{\mathrm{R}}}, \\
L^{n}=[n, \bullet]_{-} \\
L^{n^{\mathrm{R}}}=\left[n^{\mathrm{R}}, \bullet\right]_{-}
\end{gathered}
$$

are the superoperators associated with the total, QD, and reservoir electron numbers, respectively. Clearly, for $p=+1$ this factor is trivially equal to 1 . However, it may seem at first sight that for $p=-1$ this is not the case: when applied to a projector of states of the total system, this superoperator counts the relative parity of the fermion numbers $N$ and $N^{\prime}$ : $(-1)^{L^{N^{\text {tot }}}}|N \lambda\rangle\left\langle N^{\prime} \lambda^{\prime}|= \pm| N \lambda\right\rangle\left\langle N^{\prime} \lambda^{\prime}\right|$ for $N-N^{\prime}=$ even/odd, where $\lambda$ and $\lambda^{\prime}$ denote further quantum numbers. However, in all calculations we can assume that the total-system density operators on which it acts have even parity since odd fermionparity components of states can neither be measured by any physical operator nor be prepared using physical evolutions. This is referred to as the fermion-parity superselection rule of quantum mechanics. ${ }^{42,43}$

Since the fermion parity plays an important role in what follows but is often not mentioned or used explicitly in density operator approaches, it warrants some discussion, in particular since odd-fermion-parity operators do appear in the renormalization group approach. Physical Hamiltonians and observables (and their corresponding superoperators) always contain only products of even numbers of fermionic operators. This implies that only the part of the density operator $\rho^{\text {tot }}(t)$ with even fermion parity can enter into the calculation of any physical observable $\langle A\rangle(t)=\operatorname{Tr} A \rho^{\text {tot }}(t)$. This even part of $\rho^{\text {tot }}(t)$ is generated solely from the even parity part of $\rho^{\text {tot }}\left(t_{0}\right)$ at earlier times since, by the same token, the parity of the total fermion number is conserved during time evolution. Therefore, only the even-fermion-parity part of the initial state $\rho^{\text {tot }}\left(t_{0}\right)$ can contribute to an observable, and one may set any odd-fermion part of any density matrix equal to zero. As a result, we can take in Eq. (38) $p^{L^{N^{\text {tot }}}} \bullet=1 \bullet$, even for $p=-1$. For the factorized initial state Eq. (28) that we assumed here, this implies that $\rho\left(t_{0}\right)$ must be assumed to be of even fermion parity since $\rho^{\mathrm{R}}$ also has even fermion parity $\left[L_{n^{\mathrm{R}}} \rho^{\mathrm{R}}=\left[n^{\mathrm{R}}, \rho^{\mathrm{R}}\right]_{-}=0\right.$, which follows from Eq. (21)].

The useful implications of this fermion-parity conservation become clear when performing a linear transformation of the field superoperators with respect to their Keldysh indices $p$. The naively chosen field superoperators (39) and (40) have the disadvantage that they commute or anticommute depending on the Keldysh index $p$ :

$$
\begin{aligned}
\mathscr{G}_{1}^{p} \mathscr{G}_{2}^{p^{\prime}}+p p^{\prime} \mathscr{G}_{1}^{p} \mathscr{G}_{2}^{p^{\prime}} & =\delta_{p p^{\prime}} \delta_{1 \overline{2}}, \\
\mathscr{J}_{1}^{p} \mathscr{J}_{2}^{p^{\prime}}+p p^{\prime} \mathscr{J}_{1}^{p} \mathscr{J}_{2}^{p^{\prime}} & =\delta_{p p^{\prime}} \delta_{1 \overline{2}} .
\end{aligned}
$$

This complicates many calculations as noted, e.g., in Ref. 44. However, the factorization of the total fermion parity into a dot and reservoir factor in Eq. (41) naturally suggests a transformation of the field operators. By absorbing the fermion-parity superoperators of each subsystem into new field superoperators

$$
\begin{aligned}
\mathcal{G}_{1}^{p} & =p^{L^{n}} \mathscr{G}_{1}^{p}, \\
\mathcal{J}_{1}^{p} & =p^{L^{n^{\mathrm{R}}}} \mathscr{J}_{1}^{p},
\end{aligned}
$$

the latter obey definite anticommutation relations

$$
\begin{aligned}
{\left[\mathcal{G}_{1}^{p}, \mathcal{G}_{2}^{p^{\prime}}\right]_{+} } & =p \delta_{p p^{\prime}} \delta_{1 \overline{2}}, \\
{\left[\mathcal{J}_{1}^{p}, \mathcal{J}_{2}^{p^{\prime}}\right]_{+} } & =\frac{\Gamma}{2 \pi} p \delta_{p p^{\prime}} \delta_{1 \overline{2}} .
\end{aligned}
$$

This allows one to prove the Wick theorem directly for the operators $\mathcal{J}$ using simple algebra, ${ }^{38}$ avoiding the need to carefully keep track of sign factors as done in Ref. 26. However, the nonvanishing anticommutators still depend on the Keldysh index $p$ on the right-hand side. Moreover, the fields have no simple Hermitian superconjugation relation (see Appendix B). This can be avoided by a rotation of the QD fields

$G_{1}^{q}=\left\{\begin{array}{l}\tilde{G}_{1}=\frac{1}{\sqrt{2}} \sum_{p} \mathcal{G}_{1}^{p}=\frac{1}{\sqrt{2}} \sum_{p} p^{L^{n}} \mathscr{G}_{1}^{p} \quad q=+, \\ \bar{G}_{1}=\frac{1}{\sqrt{2}} \sum_{p} p \mathcal{G}_{1}^{p}=\frac{1}{\sqrt{2}} \sum_{p} p^{L^{n}+1} \mathscr{G}_{1}^{p} q=-\end{array}\right.$

and a contravariant rotation (cf. Sec. II B3) of the reservoir fields

$$
J_{1}^{q}=\left\{\begin{array}{l}
\tilde{J}_{1}=\frac{1}{\sqrt{2}} \sum_{p} p \mathcal{J}_{1}^{p}=\frac{1}{\sqrt{2}} \sum_{p} p^{L^{n^{\mathrm{R}}}+1} \mathscr{J}_{1}^{p} q=+, \\
\bar{J}_{1}=\frac{1}{\sqrt{2}} \sum_{p} \mathcal{J}_{1}^{p}=\frac{1}{\sqrt{2}} \sum_{p} p^{L^{n^{\mathrm{R}}}} \mathscr{J}_{1}^{p} q=-.
\end{array}\right.
$$

To facilitate later discussions, we introduced both an index $q=$ \pm as well as "tilde" and "overbar" symbols to distinguish the new field components. Now, the anticommutation relations are 
completely analogous to those of the usual fermionic operators

$$
\left[G_{1}^{q}, G_{1^{\prime}}^{q^{\prime}}\right]_{+}=\delta_{q, \bar{q}^{\prime}} \delta_{1, \overline{1}^{\prime}},\left\{\begin{array}{l}
{\left[\tilde{G}_{1}, \bar{G}_{1^{\prime}}\right]_{+}=\delta_{1, \overline{1}^{\prime}},} \\
{\left[\tilde{G}_{1}, \tilde{G}_{1^{\prime}}\right]_{+}=0,} \\
{\left[\bar{G}_{1}, \bar{G}_{1^{\prime}}\right]_{+}=0}
\end{array}\right.
$$

whereas in the reservoirs we incorporate the coupling into the normalization factor

$$
\left[J_{1}^{q}, J_{1^{\prime}}^{q^{\prime}}\right]_{+}=\frac{\Gamma_{1}}{2 \pi} \delta_{q, \bar{q}^{\prime}} \delta_{1, \overline{1}^{\prime}},\left\{\begin{array}{l}
{\left[\tilde{J}_{1}, \bar{J}_{1^{\prime}}\right]_{+}=\frac{\Gamma_{1}}{2 \pi} \delta_{1, \overline{1}^{\prime}},} \\
{\left[\tilde{J}_{1}, \tilde{J}_{1^{\prime}}\right]_{+}=0,} \\
{\left[\bar{J}_{1}, \bar{J}_{1^{\prime}}\right]_{+}=0 .}
\end{array}\right.
$$

This second transformation is known as the Keldysh rotation ${ }^{45}$ and is, e.g., applied to fermionic fields represented by Grassmann numbers in functional integral theories ${ }^{46}$ or in the Green's-function formalism. ${ }^{45}$ Here, we find that it also considerably simplifies the real-time transport theory in which not all degrees of freedom can be integrated out, in contrast to the cited approaches. We note that a transformation similar to Eqs. (47) and (48), which also results in the usual anticommutation and conjugation relations for the fermionic superoperators, was introduced in Refs. 32-34, however, without performing the Keldysh rotation. This transformation, however, is less convenient since it does not reveal a general structure of the fermionic superoperators that is important for our applications. In the following, this will be related to causality and we will therefore refer to (51) and (52) as the causal representation and to the index $q= \pm$ as the causal index. Again, we denote its inverse by $\bar{q}=-q$. The superoperator approach introduced by Prosen ${ }^{31}$ is related to ours, but is constructed in a different way by first introducing a Fock basis (see Sec. II C2). This construction does not allow one to easily see the relations between Prosen's superoperators and our causal superoperators (51). See our detailed comparison of the existing approaches in Appendixes C and D. For the dot field superoperators $\left(G^{q}\right)$, this representation was already introduced in Ref. 26, but it was not exploited in the context of the perturbation theory, where it provides many useful additional simplifications that we now address.

First, the property that for any multi-index 1,

$$
\underset{D}{\operatorname{Tr}}\left(\bar{G}_{1} \bullet\right)=0
$$

ensures the probability conservation on the dot during the time evolution [see Eq. (69)]. This property of $\bar{G}_{1}$ is extremely important for the formulation of the RT-RG in Sec. III and is preserved during renormalization. We note that probability conservation in the Liouville approach corresponds to the normalization conservation for the partition function in the path-integral approach. ${ }^{46}$ Equation (55) follows directly from the definition of the causal representation: using $\operatorname{Tr}_{D} L^{n}=0$, and, using the cyclic property of the trace, we see that $\operatorname{Tr}_{D} \mathscr{G}_{1}^{p} \bullet=\operatorname{Tr}_{D} d_{1} \bullet$ is independent of $p= \pm$. Therefore,

$$
\underset{D}{\operatorname{Tr}}(\bar{G} \bullet)=\sum_{p} \operatorname{Tr}_{D} p^{L^{n}+1} \mathscr{G}_{1}^{p} \bullet=\sum_{p} p \underset{D}{\operatorname{Tr} d_{1}} \bullet=0 .
$$

Second, the fields $\bar{G}_{1}$ and $\tilde{G}_{\overline{1}}$ are related by Hermitian conjugation of superoperators:

$$
\bar{G}_{1}=\left(\tilde{G}_{\overline{1}}\right)^{\dagger} .
$$

For a superoperator $S$, the Hermitian conjugate $S^{\dagger}$ is defined by $\operatorname{Tr}\left(A^{\dagger} S B\right)=\operatorname{Tr}\left[\left(S^{\dagger} A\right)^{\dagger} B\right]$ where $A$ and $B$ are arbitrary operators (see also Sec. II C1 and Appendix B). This indicates that the conjugate fields $\bar{G}_{1}$ and $\tilde{G}_{\overline{1}}$ are similar to usual creation and annihilation operators, respectively. In Sec. II C, we exploit this "second quantization" in Liouville space to construct a convenient basis for this space that includes the left and right zero eigensupervectors of both vertices. This considerably simplifies the matrix representations of many superoperators required in the perturbation theory and the RG. An immediate consequence of the probability conservation property (55) and the property (57) is that $\tilde{G}$ has the unit operator $\mathbb{1}$ as a right zero eigensupervector:

$$
\tilde{G}_{1} \mathbb{1}=0 .
$$

The above properties of the causal field superoperators provide explicit insight into important physical issues that are otherwise not obvious in the general form of the perturbation theory, e.g., the wide-band limit, the infinite-temperature limit, and the dependence on the energy cutoff $D$, which we discuss in Sec. II B3. Introducing the corresponding causal representation for the reservoir field superoperators also yields several simplifications, which we now discuss.

a. Wick theorem. First, we note that due to the local interactions on the QD, Eq. (53) can not be used to formulate a Wick theorem for the vertices $G$. In contrast to this, for the reservoir field superoperators, this is possible due to the relation

$$
\bar{J}_{1} \rho^{\mathrm{R}}=\tanh \left(\eta_{1} \omega_{1} / 2 T\right) \tilde{J}_{1} \rho^{\mathrm{R}} .
$$

This result follows from Eq. (22) by writing it in superoperator notation $\mathscr{J}_{1}^{+} \rho^{\mathrm{R}}=e^{\eta_{1} \omega_{1} / T} \mathscr{J}_{1}^{-} \rho^{\mathrm{R}}$, and then applying the transformations (48) and (52). With Eq. (59) we can algebraically prove the Wick theorem for the superoperators $J_{1}^{q}$ in close analogy to the usual case of fermionic operators (see Appendix A): the average of a product equals the product of pair contractions summed over all contractions of pairs $\langle i k\rangle$ :

$$
\operatorname{Tr}_{\mathrm{R}}\left(J_{1}^{q_{1}} \ldots J_{m}^{q_{m}} \rho^{\mathrm{R}}\right)=\sum_{i<k} \prod_{\langle i k\rangle}(-1)^{P}\left\langle J_{i}^{q_{i}} J_{k}^{q_{k}}\right\rangle_{\mathrm{R}},
$$

with the usual fermionic sign $(-1)^{P}$ of the permutation $P$ that disentangles the contractions.

b. Causal structure. The number of possible pair contractions appearing on the right-hand side of Eq. (60) is strongly limited by the causal structure. Applying $\operatorname{Tr}_{\mathrm{R}} \bar{J}_{1} \bullet$ to Eq. (59) written for the operator $\bar{J}_{2}$ we obtain a relation between two of the possible four pair contractions:

$$
\left\langle\bar{J}_{1} \bar{J}_{2}\right\rangle_{\mathrm{R}}=\tanh \left(\eta_{2} \omega_{2} / 2 T\right)\left\langle\bar{J}_{1} \tilde{J}_{2}\right\rangle_{\mathrm{R}} .
$$

This is actually a statement of the equilibrium fluctuationdissipation theorem for each reservoir separately (see, e.g., Ref. 47). In close analogy to Eq. (55), one proves that

$$
\operatorname{Tr}\left(\tilde{J}_{1} \bullet\right)=0 .
$$

This implies, in particular, $\left\langle\tilde{J}_{1} \tilde{J}_{2}\right\rangle_{\mathrm{R}}=0$, which is related to the well-known fact that of the four reservoir Green's functions, only three are independent. ${ }^{48}$ This is analogous to the absence of the so-called "quantum-quantum" contractions and of the "classical-classical" term in the action in the Keldysh 
functional integral approach ${ }^{46}$ and has been referred to as the "causal" structure of the Green's functions. However, Eq. (62) entails an additional simplification: $\left\langle\tilde{J}_{1} \bar{J}_{2}\right\rangle_{\mathrm{R}}=0$. This is particular to our real-time superoperator approach, and is most explicitly related to causality. To see this, note that we keep track of the left and right action of operators using superoperator notation, and that these superoperators inherit their ordering in the Laplace representation from their forward time ordering in the time evolution [cf. Eq. (34)]. We never have to introduce a fictitious backward time propagation as in the Keldysh technique. Therefore, no advanced reservoir Green's functions can appear in our theory, which is expressed by $\left\langle\tilde{J}_{1} \bar{J}_{2}\right\rangle_{\mathrm{R}}=0$.

c. Energy and temperature dependence. The only reservoir correlation functions that can appear in the Wick expansion (60) for the causal fields have a simpler energy $(\omega)$ and temperature $(T)$ dependence than in the representations (39), (40), (47), and (48). This correlates with the physical information incorporated in these functions, i.e., in the retarded function $^{49}$

$$
\tilde{\gamma}_{1,2}\left(\eta_{2} \omega_{2}\right):=\left\langle\bar{J}_{1} \tilde{J}_{2}\right\rangle_{\mathrm{R}}=\frac{\Gamma_{2}}{2 \pi} \delta_{1, \overline{2}}
$$

and in the Keldysh function

$$
\bar{\gamma}_{1,2}\left(\eta_{2} \omega_{2}\right):=\left\langle\bar{J}_{1} \bar{J}_{2}\right\rangle_{\mathrm{R}}=\frac{\Gamma_{2}}{2 \pi} \tanh \left(\eta_{2} \omega_{2} / 2 T\right) \delta_{1, \overline{2}} .
$$

In the causal representation, the superoperator ordering explicitly shows that the retarded contraction $\tilde{\gamma}$ contains no information about the distribution function of the reservoirs and is therefore temperature independent. Equation (63) follows directly from the anticommutation relation (54) and the property (62), and is indeed independent of the reservoir density operator $\rho^{\mathrm{R}}$. In contrast, for the Keldysh contraction $\bar{\gamma}$, one first needs to use the property (59) specific to the equilibrium state of the noninteracting reservoirs before Eqs. (54) and (62) can be applied. The above shows that the representation (52) reflects most explicitly the causal structure of the perturbation theory, motivating its denotation. It thereby automatically achieves the decomposition of the reservoir Fermi distribution function into its symmetric (trivial) and antisymmetric (nontrivial) parts with respect to the energy $\omega$ that was introduced in Ref. 26, where it was crucial for setting up the RT-RG. In the following, we show that in the causal representation for the QD fields, the perturbation theory also drastically simplifies and that in this formulation the RT-RG appears quite naturally.

\section{Perturbation series and diagram rules}

a. Diagram rules. When defining the causal representations (51) and (52), we performed opposite Keldysh rotations for the dot and the reservoirs. This is motivated by the form of the tunneling Liouvillian Eq. (38), which in the causal representation can be written compactly as

$$
L^{V}=\bar{G}_{1} \bar{J}_{\overline{1}}+\tilde{G}_{1} \tilde{J}_{\overline{1}}=\sum_{q= \pm} G_{1}^{q} J_{\overline{1}}^{q} .
$$

We note the convenient absence of a minus sign on the right-hand side: we can treat the dot superoperators $G_{i}^{q_{i}}$ as if their commute (rather than anticommute) with the reservoir superoperators $J_{k}^{q_{k}}$ as mentioned in the remark after Eq. (20). We can now integrate out the reservoir degrees of freedom in each term of the expansion (34) in the way discussed in Ref. 26 by commuting all reservoir superoperators to the right side using the relation

$$
J_{1}^{q} L^{\mathrm{R}}=\left(L^{\mathrm{R}}-\eta_{1}\left(\omega_{1}+\mu_{1}\right)\right) J_{1}^{q} .
$$

The $m$ th-order contributions to $\Sigma(z)$ then read as

$$
\begin{gathered}
\frac{1}{z-L} G_{1}^{q_{1}} \frac{1}{z-X_{1}-L} G_{2}^{q_{2}} \frac{1}{z-X_{2}-L} \ldots, \\
\frac{1}{z-X_{m-1}} G_{m}^{q_{m}} \frac{1}{z-L} \rho\left(t_{0}\right) \operatorname{Tr}_{\mathrm{R}}\left(J_{\overline{1}}^{q_{1}} J_{\overline{2}}^{q_{2}} \ldots J_{\bar{m}}^{q_{m}} \rho^{\mathrm{R}}\right) .
\end{gathered}
$$

Here, $X_{i}=\sum_{k \leqslant i} x_{k}$, where the summation runs over the reservoir frequencies $x_{k}=\eta_{k}\left(\omega_{k}+\mu_{k}\right)$ of the $G_{k}^{q_{k}}\left(J_{\bar{k}}^{q_{k}}\right)$ originally standing to the left of resolvent $i$. Applying the Wick theorem (60), we can represent the terms diagrammatically by propagator lines connecting vertices that are contracted in pairs by lines with frequencies $x_{k}$. The irreducible parts of these diagrams, i.e., those parts which can not be cut without hitting at least one reservoir contraction, are collected into the irreducible kernel or self-energy superoperator $\Sigma(z)$. In Fig. 1, we show the diagrams for $\Sigma(z)$ to one- and two-loop order. Leaving implicit the sum over all indices, all possible configurations of pair contractions and all orders $m$, we can

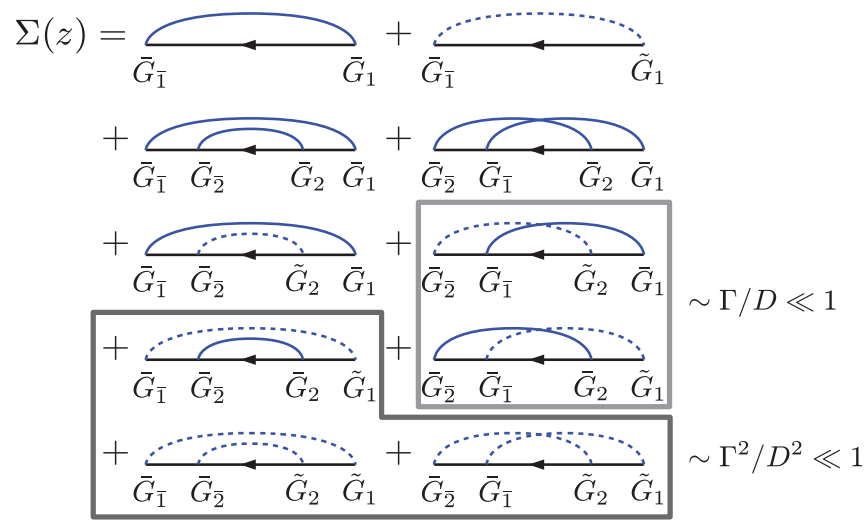

FIG. 1. (Color online) One- and two-loop contributions to the self-energy kernel $\Sigma$. The full black line denotes the free dot propagation, the arrow indicates the ordering of superoperators ("late goes left") in Laplace space that is "inherited" from the time ordering. The curves denote the contraction of dot superoperators. Here, the line connects the dot superoperators whose corresponding reservoir superoperators are contracted: $\langle G J \ldots G J\rangle^{\mathrm{R}}=\langle J J\rangle^{\mathrm{R}} G \ldots G \longrightarrow$ $G \ldots G$. This is in contrast to the standard technique, where a contraction line connects contracted operators themselves. The full blue line denotes a Keldysh contraction $\bar{\gamma}$ of two $\bar{G}$-type vertices [cf. Eq. (64)], whereas the dashed blue contraction corresponds to the retarded contraction $\tilde{\gamma}$ of a $\bar{G}$ with an earlier $\tilde{G}$ vertex to the right [cf. Eq. (63)]. Diagrams with vanishing contractions, i.e., where $\tilde{G}$ is contracted to the right [cf. Eq. (62)], are not drawn. Furthermore, the two-loop diagrams in the light and dark gray boxes contain a $\tilde{\gamma}$ contraction enclosing $k=1$ and, respectively, $k=2$ other vertices and therefore scale with $\Gamma^{k} / D^{k}$ and can be neglected in the wide-band limit. In the causal representation, only 5 diagrams remain, in contrast to formulations using "naive" superoperators (Ref. 30) where 20 terms remain, which partially cancel out. 
write

$$
\begin{aligned}
\Sigma(z)= & (-1)^{P}\left(\prod \gamma_{i j}\right)_{\text {irr }} G_{1}^{-} \frac{1}{z-X_{1}-L} G_{2}^{q_{2}} \cdots \\
& \times G_{m-1}^{q_{m-1}} \frac{1}{z-X_{m-1}-L} G_{m}^{q_{m}} .
\end{aligned}
$$

Here, $\gamma$ denotes that the function $\bar{\gamma}(\tilde{\gamma})$, given by Eq. (64) [Eq. (63)], should be written for pair contraction connecting a $\bar{G}_{i}$ vertex on the left with a $\bar{G}_{j}\left(\tilde{G}_{j}\right)$ vertex on the right. By Eq. (65) it is the earliest vertex (rightmost) that decides the type of contraction, i.e., its indices appear as the argument of the contraction function. Here, $X_{i}$ is now the sum over the frequencies of all reservoir contractions which go over $i$ th resolvent (since contractions that start and end to the left or right cancel out).

Importantly, since on the left we always have $\bar{G}$ (i.e., $G^{q_{1}}$ with $q_{1}=-$ ), the property (55) of the causal field of type $\bar{G}$ is seen to guarantee the conservation of probability:

$$
\operatorname{Tr}_{D} \Sigma(z)=0
$$

Another general property restricts the frequency dependence

$$
K \Sigma(z) K=-\Sigma\left(-z^{*}\right) .
$$

Here, $K=K^{-1}$ is the antilinear superoperator that effects the Hermitian conjugation of an operator (see Appendix G). (Such a transformation of an arbitrary superoperator $S \rightarrow$ $K S K:=S^{c}$ was referred to as "c conjugation" in Ref. 26.) This guarantees through Eqs. (35) and (36) that the reduced density operator remains Hermitian during the time evolution, i.e., $\rho(z)=\rho\left(-z^{*}\right)$. The property (70) derives from the conjugation properties (119) below and from $K L K^{-1}=-L$. The causal representation (68) of the diagrammatic perturbation theory is very useful in general and has been extended to time-dependent problems in Refs. 38 and 50. We now discuss the main advantages that will be important for setting up the RG and the construction of a convenient supervector basis in Sec. II C.

b. Wide-band limit. First of all, the number of contributing terms in Eq. (68) is strongly reduced since the trivial $(\tilde{\gamma})$ and nontrivial $(\bar{\gamma})$ energy dependencies of the contractions are automatically separated, which in other representations has to be done separately. ${ }^{30}$ Here, terms that do not contribute in the wide-bandwidth limit can be identified using the diagram topology as illustrated in Fig. 1. All diagrams where one or more vertices are enclosed between contracted vertices $\bar{G}_{1}$ and $\tilde{G}_{\overline{1}}$ give contributions of order $\Gamma / D \ll 1$ or smaller and can be neglected. This can be proved by careful examination of the poles appearing when closing all integrals in the complex upper half plane (see Ref. 38) or argued in the time representation. ${ }^{51}$ As a result, $\tilde{\gamma}$ contractions can only occur inside $\bar{\gamma}$ contractions in diagrams with more than one loop (see Fig. 1). This results in an exponential reduction in the number of contributing terms.

This feature also naturally suggests a starting point for a two-stage RG approach. First, all terms that contain retarded contractions $\tilde{\gamma}$ can be integrated out explicitly by a one-step diagram resummation, leading to a renormalization of $L$ discussed in Sec. II B4. The remaining diagrams will then contain only $\bar{G}$ superoperators with nontrivial contractions $\bar{\gamma}$, which can be eliminated by a second, continuous RG. This approach will be worked out in detail in Sec. III.

c. Infinite-temperature limit. The choice of the supervector basis is simplified very much by noting that the superoperator structure of $\Sigma(z)$ is strongly restricted in the causal representation: in the wide-band limit (see above), there is only one diagram that starts with $\tilde{G}$ on the right and ends with $\bar{G}$ on the left. We denote this special one-loop diagram by $\tilde{\Sigma}$. Importantly, all other diagrams start and end with a $\bar{G}$ vertex. The $\omega$ integral for the $\tilde{\Sigma}$ diagram can be performed by closing the integration contour in the upper/lower half-plane of the complex plane for $\eta=\mp$ and neglecting small corrections of order $\Gamma / D \ll 1$ :

$$
\tilde{\Sigma}(z)=\int d \omega \bar{G}_{1} \frac{\tilde{\gamma}(\eta \omega)}{z-L-\eta \omega-\eta \mu_{1}} \tilde{G}_{\overline{1}}=-i \frac{\Gamma_{1}}{2} \bar{G}_{1} \tilde{G}_{\overline{1}},
$$

where $\Gamma_{1}=\Gamma_{r}$ for multi-index $1=\eta, \sigma, r, \omega$. The self-energy $\tilde{\Sigma}$ has a clear physical meaning: it is the self-energy one obtains in the infinite-temperature limit. This follows from its definition since all contractions $\bar{\gamma}=0$ for $T=\infty$, and $\tilde{\Sigma}$ is the only diagram in the wide-band limit without this contraction function. As discussed in Sec. II B2, the retarded correlation function of the reservoirs $\tilde{\gamma}$ contains only spectral information [see the discussion of Eq. (63)] and is therefore independent of $T$. Therefore, $\Sigma=\tilde{\Sigma}$ at $T=\infty$. Since at $T=\infty$ no energy scale matters any more, $\tilde{\Sigma}$ is independent of the QD frequency $z$ or any QD energy scale in the problem as well as the cutoff $D$. The dependence on $\Gamma$ remains, however: in the high-temperature limit, all quantum dot states are equally accessible by tunneling processes. This is described by the self-energy $\tilde{\Sigma}$.

The action of $\tilde{\Sigma}$ is very different from that of the Liouvillian $L$ of the isolated QD: it is not super-Hermitian, as $L$ is, but rather anti-super-Hermitian,

$$
\tilde{\Sigma}^{\dagger}=-\tilde{\Sigma},
$$

which simply follows from the Hermitian conjugation property of the causal field superoperators [Eq. (57)]. For $T=\infty$, the effective Liouvillian thus reduces to $L(z)=L+\tilde{\Sigma}(z)$ with stationary state

$$
\rho=\frac{1}{4} \mathbb{1}
$$

which is the maximal entropy state. This follows directly from the causal representation of $\tilde{\Sigma}$ [Eq. (71)], which shows that $\tilde{\Sigma}$ and $\tilde{G}$ share the same right eigenvectors, combined with the probability conservation Eq. (58). By the same argument, it is clear that in this limit the current vanishes: anticipating the result (183) of Sec. II E, we find for the self-energy required for the current equals

$$
\tilde{\Sigma}^{r}(z)=-\left.i \frac{\Gamma_{1}}{2} \bar{G}_{1} \tilde{G}_{\overline{1}}\right|_{r_{1}=r},
$$

where in the sum over 1 we exclude the reservoir index $r$. This also vanishes by Eq. (58) and therefore $\left\langle I^{r}\right\rangle=0$.

d. Cutoff dependence and complete basis. Another advantage of the causal representation is that the cutoff dependence of integrals in the individual diagrams that do contribute in the wide-band limit can be analyzed on the level of superoperators. 
These self-energy contributions seem to depend on the energy integral cutoff $D$. However, using the causal structure of the perturbation theory, one can explicitly see that such dependence cancels out due to the superoperator structure of the vertices (i.e., the matrices multiplying the $D$-dependent contributions to the integrals vanish). The condition for this is that one keeps the complete basis of many-body eigenstates of the dot Hamiltonian. To see this, however, one needs to consider the entire Liouville space, including all off-diagonal density operator elements and not restrict the analysis to only diagonal density matrix elements based on symmetry properties as is often done. The idea is best illustrated by considering the one-loop contributions in Fig. 1. By Eq. (71), the one-loop diagram with a $\tilde{\gamma}$ contraction is explicitly independent of $D$. For the $\bar{\gamma}$ contraction of two $\bar{G}$-type vertices,

$$
\int d \omega_{1} \bar{G}_{1} \frac{\bar{\gamma}_{\overline{1} 1}\left(\eta_{1} \omega_{1}\right)}{z-L-\eta_{1} \omega_{1}-\eta_{1} \mu_{1}} \bar{G}_{\overline{1}},
$$

the $D$ dependence enters through the most divergent part of the integral, obtained by neglecting $L_{D}$ in the denominator. This part is thus proportional to a superoperator that is identically zero due to anticommutation relations (53):

$$
\bar{G}_{1} \bar{G}_{\overline{1}}=-\bar{G}_{\overline{1}} \bar{G}_{1}=\bar{G}_{2} \bar{G}_{\overline{2}}=0 .
$$

Here, we renamed the dummy summation indices $1=$ $\eta_{1}, \sigma_{1}, r_{1}, \omega_{1}$; in fact, only the implicit summation over $\eta_{1}$ is relevant here. Importantly, this argument breaks down as soon as many-body states have been excluded from the Hilbert space basis, e.g., based on their large energy. For example, in the limit $U \rightarrow \infty$, one can exclude the doubly occupied QD state $|2\rangle$ and thereby eliminate Liouville space elements with eigenvalues exceeding $D$. This simplifies the calculations, but for vertex operators projected onto this subspace the relation (76) does not hold anymore (since it is a nonlinear relation). As a result, the explicit $D$ dependence remains and does not cancel out from such expressions, and the cutoff should be set to $D \sim U$ if one makes this approximation.

The above analysis can be extended to higher-order diagrams, and one finds that also there the $D$ dependence drops out. Here, one uses that diagrams containing $\tilde{G}$ vertices are always expressible in terms of the cutoff-independent $\tilde{\Sigma}$ skeleton (see also Sec. II B4). This analysis confirms the observation, made previously after explicit calculations of the kernel $\Sigma(i 0)$, e.g., in Ref. 52, that the cutoff $D$ drops out. Here, however, the cutoff dependence can be completely assessed on a very general level, based on the fermionic superoperator algebra, without any need for the explicit calculation of matrix elements.

\section{Finite-temperature perturbation theory: Elimination of infinite-temperature contractions}

As already mentioned at the end of Sec. II B3, the causal structure of the perturbation theory naturally suggests to proceed in two stages. We first eliminate all diagrams containing the retarded contraction $\tilde{\gamma}$, i.e., the skeleton diagram (71). As discussed in Sec. II B2, the function $\tilde{\gamma}$, given by Eq. (63), only contains spectral information about the reservoirs. Since this contraction always occurs isolated and inside other Keldysh contractions (cf. Sec. II B3), we can use (71) as a skeleton

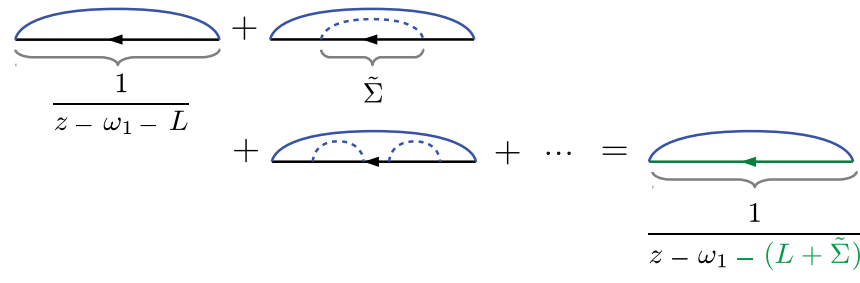

FIG. 2. (Color online) Example resummation of diagrams with a single $\bar{\gamma}$ loop (solid blue curve) and $n=0,1,2, \ldots$ skeleton diagrams $\tilde{\Sigma}$, resulting in one-loop diagram with a renormalized propagator. Since in the wide-band limit $\tilde{\gamma}$ contractions (dashed blue curve) can not contain other vertices, this can be extended to any irreducible diagrams with any number of $\bar{\gamma}$ loops.

diagram and on each propagator line resum the series,

$$
\frac{1}{z-L-X} \sum_{n=0}^{\infty}\left(\tilde{\Sigma} \frac{1}{z-L-X}\right)^{n}=\frac{1}{z-\bar{L}-X},
$$

thereby renormalizing the dot Liouvillian to

$$
\bar{L}=L+\tilde{\Sigma} .
$$

This is illustrated in Fig. 2. As discussed in Sec. II B3 c, $\tilde{\Sigma}$ equals the QD self-energy in the limit of infinite temperature in all reservoirs. The lack of energy dependence of $\tilde{\Sigma}$ reflects that in the high-temperature limit, all QD states are equally accessible via transitions induced by the electrodes. Physically, one expects that the Keldysh contractions $\bar{\gamma}$, describing the nontrivial, temperature-dependent part of the distribution function, ${ }^{53}$ will drop out in this limit.

In a second stage, we calculate the finite-temperature effects, which are all incorporated through the self-energy $\bar{\Sigma}(z)$. Its diagrammatic perturbation series has the same structure as for $\Sigma$, but is expressed entirely in terms of the contraction $\bar{\gamma}$, the vertex $\bar{G}$, and the Liouvillian $\bar{L}$ :

$$
\begin{aligned}
\bar{\Sigma}(z)= & (-1)^{P}\left(\prod \bar{\gamma}_{i j}\right)_{\mathrm{irr}} \\
& \times \bar{G}_{1} \frac{1}{z-X_{1}-\bar{L}} \bar{G}_{2} \ldots \frac{1}{z-X_{n-1}-\bar{L}} \bar{G}_{n} .
\end{aligned}
$$

Summing this renormalized perturbation theory, one obtains, of course, the same exact result (36) for the effective Liouvillian, which, however, is now decomposed in a different way:

$$
L(z)=\bar{L}+\bar{\Sigma}(z) .
$$

All contractions $\tilde{\gamma}$ have been eliminated simultaneously in the above transformation of the perturbation series. This was previously referred to as the discrete step of the real-time renormalization group procedure. ${ }^{26}$ In the Anderson model in the wide-band limit, only one skeleton diagram contributes to $\bar{L}$ through $\tilde{\Sigma}$ and no renormalization of vertex $\bar{G}$ is required to eliminate $\tilde{\gamma} \cdot{ }^{54}$ This discrete first step is a necessary preparation for the second-step RG in which we will integrate out the $\bar{\gamma}$ contractions as well in a continuous RG flow. In contrast to the $\bar{G}$, the vertices $\tilde{G}$ do not share the left zero eigensupervector $\left(Z_{L} \mid\right.$ with the effective Liouvillian [cf. Eq. (86)]. If they were still present during a process of continuous renormalization, this would lead to divergences whenever the zero eigenvalue of $L$ would appear in the resolvent $(z-X-L)^{-1}$ (see also Ref. 55). The vertices $\tilde{G}$ must therefore be integrated out before 
any continuous RG can be formulated. This is a characteristic feature of an RG for dissipative systems that exhibits a stationary state (a zero eigensupervector). The causal structure of the renormalized perturbation theory (79) makes clear that this elimination has been achieved: the zero eigenvalue term of $\bar{L}$ always drops out due to the presence of $\bar{G}$ 's adjacent to the propagator $(z-X-\bar{L})^{-1}$.

At this point, we can already make an observation that is important for the construction of an explicit expansion for the effective Liouvillian $L(z)$ later on in Sec. II C5: no continuous RG scheme, which reorganizes the perturbation series Eq. (79) involving only $\bar{G}$ vertices, can ever generate terms of the form $\bar{G} \ldots \tilde{G}$, i.e., with a $\tilde{G}$ superoperator standing on the far right. In the notation of Sec. II C5, this implies that the coefficient of the term $\left.\mid Z_{R}\right)\left(Z_{R} \mid\right.$ in the expansion Eq. (136) is not changed during a $\mathrm{RG}$ flow [or any nonperturbative approximation to the series (79)].

What was done so far can be understood as a formal expansion around the infinite-temperature limit as a reference point. We start from the exact solution for the infinite-temperature, wide-band limit and then reformulate the perturbation theory for finite temperature (including the zero-temperature limit of interest.) This is not to be confused with the expansion in inverse powers of $T$ : we formally expand in the Keldysh distribution functions (contractions) $\bar{\gamma}$. Actually, one meets this expansion already in most standard applications of the generalized master equation approach where one also calculates the kernel $\Sigma(i 0)$, obtaining "rates" that include Fermi's golden rule: this corresponds to the correction to $\tilde{\Sigma}$ of first order in $\bar{\gamma}$. Here, we reformulated the exact perturbation theory around this reference point in order to perturbatively calculate the important higher-order corrections to that result. To this end, we performed a first natural step by exactly incorporating the "trivial" infinite-temperature fluctuations into a redefinition of the Liouvillian. This first step is the crucial starting point for the RT-RG approach that we will develop to incorporate the "nontrivial" finite-temperature fluctuations also nonperturbatively: it prevents a serious technical problem related to the zero eigenvalue of the stationary nonequilibrium state from appearing (see Secs. III A and II C5).

\section{Basis of the Anderson model in Liouville Fock space}

To obtain explicit RG equations that can be solved numerically in an efficient way, we need to construct a basis that exploits the advantages of (i) selection rules for the self-energy superoperator induced by the global symmetries, (ii) the causal structure of $\bar{\Sigma}(z)$, and (iii) the fermion-parity superselection rule. Again, these can be useful in the perturbation theory as well, so we discuss them here.

\section{Liouville space bra-ket formalism}

By Eqs. (68) and (79), the QD self-energy $\Sigma(\bar{\Sigma})$ is a functional of the QD Liouvillian $L(\bar{L})$ and the vertex superoperators $G^{q}(\bar{G})$, which linearly act on the QD Hilbert space of many-body states. To explicitly calculate these superoperators, it is convenient to introduce a "bra-ket" formalism analogous to that of standard quantum mechanics: we write a 16-component supervector (representing an operator acting on the four-dimensional Hilbert space) as a rounded superket
$A=\mid A)$ and introduce its dual supervector $\left(A \mid \bullet=\operatorname{Tr}\left(A^{\dagger} \bullet\right)\right.$ as a linear functional acting on operators. An operator $A$ is orthogonal to $B$ if $(A \mid B)=\operatorname{Tr}\left(A^{\dagger} B\right)=0$ (see, e.g., Ref. 56). The dual vector may be written as the Hermitian conjugate of a superket:

$$
\left.(A|=| A)^{\dagger}, \quad \mid A\right)=\left(\left.A\right|^{\dagger},\right.
$$

where $\dagger$ is not to be confused with the supervector corresponding to the Hermitian conjugate of the operator $A, A^{\dagger}=\mid A^{\dagger}$ ). An operator basis $\left.\mid A_{i}\right), i=1, \ldots, 16$, of mutually orthonormal operators $\operatorname{Tr}\left(A_{i}^{\dagger} A_{j}\right)=\delta_{i, j}$ is complete if any operator $B$ can be expanded as $B=\sum_{i}\left(A_{i} \mid B\right) A_{i}$ with coefficients $\left(A_{i} \mid B\right)=$ $\operatorname{Tr}\left(A_{i}^{\dagger} B\right)$. Any superoperator $S$ acting on such operators can then be expressed in general as a sum of 256 terms:

$$
\left.S=\sum_{i, j=1}^{16}\left(A_{i}|S| A_{j}\right) \mid A_{i}\right)\left(A_{j} \mid .\right.
$$

\section{Liouville-Fock-space basis}

To maximally reduce the number of terms in the expansion (82) of physical superoperators, we now exploit the close analogy between our Liouville field superoperators in the causal representation and the usual fermionic field operators. In this section, we will first construct a suitable orthonormal basis of operators (supervectors) in which any QD operator can be expanded. In Sec. II C3, we analyze their transformation properties under the symmetry transformations of the Anderson model. In this basis, we can then in Sec. II C5 easily construct superoperator expansions compatible with these symmetries.

We start from the key property (55) of the vertex operators in the causal representation. In bra-ket notation, the trace operation corresponds to the action

$$
\operatorname{Tr}_{D} \bullet=\operatorname{Tr}_{D} \mathbb{1} \bullet=2\left(Z_{L}\right.
$$

of the dual of the normalized supervector

$$
\left.\mid Z_{L}\right)=\frac{1}{2} \mathbb{1} .
$$

Therefore, by Eq. (55), $\left(Z_{L} \mid\right.$ is a left zero eigensupervector of $\bar{G}$, and by (57) it follows that $\left.\mid Z_{L}\right)$ must be a right eigensupervector of $\tilde{G}$ :

$$
\left(Z_{L}\left|\bar{G}_{1}=0, \quad \tilde{G}_{1}\right| Z_{L}\right)=0 .
$$

We can formally consider the state annihilated by operators $\tilde{G}$ as a "supervacuum." (Note that the "supervacuum" state is the most symmetric dot operator similar to standard field theories where the vacuum state usually is the most symmetric one.) This vacuum supervector is proportional to the physical infinite-temperature density operator [cf. Eq. (73)] with maximal von Neumann entropy $S=-\operatorname{Tr}_{D}\{\rho \ln (\rho)\}$. It is then also natural to construct the corresponding right zero eigensupervector of $\bar{G}_{1}$ whose dual is the left zero eigensupervector of $\tilde{G}_{1}$ :

$$
\left.\bar{G}_{1} \mid Z_{R}\right)=0, \quad\left(Z_{R} \mid \tilde{G}_{1}=0 .\right.
$$

Since $\bar{G}_{1}$ is a non-Hermitian superoperator, these eigensupervectors are not simply related by Hermitian conjugation in Liouville space $\left.\mid Z_{R}\right) \neq\left(\left.Z_{L}\right|^{\dagger}\right.$ [cf. Eq. (81)]. 
The operator $Z_{R}$ will be found to play a key role throughout this work. In Appendix E, we discuss its many interesting properties, most prominently, its relation to the fermion parity, its similarity to Grassmann numbers, and its relation to the spin and charge rotations of Sec. II C3. To construct $\left.\mid Z_{R}\right)$, we now exploit the close analogy to the usual field operators: $\bar{G}_{1}$ is a creation operator in Liouville Fock space since its Hermitian superconjugate $\tilde{G}_{\overline{1}}$ annihilates the supervacuum state $\mid Z_{L}$ ) by Eq. (85). The state annihilated by $\bar{G}_{1}$ is therefore simply the maximally occupied state in the QD Liouville Fock space, starting from the vacuum ${ }^{57}$

$$
\left.\left.\mid Z_{R}\right)=\prod_{\sigma}\left(\prod_{\eta} \bar{G}_{\eta \sigma}\right) \mid Z_{L}\right) .
$$

As for the usual fermionic field operators, the action of creation operators preserves the normalization: this follows directly from Eq. (81) and the anticommutation relations (53):

$$
\left(Z_{R} \mid Z_{R}\right)=\left(Z_{L}\left|\prod_{\sigma}\left(\prod_{\eta} \tilde{G}_{\bar{\eta} \sigma} \bar{G}_{\eta \sigma}\right)\right| Z_{L}\right)=\left(Z_{L} \mid Z_{L}\right)=1 .
$$

Note that by Eq. (53), reordering the $\bar{G}$ 's in the definition of $Z_{R}$ only amounts to an unimportant redefinition of the overall sign. Using Eq. (51), we can write for the explicit action on any operator (denoted by $\bullet$ ) with even fermion parity

$$
\bar{G}_{+\sigma} \bar{G}_{-\sigma} \bullet=\frac{1}{2}\left(\left[n_{\sigma}, \bullet\right]_{+}-1+d_{\sigma}^{\dagger} \bullet d_{\sigma}-d_{\sigma} \bullet d_{\sigma}^{\dagger}\right) \text {. }
$$

Inserting this into Eq. (87), we obtain

$$
\left.\mid Z_{R}\right)=\frac{1}{2} \prod_{\sigma}\left(2 n_{\sigma}-\mathbb{1}\right) .
$$

Since $\left(Z_{L} \mid\right.$ is a left eigenvector of $\bar{G}$ [cf. Eq. (85)], we immediately see that the two zero eigensupervectors are orthonormal, $\left(Z_{L} \mid Z_{R}\right)=0$. Therefore, it is natural to include $\left.\mid Z_{R}\right)$ into the orthonormal Liouville space basis. By successively acting on the supervacuum state $\left.\mid Z_{L}\right)$, we can generate more normalized, orthogonal operators. There are in total eight bosonic operators:

$$
\begin{gathered}
\left.\mid Z_{L}\right)=\frac{1}{2} \mathbb{1}, \\
\left.\left.\mid \chi_{\sigma}\right)=\bar{G}_{+\sigma} \bar{G}_{-\sigma} \mid Z_{L}\right), \\
\left.\left.\mid T_{\eta}\right)=\eta \bar{G}_{\eta \uparrow} \bar{G}_{\eta \downarrow} \mid Z_{L}\right), \\
\left.\left.\mid S_{\sigma}\right)=\bar{G}_{+\sigma} \bar{G}_{-\bar{\sigma}} \mid Z_{L}\right), \\
\left.\left.\mid Z_{R}\right)=\bar{G}_{+\uparrow} \bar{G}_{-\uparrow} \bar{G}_{+\downarrow} \bar{G}_{-\downarrow} \mid Z_{L}\right),
\end{gathered}
$$

and there are eight fermionic operators:

$$
\begin{aligned}
& \left.\left.\mid \alpha_{+, \sigma}^{+}\right)=\bar{G}_{+\sigma} \mid Z_{L}\right), \\
& \left.\left.\mid \alpha_{-, \sigma}^{+}\right)=\sigma \bar{G}_{-\bar{\sigma}} \mid Z_{L}\right), \\
& \left.\left.\mid \alpha_{+, \sigma}^{-}\right)=\bar{G}_{+\sigma} \bar{G}_{+\bar{\sigma}} \bar{G}_{-\bar{\sigma}} \mid Z_{L}\right), \\
& \left.\left.\mid \alpha_{-, \sigma}^{-}\right)=\sigma \bar{G}_{-\bar{\sigma}} \bar{G}_{-\sigma} \bar{G}_{+\sigma} \mid Z_{L}\right) .
\end{aligned}
$$

The labeling of these basis supervectors is motivated by their explicit expressions in terms of the field operators $d_{\sigma}, d_{\sigma}^{\dagger}$ [see Eqs. (103)-(114)] and their behavior under symmetry transformations, which will be discussed in the next section. We see that the index $v$ of the operators $\left.\mid \alpha_{\eta \sigma}^{v}\right)$ only has a meaning in Liouville space: it distinguishes $v=+$ states with one excess excitation relative to the supervacuum $\mid Z_{L}$ ) (superparticles) from $v=-$ states with one deficit particle with respect to the maximally occupied superstate $\left.\mid Z_{R}\right)$ (superholes). By construction, these 16 operators form a complete orthonormal basis of the QD Liouville space. This basis includes only one operator that has nonzero trace, namely, $\left.\mid Z_{L}\right)=\frac{1}{2} \mathbb{1}$. Since all other supervectors are orthogonal to $\left.\mid Z_{L}\right)$, their corresponding operators are traceless according to Eq. (83).

We emphasize that the choice of basis supervectors $\left.\mid Z_{L}\right)$ and $\left.\mid Z_{R}\right)$ relies on two general physical properties of the problem: the probability conservation [cf. Eqs. (69) and (85)] and the fermion parity (cf. Appendix E). The choice of the signs of the remaining operators, however, is motivated by considering the symmetry transformations of the Anderson model. These will be discussed in the next section. The related approach of Prosen was also introduced by an explicit construction of the Liouville Fock space ${ }^{31}$ (see Appendix C).

Before we proceed, we emphasize the necessity of working with a complete basis for the QD Liouville space, which includes operators that are nondiagonal in both spin and/or charge quantum numbers. In perturbation theory, one can disregard all matrix elements of the self-energy $\Sigma(z)$ involving the latter operators (cf. Sec. II B3) due to the conservation laws (101). However, the states propagating in the inner part of a diagram contributing to $\Sigma(z)$ (i.e., a virtual intermediate state) are less restricted by the conservation laws, requiring the matrix elements of the vertices and the QD Liouvillian $L$ between all the off-diagonal operators. ${ }^{58}$ In the RT-RG that we set up below, such matrix elements involving oddfermion-parity operators can not be avoided for $\Sigma(z)$ as well since one needs to describe the renormalization of all virtual intermediate states, both fermionic as well as bosonic ones.

\section{Irreducible transformation under symmetry operations}

For the total system of QD and reservoirs, both the charge and spin components along the magnetic field are conserved [cf. Eqs. (25) and (26)]. The particle-number conservation on the dot is conveniently expressed using the charge-polarization operator

$$
T_{z}=\frac{1}{2}(n-1),
$$

which measures the difference of the occupation probabilities of the empty and doubly occupied QD states $|0\rangle$ and $|2\rangle$. It follows from Eqs. (25) and (26) that the dot superoperators for charge polarization [cf. Eq. (43)] and spin

$$
\begin{gathered}
L^{T_{z}}=\left[T_{z}, \bullet\right]_{-}=\frac{1}{2} L^{n}, \\
L^{S_{z}}=\left[S_{z}, \bullet\right]_{-},
\end{gathered}
$$

respectively, commute with each other as well as with the Liouvillian $L$ and the self-energy $\Sigma(z)$. Therefore, the effective Liouvillian $L(z)=L+\Sigma(z)$, which determines the time evolution of the reduced density operator, conserves these superobservables (see Appendix F for a derivation):

$$
\begin{aligned}
& {\left[L(z), L^{T_{z}}\right]_{-}=0,} \\
& {\left[L(z), L^{S_{z}}\right]_{-}=0 .}
\end{aligned}
$$


Thus, $L(z)$ can be simultaneously block diagonalized with the superoperators for charge and spin polarization and they have common eigensupervectors. In the basis of Sec. II C3, the initial dot Hamiltonian operator Eq. (1) has the form

$$
\begin{aligned}
H= & \left.\left.\left(2 \epsilon+\frac{U}{2}\right) \mid Z_{L}\right)+\frac{U}{2} \mid Z_{R}\right) \\
& \left.\left.+2\left(\epsilon+\frac{U}{2}\right) \mid T_{z}\right)+B \mid S_{z}\right) .
\end{aligned}
$$

In two special cases, the total system has a higher symmetry: for $\epsilon=-U / 2$ and $\mu_{L}=\mu_{R}$, it exhibits full charge-rotation symmetry, whereas for $B=0$ it has full spin-rotation symmetry. These full symmetry groups are obtained by adding the operators $S_{x}$ and $S_{y}$ to $S_{z}$ [cf. Eq. (107)], giving the SU(2) spin algebra, and by adding $T_{x}$ and $T_{y}$ to $T_{z}$ [cf. Eq. (111)], giving another SU(2) algebra generating "charge rotations" (see the following). The construction of superoperators that transform in the simplest possible way under these symmetry operations is greatly simplified by first constructing basis operators that can be classified with respect to the corresponding irreducible representations. Importantly, the Liouville Fock basis (91)(96) that we constructed using the causal field superoperators is already very close to a symmetry-adapted basis and we merely need to complete the classification. We first group these operators according to their even or odd fermion parity, and refer to these as bosonic and fermionic operators, respectively. We subsequently classify them according to the transformation behavior under the two SU(2) rotation groups as an irreducible tensor operator (ITO). This allows us to identify supervectors corresponding to Hilbert-space operators that are diagonal with respect to the QD charge and/or the QD spin. In perturbation theory, one only needs the self-energies connecting such diagonal components of the density matrix. In the RG that we set up below, this is no longer true. Still, it is important to single out this block of matrix elements of $\Sigma(z)$, $\bar{\Sigma}(z)$, and $L(z)$.

One-half of the Liouville space is then spanned by eight bosonic operators with integer charge- and spin-ITO ranks:

(i) The zero eigenvectors of the vertex superoperators are scalars (rank-0 spin and charge ITOs) with respect to both spin and charge rotations since the zero-eigenvalue equations (85) and (86) are invariant under these transformations. They are therefore charge- and spin-diagonal operators. This is also clear from their explicit form in terms of the Casimir operators of the spin- $\left(S^{2}=\sum_{i} S_{i}^{2}\right)$ and charge-rotation $\left(T^{2}=\sum_{i} T_{i}^{2}\right)$ $\mathrm{SU}(2)$ Lie algebras:

$$
\begin{gathered}
\left.\mid Z_{L}\right)=\frac{1}{2} \mathbb{1}=\frac{4}{3}\left(T^{2}+S^{2}\right), \\
\left.\mid Z_{R}\right)=2 n_{\uparrow} n_{\downarrow}-n+\frac{1}{2} \mathbb{1}=\frac{2}{3}\left(T^{2}-S^{2}\right) .
\end{gathered}
$$

(ii) The generators of rotations in spin space are

$$
\begin{gathered}
\left.\left.\mid S_{0}\right)=\frac{1}{\sqrt{2}} \sum_{\sigma} \sigma \mid \chi_{\sigma}\right)=\frac{1}{\sqrt{2}} \sum_{\sigma} \sigma n_{\sigma}, \\
\left.\mid S_{\sigma}\right)=d_{\sigma}^{\dagger} d_{\bar{\sigma}}, \quad \sigma=\uparrow, \downarrow= \pm .
\end{gathered}
$$

The operators $\left.\left.\left.-\mid S_{+}\right), \mid S_{0}\right), \mid S_{-}\right)$transform as the components of a rank-1 spin ITO (vector), i.e., simpler than the more familiar Cartesian components of the spin operator

$$
\left.\left.\left.\left.\left.\mid S_{x, y}\right)=\frac{1}{2(i)}\left(\mid S_{+}\right) \pm \mid S_{-}\right)\right), \quad \mid S_{z}\right)=\frac{1}{\sqrt{2}} \mid S_{0}\right),
$$

which satisfy $\left.\left.\left.\left[\mid S_{i}\right), \mid S_{j}\right)\right]_{-}=i \epsilon_{i j k} \mid S_{k}\right)$. They transform as a rank-0 ITO (scalar) with respect to charge rotations and are therefore charge diagonal.

(iii) The generators of rotations in charge space are

$$
\begin{gathered}
\left.\left.\mid T_{0}\right)=\frac{1}{\sqrt{2}} \sum_{\sigma} \mid \chi_{\sigma}\right)=\frac{1}{\sqrt{2}}(n-1), \\
\left.\mid T_{+}\right)=d_{\uparrow}^{\dagger} d_{\downarrow}^{\dagger}, \\
\left.\mid T_{-}\right)=d_{\downarrow} d_{\uparrow} .
\end{gathered}
$$

The operators $\left.\left.\left.-\mid T_{+}\right), \mid T_{0}\right), \mid T_{-}\right)$transform as a rank-1 ITO (vector) under rotations in charge space, and as a rank-0 ITO (scalar) with respect to spin rotations. Therefore, they are all spin diagonal. They are more convenient than the Cartesian components

$$
\left.\left.\left.\left.\left.\mid T_{x, y}\right)=\frac{1}{2(i)}\left(\mid T_{+}\right) \pm \mid T_{-}\right)\right), \quad \mid T_{z}\right)=\frac{1}{\sqrt{2}} \mid T_{0}\right)
$$

satisfying the $\mathrm{SU}(2)$ algebra $\left.\left.\left.\left[\mid T_{i}\right), \mid T_{j}\right)\right]_{-}=i \epsilon_{i j k} \mid T_{k}\right)$. In contrast to the spin operators, here the indices $x, y, z$ are not related to the axes in the real space, but merely label the components of the $\mathrm{SU}(2)$ generators.

In the bosonic sector, we can thus use either

$$
\left.\left.\left.\mid \chi_{\sigma}\right)=n_{\sigma}-\frac{1}{2} \mathbb{1}=\frac{1}{\sqrt{2}}\left[\mid T_{0}\right)+\sigma \mid S_{0}\right)\right],
$$

or $\left.\mid S_{0}\right)$ and $\left.\mid T_{0}\right)$ as basis vectors. Only the latter two are adapted to charge- and spin-rotation symmetry, but the former two allow for greater notational simplicity. Both will be used.

The other half of the QD Liouville space is spanned by eight more fermionic operators with half-integer charge- and spin-ITO ranks:

(i) The fermionic operators, denoted by $\alpha_{\eta, \sigma}^{\nu}$, act in three subspaces of dimension 2 labeled by $\sigma= \pm$ (spin), $\eta= \pm$ (particle hole), and, additionally, $v= \pm$. These basis operators are ITOs of rank $\frac{1}{2}$ with respect to both charge $(\eta)$ and spin rotations $(\sigma)$. For $v=+$ these are the rank- $\frac{1}{2}$ spin ITOs constructed from creation and annihilation operators:

$$
\begin{array}{cl}
\left.\left.\mid \alpha_{+, \sigma}^{+}\right)=\frac{1}{\sqrt{2}} d_{\sigma}^{\dagger}, \quad \mid \alpha_{-, \sigma}^{+}\right)=\frac{1}{\sqrt{2}} \sigma d_{\bar{\sigma}}, \\
\left.\left.\left.\left.\mid \alpha_{+, \sigma}^{-}\right)=2 \mid Z_{R} \alpha_{+\sigma}^{+}\right), \quad \mid \alpha_{-, \sigma}^{-}\right)=2 \mid Z_{R} \alpha_{-\sigma}^{+}\right) .
\end{array}
$$

All these operators are both charge and spin off diagonal. The above explicit form emphasizes that there is an additional set of fermionic operators in the charge off-diagonal subspace that is linearly independent of the standard field operators $d_{\sigma}$ and $d_{\sigma}^{\dagger}$ [see the discussion of the index $v$ after Eq. (96)]. Noting the property $\left(2 Z_{R}\right)^{2}=\mathbb{1}$, we find the following explicit relation between these two sets: with $\bar{v}=-v$,

$$
\left.\left.\mid \alpha_{\eta, \sigma}^{\nu}\right)=2 Z_{R} \mid \alpha_{\eta, \sigma}^{\bar{\nu}}\right) \text {. }
$$

The above simple transformation behavior of the basis supervectors motivates all the relative signs that we anticipated in writing Eqs. (91)-(96). The basis is therefore completely fixed up to irrelevant phases by general physical 
TABLE I. Fermion-parity and irreducible transformation behavior of basis operators under spin and charge rotations. Schematically denoting these operators by $\left.\mid s, m_{s} ; t, m_{t}\right)$, these transform as (i) spin-irreducible tensor operators ( $S$-ITOs) with rank $s$ and index $\left.\left.m_{s}, L^{S_{z}} \mid s, m_{s} ; t, m_{t}\right)=m_{s} \mid s, m_{s} ; t, m_{t}\right)$ and $\left.L^{S_{ \pm}} \mid s, m_{s} ; t, m_{t}\right)=$ $\left.\sqrt{s(s+1)-m_{s}\left(m_{s} \pm 1\right)} \mid s, m_{s} \pm 1 ; t, m_{t}\right)$, and (ii) as charge ITOs ( $T$-ITOs) with rank $t$ and index $\left.m_{t}, L^{T_{z}} \mid s, m_{s} ; t, m_{t}\right)=m_{s} \mid s, m_{s} ; t, m_{t}$ ) and $\left.\left.L^{T_{ \pm}} \mid s, m_{s} ; t, m_{t}\right)=\sqrt{t(t+1)-m_{t}\left(m_{t} \pm 1\right)} \mid s, m_{s} ; t, m_{t} \pm 1\right)$. All zero-index $S$-ITOs ( $T$-ITOs) correspond to operators acting on Hilbert space that are diagonal in spin (charge).

\begin{tabular}{lcccc}
\hline \hline Operator & $\begin{array}{c}\text { Fermion } \\
\text { parity }\end{array}$ & $\begin{array}{c}S \text {-ITO } \\
(\text { rank,index) }\end{array}$ & $\begin{array}{c}T \text {-ITO } \\
(\text { rank, index) }\end{array}$ & \\
\hline $\left.\mid Z_{i}\right)$ & + & $(0,0)$ & $(0,0)$ & $i=L, R$ \\
$\left.\mid S_{m}\right)$ & + & $(1, m)$ & $(0,0)$ & $m=0, \pm 1$ \\
$\left.\mid T_{m}\right)$ & + & $(0,0)$ & $(1, m)$ & $m=0, \pm 1$ \\
$\left.\mid \alpha_{\eta, \sigma}^{v}\right)$ & - & $\left(\frac{1}{2}, \frac{\sigma}{2}\right)$ & $\left(\frac{1}{2}, \frac{\eta}{2}\right)$ & $\eta, \sigma= \pm 1$ \\
\hline \hline
\end{tabular}

properties (probability conservation, symmetries) together with the supervector normalization. Table I summarizes the transformation properties.

Finally, we note the transformation behavior under Hilbertspace Hermitian conjugation, corresponding to an antilinear superoperator $K$ [see Eq. (70)]. For all diagonal basis operators $D=Z_{L}, Z_{R}, \chi_{\sigma}, S_{0}, T_{0}$,

$$
K \mid D)=\mid D),
$$

whereas for the off-diagonal operators

$$
\left.\left.\left.\left.\left.\left.K \mid S_{ \pm}\right)=\mid S_{\mp}\right), \quad K \mid T_{ \pm}\right)=\mid T_{\mp}\right), \quad K \mid \alpha_{\eta, \sigma}^{\nu}\right)=-v \eta \sigma \mid \alpha_{\bar{\eta}, \bar{\sigma}}^{\nu}\right) .
$$

\section{Expansion of the vertices}

The calculation of the bra-ket representation of the vertex superoperators in the basis equations (91)-(96) reduces entirely to the calculation of

$$
\begin{aligned}
\bar{G}_{+\sigma}= & \left.\mid \alpha_{+, \sigma}^{+}\right)\left(Z_{L}|+\sigma| Z_{R}\right)\left(\alpha_{-, \bar{\sigma}}^{-} \mid\right. \\
& \left.+\sigma \mid T_{+}\right)\left(\alpha_{+, \bar{\sigma}}^{+}|+| \alpha_{-, \sigma}^{-}\right)\left(T_{-}|+| \alpha_{+, \sigma}^{-}\right)\left(\chi_{\bar{\sigma}} \mid\right. \\
& \left.-\sigma \mid \chi_{\sigma}\right)\left(\alpha_{-, \bar{\sigma}}^{+}|+\sigma| S_{\sigma}\right)\left(\alpha_{-, \sigma}^{+}|-| \alpha_{+, \bar{\sigma}}^{-}\right)\left(S_{\bar{\sigma}} \mid .\right.
\end{aligned}
$$

This is easily performed using the second quantization technique that we introduced in Liouville Fock space, i.e., using algebra rather than the explicit matrix representations of Sec. II C3. All other vertices follow from general relations: first, using the transformation under Hermitian conjugation [see Eq. (70)],

$$
\bar{G}_{\eta \sigma}=(-1)^{L^{n}+1} K \bar{G}_{\bar{\eta} \sigma} K,
$$

the result for opposite charge index $\eta=-$ follows from Eqs. (116) and (117):

$$
\begin{aligned}
\bar{G}_{-\sigma}= & \left.-\sigma \mid \alpha_{-, \bar{\sigma}}^{+}\right)\left(Z_{L}|-| Z_{R}\right)\left(\alpha_{+, \sigma}^{-} \mid\right. \\
& \left.-\sigma \mid \alpha_{+, \bar{\sigma}}^{-}\right)\left(T_{+}|-| T_{-}\right)\left(\alpha_{-, \sigma}^{+}|+\sigma| \alpha_{-, \bar{\sigma}}^{-}\right)\left(\chi_{\bar{\sigma}} \mid\right. \\
& \left.-\mid \chi_{\sigma}\right)\left(\alpha_{+, \sigma}^{+}|-| S_{\bar{\sigma}}\right)\left(\alpha_{+, \bar{\sigma}}^{+}|+\sigma| \alpha_{-, \sigma}^{-}\right)\left(S_{\sigma} \mid .\right.
\end{aligned}
$$

The vertex superoperators $\tilde{G}_{\eta \sigma}$ are obtained from $\bar{G}_{\bar{\eta} \sigma}$ by the Hermitian conjugation relation (57) between the field superoperators. In the Liouville bra-ket formalism, this simply means that we can interchange bra and ket vectors in the expansions (118) and (120) to obtain $\tilde{G}_{\mp \sigma}$.

We point out that under the two-loop RG flow to be discussed in Sec. III, the structure of the vertex operators is changed and the above relations cease to hold $[\bar{G}$ will be modified whereas $\tilde{G}$ is not changed, implying that Eq. (57) breaks down]. However, in Sec. IV C, we show how such vertex corrections can be incorporated effectively into the flow of the effective Liouvillian only, allowing us to work with the "bare" vertices having the nice properties discussed above. Two properties of the bare vertices that remain valid under the RG flow are

$$
\begin{aligned}
& \left(\alpha_{\eta \sigma}^{+} \mid \bar{G}_{1} \propto\left(Z_{L} \mid \text { or } 0,\right.\right. \\
& \left.\left.\bar{G}_{1} \mid \alpha_{\eta \sigma}^{-}\right) \propto \mid Z_{R}\right) \text { or } 0 .
\end{aligned}
$$

Equation (121) follows since $\mid \alpha_{\eta \sigma}^{+}$) is obtained from the vacuum $\mid Z_{L}$ ) by the action of a single creation superoperator [cf. Eq. (96)]. Using Eq. (57),

$$
\left(\alpha_{\eta, \sigma}^{+} \mid=\sigma^{(1-\eta) / 2}\left(Z_{L} \mid \tilde{G}_{\bar{\eta},(\eta \sigma)} .\right.\right.
$$

When inserted in the left-hand side of Eq. (121), this $\tilde{G}$ can be anticommuted past the $\bar{G}$ using Eq. (53) and the supervacuum property (85), $\left(Z_{L} \mid \bar{G}=0\right.$. Analogously, Eq. (122) follows by noting that Eq. (96) for the $\left.\mid \alpha_{\eta, \sigma}^{-}\right)$can be rewritten as a single destruction superoperator acting on the maximally occupied state in Liouville Fock space:

$$
\left.\left.\mid \alpha_{\eta, \sigma}^{-}\right)=\bar{\sigma}^{(1-\eta) / 2} \tilde{G}_{\eta,(\eta \sigma)} \mid Z_{R}\right) .
$$

Commuting the $\bar{G}$ to the right and using $\bar{G} \mid Z_{R}$ ) $=0$ [Eq. (86)], on the right of each term we obtain Eq. (122).

\section{Expansion of the effective Liouvillian}

a. Causal structure. By the general properties (85) and (86), the vertices must have an expansion of the form [confirmed by the explicit results (118)-(120)]

$$
\begin{aligned}
\bar{G} & \left.=\cdots \mid Z_{R}\right)(\bullet|+\cdots| \bullet)\left(Z_{L} \mid+\cdots,\right. \\
\tilde{G} & =\cdots \mid \bullet)\left(Z_{R}|+\cdots| Z_{L}\right)(\bullet \mid+\cdots,
\end{aligned}
$$

where the remaining terms involve neither $Z_{L}$ nor $Z_{R}$. Therefore, the terms in the expansion of the effective Liouvillian involving these vectors are strongly restricted. Combined with the general causal structure of the perturbative series, i.e., the way $\bar{G}$ and $\tilde{G}$ can appear, this imposes further constraints (cf. Sec. II B3):

(i) Terms of the form $\left.\mid Z_{L}\right)(\bullet \mid$ are prohibited by probability conservation, since otherwise the trace condition (69) would be violated:

$$
\begin{aligned}
\left(Z_{L} \mid \bar{L}\right. & =\left(Z_{L} \mid L(z)=\left(Z_{L} \mid \Sigma(z)\right.\right. \\
& =\left(Z_{L} \mid \tilde{\Sigma}=\left(Z_{L} \mid \bar{\Sigma}(z)=0 .\right.\right.
\end{aligned}
$$

This is guaranteed by the causal structure, which requires that the leftmost vertex is always of the type $\bar{G}$, with expansion Eq. (125). 
(ii) Terms of form $\mid \bullet)\left(Z_{R} \mid\right.$ can only appear due to the diagrams collected in $\tilde{\Sigma}=-i \frac{1}{2} \Gamma_{1} \bar{G}_{1} \tilde{G}_{\overline{1}}$. Expanding Eq. (71) in the basis (91)-(96), one finds that only the term $\left.\mid Z_{R}\right)\left(Z_{R} \mid\right.$ with coefficient $-i 4 \Gamma$ can appear [see also Eq. (134)]. Importantly, this implies that $\left.\mid Z_{R}\right)$ is a right eigenvector of both $\bar{L}$ as well as the exact effective Liouvillian $L(z)$ and the kernel $\Sigma(z)$ [see the discussion of Eq. (136)]:

$$
\begin{aligned}
\left.\bar{L} \mid Z_{R}\right) & \left.\left.=L(z) \mid Z_{R}\right)=\Sigma(z) \mid Z_{R}\right) \\
& \left.\left.=\tilde{\Sigma} \mid Z_{R}\right)=-4 i \Gamma \mid Z_{R}\right) .
\end{aligned}
$$

In contrast,

$$
\left.\bar{\Sigma}(z) \mid Z_{R}\right)=0
$$

Note that this eigenvector and eigenvalue are independent of the QD frequency $z$.

(iii) The term $\left.\mid Z_{R}\right)\left(Z_{L} \mid\right.$ is not forbidden by general considerations. However, such terms always drop out in the calculation of the transport current, which interest us here (see Sec. IIE). This happens because in all required expressions, the renormalized Liouvillian $\bar{L}$, parametrized as (136), is evaluated between two $\bar{G}$ vertices [cf. Eq. (79)]. Therefore, by Eq. (125) the term $\left.\mid Z_{R}\right)\left(Z_{L} \mid\right.$ with coefficient $\zeta$ always drops out. We emphasize, however, that $\zeta$ does enter into the stationary state [cf. Eq. (151)] and other physical quantities, such as the average dot energy, and may therefore be important, e.g., for thermal transport problems.

(iv) Terms of the form $\left.\mid \alpha_{\eta, \sigma}^{+}\right)(\bullet \mid$ and $\mid \bullet)\left(\alpha_{\eta, \sigma}^{-} \mid\right.$can appear only in the bare Liouvillian $L=[H, \bullet]_{-}$or the infinitetemperature kernel $\tilde{\Sigma}=-i \frac{1}{2} \Gamma_{1} \bar{G}_{1} \tilde{G}_{\overline{1}}$, but not in the nontrivial kernel $\bar{\Sigma}(z)$ :

$$
\begin{aligned}
& \left(\alpha_{\eta, \sigma}^{+} \mid \bar{\Sigma}(z)=0,\right. \\
& \left.\bar{\Sigma}(z) \mid \alpha_{\eta, \sigma}^{-}\right)=0 .
\end{aligned}
$$

Both relations follow from the fact that $\bar{\Sigma}(z)$ contains only vertices of the type $\bar{G}$ in the expansion (79). Equation (132) follows from Eq. (121) applied to Eq. (79) and then using $\left(Z_{L} \mid \bar{L}=0\right.$ and $\left(Z_{L} \mid \bar{G}=0\right.$, the vacuum property (85). Analogously, Eq. (133) follows from Eq. (122) using our general result (130), $\left.\bar{L} \mid Z_{R}\right)=-4 i \Gamma \mid Z_{R}$ ), and $\left.\bar{G} \mid Z_{R}\right)=0$, Eq. (86). Equations (132) and (133) allow us to make general predictions about the excitation spectrum of the exact dot Liouvillian $L(z)$ see Secs. II D3 and III B4.

b. Spin- and charge-rotation symmetry. We now first expand the infinite-temperature self-energy $\tilde{\Sigma}$ and the renormalized Liouvillian $\bar{L}$ in the basis (91)-(96). Substituting the above bra-ket expansions of the superoperators $\bar{G}_{1}$ and $\tilde{G}_{\overline{1}}$ into Eq. (71) we get

$$
\begin{aligned}
\tilde{\Sigma}= & -i \Gamma\left[4 \mid Z_{R}\right)\left(Z_{R}\left|+2 \sum_{\sigma= \pm}\right| \chi_{\sigma}\right)\left(\chi_{\sigma} \mid\right. \\
& \left.+2 \sum_{\sigma= \pm} \mid T_{\sigma}\right)\left(T_{\sigma}\left|+2 \sum_{\sigma= \pm}\right| S_{\sigma}\right)\left(S_{\sigma} \mid\right. \\
& \left.+\sum_{\sigma= \pm} \sum_{\eta= \pm} \sum_{\nu= \pm}(2-v) \mid \alpha_{\eta, \sigma}^{v}\right)\left(\alpha_{\eta, \sigma}^{v} \mid\right]
\end{aligned}
$$

Clearly, $\tilde{\Sigma}$ is explicitly anti-Hermitian in the superoperator sense [cf. Eq. (72)]. Combining this with the bare dot Liouvillian, obtained by expanding the commutator $L=[H, \bullet]_{-}$ of (102), we obtain

$$
\begin{aligned}
\left.\bar{L}_{\Lambda}\right|_{\Lambda=\infty}:= & \left.\bar{L}=L+\tilde{\Sigma}=-i 4 \Gamma \mid Z_{R}\right)\left(Z_{R} \mid-2 i \Gamma \chi_{0}\right. \\
& +\sum_{\sigma}\left\{(\sigma B-2 i \Gamma) \mid S_{\sigma}\right)\left(S_{\sigma} \mid+[\sigma(U+2 \epsilon)\right. \\
& \left.-2 i \Gamma] \mid T_{\sigma}\right)\left(T_{\sigma} \mid+\sum_{\eta, v}\left[\eta\left(\epsilon+\frac{U}{2}\right)+\sigma \frac{B}{2}\right.\right. \\
& \left.-i(2-v) \Gamma] \mid \alpha_{\eta, \sigma}^{\nu}\right)\left(\alpha_{\eta, \sigma}^{\nu}\left|+\frac{U}{2} \sum_{\eta, v}\right| \alpha_{\eta, \sigma}^{\nu}\right)\left(\alpha_{\eta, \sigma}^{\bar{\nu}} \mid\right\},
\end{aligned}
$$

where for later reference we introduced the notation $\left.\bar{L}_{\Lambda}\right|_{\Lambda=\infty}:=\bar{L}$ of Sec. III. All nonzero eigenvalues of $\bar{L}$ have negative imaginary parts, thereby automatically regularizing all resolvents that can appear $\bar{G}(z-\bar{L}-X)^{-1} \bar{G}$ in the perturbation theory for $\bar{\Sigma}(z)$ for $z \rightarrow i 0$. This can be seen explicitly since all terms are already in diagonal superoperator form, with the exception of the odd-fermion terms [the last two lines in Eq. (135)], the eigenvalues of which are given below [set $\Delta F_{\eta, \sigma}^{-,+}=0$ in Eq. (163)]. Note that the right zero eigenvector of $\bar{L}_{\Lambda=\infty}$ is $\frac{1}{2} \mid Z_{L}$ ) [ it is the only basis vector missing in Eq. (135)], in agreement with the result (73) in Sec. II B4. We further note that the infinite-temperature kernel $\tilde{\Sigma}$ (134) contributes terms to Eq. (135) that are diagonal in the index $v$, whereas contributions off diagonal in $v$ are produced in Eq. (135) by the Coulomb interaction included in the bare dot Liouvillian $L$. We will show in Sec. III B4 that the continuous RT-RG produces only contributions to the effective Liouvillian $L(z)$ that are off diagonal in $v$, which has important consequences.

We can now write the exact form of the QD effective Liouvillian $L(z)$ taking into account all general restrictions that we have derived above. In the most general case that we consider, only spin- and charge-rotation symmetry about the $z$ axis: this implies that the effective Liouvillian must be a sum of superoperators that (i) transform as an irreducible tensor of any rank but with index zero with respect to both the charge- and spin-rotation group (i.e., by pairing only bras and kets of basis supervectors with the same charge and spin indices) and (ii) preserve the fermion parity (i.e., by avoiding combinations of fermion and boson kets and bras). Using Table I, which lists the transformation properties of the basis supervectors (91)-(96), we can readily construct the most general form of superoperators of this kind, which are furthermore compatible with the causal structure of the perturbative series (68). In Table II, we have classified all these superoperators according to their irreducible transformation properties under the full symmetry group of both spin and charge rotations. This makes it easy to impose further restrictions on the expansion coefficients in the special cases of higher symmetry ( $B=0$ and/or $\epsilon=-U / 2, \mu_{L}=\mu_{R}$ ) (see Sec. II D4). The most general form of the exact QD effective 
TABLE II. Irreducible tensor superoperators (ITSOs) of different rank but with (i) zero index with respect to both spin and charge rotations and (ii) satisfying the causal structure constraints (cf. Sec. II C5a). The general effective Anderson Liouvillian (136) is a linear combination of all of these, where the coefficient of $\left.\mid Z_{R}\right)\left(Z_{R} \mid\right.$ is always fixed to $-4 i \Gamma$ in the wide-band limit (see Sec. II C5a). In the special limits of higher symmetry only $(0,0), S$-ITSO resp. $T$-ITSOs can appear in this expansion (see Sec. II D4). The ITSOs are constructed by standard angular momentum coupling. For this, one takes the supervectors in Table I, denoted schematically by $\left.\mid s, m_{s} ; t, m_{t}\right)$ where $s, t$ and $m_{s}, m_{t}$ are the rank and index with respect to spin and charge rotations in Liouville space. Then, one constructs conjugate bra supervectors that transform with the same rank and index: these are $(-1)^{s-m_{s}+t-m_{t}}\left(s,-m_{s} ; t,-m_{t} \mid\right.$. Coupling these kets and bras with Clebsch-Gordan coefficients gives the superoperators transforming with definite rank and index with respect to spin and charge rotations.

\begin{tabular}{lcc}
\hline \hline Superoperator & $\begin{array}{c}S \text {-ITSO } \\
\text { (rank,index) }\end{array}$ & $\begin{array}{c}T \text {-ITSO } \\
\text { (rank, index) }\end{array}$ \\
\hline $\left.\mid Z_{R}\right)\left(Z_{R}|,| Z_{R}\right)\left(Z_{L} \mid\right.$ & $(0,0)$ & $(0,0)$ \\
$\left.\sum_{m=0, \pm 1} \mid S_{m}\right)\left(S_{m} \mid\right.$ & $(0,0)$ & $(0,0)$ \\
$\left.\sum_{m=0, \pm 1} \mid T_{m}\right)\left(T_{m} \mid\right.$ & $(0,0)$ & $(0,0)$ \\
$\left.\mid S_{0}\right)\left(Z_{L}|,| Z_{R}\right)\left(S_{0} \mid\right.$ & $(1,0)$ & $(0,0)$ \\
$\left.\sum_{m= \pm 1} m \mid S_{m}\right)\left(S_{m} \mid\right.$ & $(1,0)$ & $(0,0)$ \\
$\left.\mid T_{0}\right)\left(Z_{L}|,| Z_{R}\right)\left(T_{0} \mid\right.$ & $(0,0)$ & $(1,0)$ \\
$\left.\sum_{m= \pm 1} m \mid T_{m}\right)\left(T_{m} \mid\right.$ & $(0,0)$ & $(1,0)$ \\
$\left.\mid S_{0}\right)\left(T_{0}|,| T_{0}\right)\left(S_{0} \mid\right.$ & $(1,0)$ & $(1,0)$ \\
$\left.\sum_{\sigma, \eta= \pm} \sigma^{\tau_{\sigma}} \eta^{\tau_{\eta}} \mid \alpha_{\eta, \sigma}^{v}\right)\left(\alpha_{\eta, \sigma}^{v^{\prime}} \mid\right.$ & $\left(\tau_{\sigma}, 0\right)$ & $\left(\tau_{\eta}, 0\right)$ \\
$\left.\sum_{m=0, \pm 1}\left(3 m^{2}-2\right) \mid S_{m}\right)\left(S_{m} \mid\right.$ & $\tau_{\sigma}=0,1$ & $\tau_{\eta}=0,1$ \\
$\left.\sum_{m=0, \pm 1}\left(3 m^{2}-2\right) \mid T_{m}\right)\left(T_{m} \mid\right.$ & $(2,0)$ & $(0,0)$ \\
\hline \hline
\end{tabular}

Liouvillian $L(z)=L+\Sigma(z)=\bar{L}+\bar{\Sigma}(z)$ then reads as

$$
\begin{aligned}
i L(z)= & \left.4 \Gamma \mid Z_{R}\right)\left(Z_{R}|+\zeta| Z_{R}\right)\left(Z_{L}|+| Z_{R}\right)(\vec{\phi}|+| \vec{\psi})\left(Z_{L} \mid\right. \\
& +\xi+\sum_{\sigma}\left[M_{\sigma} \mid T_{\sigma}\right)\left(T_{\sigma}\left|+E_{\sigma}\right| S_{\sigma}\right)\left(S_{\sigma} \mid+\sum_{\eta} F_{\eta, \sigma}\right],
\end{aligned}
$$

where $\Gamma=\frac{1}{2} \sum_{r} \Gamma_{r}$ and $F_{\eta, \sigma}$ are superoperators acting in the two-dimensional $v$ space spanned by $\mid \alpha_{\eta, \sigma}^{v}$ ) (see below). This is a central result of the paper, and before discussing the occurring coefficients in detail, we point out its importance.

By exploiting only its general properties we have reduced the number of terms contributing to $L(z)$ from 256 [cf. Eq. (82)] down to just 30 . The key simplification came by using the causal field superoperators to construct the Liouville Fock space. The resulting general Liouvillian can be easily diagonalized as we show in the next section. Furthermore, because of its general nature, the parametrization (136) is useful in applications other than those considered here and may be extended to more complex Anderson-type models (see Appendix E). For example, we note that $\left.\mid Z_{R}\right)$ is always a right eigensupervector of the effective Liouvillian decaying with rate $2 m \Gamma$ where $m$ is the number of electrodes attached to the dot (for our case $m=2$ ). This mode was recently also found in a study investigating the time relaxation of the density matrix of the Anderson model. ${ }^{39}$ It was observed that this mode, appearing in one-loop perturbation theory, is not affected by two-loop corrections. Our work generalizes this result: the eigenvalue of the right eigensupervector $\left.\mid Z_{R}\right)$ is not affected by any higher-order corrections. We also see how this relies on assuming the wide-band limit. Both insights directly rely on the causal representation of the field superoperators. Further implications for the time dependence will be discussed elsewhere. ${ }^{38,50}$

We now list how the expansion coefficients for $L(z)$ are incorporated in Eq. (136) through the following terms:

(i) The zero eigensupervectors of the vertices $\left.\mid Z_{L}\right)$ and $\left.\mid Z_{R}\right)$ : the choice of their coefficients is based on the general properties of the perturbative series (cf. Sec. II B3).

(ii) A supervector in the two-dimensional $\chi$ subspace spanned by $\left.\mid \chi_{\sigma}\right)$ :

$$
\left.\mid \vec{\psi})=\sum_{\sigma} \psi_{\sigma} \mid \chi_{\sigma}\right)
$$

(iii) An independent vector in the corresponding dual space

$$
(\vec{\phi})=\sum_{\sigma} \phi_{\sigma}\left(\chi_{\sigma}\right)
$$

(iv) A superoperator acting on the $\chi$ subspace

$$
\left.\xi=\sum_{\sigma, \sigma} \xi_{\sigma, \sigma^{\prime}} \mid \chi_{\sigma}\right)\left(\chi_{\sigma^{\prime}} \mid=\sum_{i=0,1,2,3} \xi_{i} \chi_{i} .\right.
$$

Here, after the second equality, the matrix $\xi_{\sigma, \sigma^{\prime}}$ is decomposed in the standard basis $\left(\tau_{i}\right)_{\sigma, \sigma^{\prime}}$ of the unit $(i=0)$ and three Pauli matrices $(i=1,2,3)$, giving another $\chi$-subspace superoperator basis:

$$
\left.\chi_{i}=\sum_{\sigma, \sigma^{\prime}}\left(\tau_{i}\right)_{\sigma, \sigma^{\prime}} \mid \chi_{\sigma}\right)\left(\chi_{\sigma^{\prime}} \mid\right.
$$

(v) Four superoperators acting on the two-dimensional $\alpha_{\eta, \sigma}$ subspaces spanned by $\left.\mid \alpha_{\eta, \sigma}^{ \pm}\right)$:

$$
\begin{aligned}
F_{\eta, \sigma} & \left.=\sum_{\nu, v^{\prime}} F_{\eta, \sigma}^{v, v^{\prime}} \mid \alpha_{\eta, \sigma}^{v}\right)\left(\alpha_{\eta, \sigma}^{v^{\prime}} \mid\right. \\
& =\sum_{i=0,1,2,3} F_{\eta, \sigma}^{i} \alpha_{\eta, \sigma}^{i}
\end{aligned}
$$

with unit and Pauli-vector superoperators

$$
\left.\alpha_{\eta, \sigma}^{i}=\sum_{\nu, v^{\prime}}\left(\tau_{i}\right)_{v, v^{\prime}} \mid \alpha_{\eta, \sigma}^{\nu}\right)\left(\alpha_{\eta, \sigma}^{v^{\prime}} \mid\right.
$$

for each fixed $\sigma= \pm$ and $\eta= \pm$.

It is convenient to use the four-vector as well as the $2 \times 2$ matrix representations for the superoperators $\xi$ and $F_{\eta, \sigma}$.

All the above expansion coefficients depend on the QD frequency $z$ (not written) and satisfy the following conjugation relations, which derive from the Hermiticity condition (70): $K[i L(z)] K=i L\left(-z^{*}\right)$. For the charge- and spin-diagonal operators, these are

$$
\vec{\phi}(z)=\vec{\phi}^{*}\left(-z^{*}\right), \quad \vec{\psi}(z)=\vec{\psi}^{*}\left(-z^{*}\right), \quad \xi(z)=\xi^{*}\left(-z^{*}\right),
$$

implying that these coefficients are real only for zero frequency $z=i 0$. For the charge- or spin-nondiagonal operators 
we have

$$
\begin{gathered}
F_{\eta, \sigma}^{v, v^{\prime}}(z)=v v^{\prime} F_{\bar{\eta}, \bar{\sigma}}^{v, \nu^{\prime}}\left(-z^{*}\right), \quad v, v^{\prime}= \pm \\
M_{\sigma}(z)=M_{\bar{\sigma}}^{*}\left(-z^{*}\right), \\
E_{\sigma}(z)=E_{\bar{\sigma}}^{*}\left(-z^{*}\right) .
\end{gathered}
$$

Therefore, at finite dot frequency $z$, all parameters are in general complex and all $2 \times 2$ coefficient matrices are nonHermitian.

Note that in Eq. (136) we have parametrized $i L(z)$, rather than $L(z)$, i.e., including the imaginary factor $i$. This anticipates the application to the RT-RG, where a renormalized version of the Liouvillian $\bar{L}$ appears in the final RG equations only as the combination $i \bar{L}$ (cf. Secs. IIIC1 and IIID1). Finally, we emphasize that the simplifications that led up to the parametrization Eq. (136) remain valid for the RT-RG: Eq. (69), as well as Eqs. (85) and (86) do not change under the continuous renormalization, as will be shown in Sec. III.

\section{Effective Liouvillian}

\section{Spectral decomposition of $L(z)$ and $\bar{L}$}

Above we have reduced $L(z)$ to block-diagonal form as far as possible by using symmetry and general properties. In Sec. III, we will see that $L(z)$ is closely related to a renormalized version of the QD Liouvillian $\bar{L}$ that we will denote by $\bar{L}_{\Lambda}$. To make this clear, we have to anticipate a result: $\bar{L}_{\Lambda}$ interpolates between $\bar{L}$ and $L(z)$ as the flow parameter $\Lambda$ varies from $\infty$ to $0: L(z)=\bar{L}+\int_{\infty}^{0} d \Lambda \frac{d \bar{L}_{\Lambda}}{d \Lambda}$. This is done by redistributing diagrams of $\bar{\Sigma}$ in Eq. (80) and including a fraction of them into a redefinition of the Liouvillian $\bar{L}$. By construction, $\bar{\Sigma}(z)$ is thus decomposed into "pieces" $\frac{d \bar{L}_{\Lambda}}{d \Lambda}$ with the same matrix structure that are accumulated during the flow. At the end of the flow, $\bar{L}_{\Lambda}$ equals $L(z)$. Therefore, $\bar{L}_{\Lambda}$ has an expansion of the same form [Eq. (136)] as $L(z)$. For notational simplicity, we denote the expansion coefficients of $\bar{L}_{\Lambda}$ by the same variables as for $L(z)$. In cases where this leads to confusion, the coefficients of $\bar{L}_{\Lambda}$ are distinguished from those of $L(z)$ by indicating their $\Lambda$ dependence, e.g., $F_{\eta \sigma \Lambda}^{v, v^{\prime}}$ versus $F_{\eta \sigma}^{v, v^{\prime}}$, which can, however, often be omitted. All results obtained in this section thus apply to both the exact $L(z)$ as well as the renormalized $\bar{L}_{\Lambda}$. This explicit form of $\bar{L}_{\Lambda}$ is required since later on it needs to be inserted into resolvent superoperators that appear in the RG equations.

We therefore need to completely diagonalize $\bar{L}_{\Lambda}$ such that it can be expanded into its eigenprojectors $\left.P^{k}=\mid \bar{\lambda}^{k}\right)\left(\lambda^{k} \mid=\right.$ $\left(P^{k}\right)^{2}$ :

$$
\bar{L}_{\Lambda}=\sum_{k} \lambda^{k} P^{k},
$$

where the sum runs over the labels $k$ of the eigenvalues. Here, $\left(\lambda^{k} \mid\right.$ and $\left.\mid \bar{\lambda}^{k}\right)$ are the left and right eigensupervectors of $\bar{L}_{\Lambda}$ for the same eigenvalue $\lambda^{k}: \bar{L}_{\Lambda} P^{k}=P^{k} \bar{L}_{\Lambda}=\lambda^{k} P^{k}$. Using this complete and orthogonal set of projectors, one can then evaluate resolvent superoperators in Eq. (79) explicitly:

$$
\cdots \bar{G} \frac{1}{z-X-\bar{L}_{\Lambda}} \bar{G} \cdots=\cdots \sum_{i} \frac{1}{z-X-\lambda^{i}} \bar{G} P^{i} \bar{G} \cdots .
$$

We note that the diagonalization of Eq. (136) can also be useful for higher orders of (renormalized) perturbation theory Eq. (68) [Eq. (79)]: when expanding the QD $L$ and $\bar{L}_{\Lambda}$ in the form (136) it is directly adapted to all symmetries of the problem and one can efficiently construct explicit matrix representation of the self-energies $\Sigma(z)$ and $\bar{\Sigma}(z)$, respectively.

The application of the above spectral decomposition to the continuous RG in Sec. III involves two assumptions that should be pointed out here. First, we always assume that the zero eigenvalue of $\bar{L}_{\Lambda}$ is nondegenerate, corresponding to the unique stationary state for the density operator. This is always found to be the case for the numerically calculated RG flows discussed in Sec. III. However, in principle, it may also happen that two (or more) nonzero eigenvalues of $\bar{L}_{\Lambda}$ become degenerate during this flow. If this is the case, and additionally the supermatrix $\bar{L}_{\Lambda}$ has nonzero elements on its diagonal in the normal Jordan form in the degenerate subspace, then no complete eigenprojector basis exists. For the Anderson model, this presents no crucial complication: the eigenbasis can in principle be circumvented for the calculation of the two-dimensional superoperators. However, numerically we never meet this problem in the application of the RT-RG theory presented below.

\section{Eigenvalues, eigensupervectors, and the stationary state}

We now explicitly diagonalize $L(z)$ or $\bar{L}_{\Lambda}$, parametrized as in Eq. (136), in each of its block diagonals. We first list the eigenvalues and projectors in the block spanned by the chargeand spin-diagonal bosonic operators $\left.\left(Z_{L}\right), \mid Z_{R}\right),\left(S_{0}\right)$, and $\left.\mid T_{0}\right)$, which contain the stationary nonequilibrium state:

(a) Eigenvalue $\lambda^{Z_{L}}=0$ with projector

$$
\left.P^{Z_{L}}=2 \mid \rho\right)\left(Z_{L} \mid,\right.
$$

with the stationary density operator

$$
\left.\left.\left.\mid \rho)=-\frac{1}{2 \xi} \mid \vec{\psi}\right)+\frac{1}{2} \mid Z_{L}\right)-\frac{\zeta-\left(\vec{\phi}\left|\xi^{-1}\right| \vec{\psi}\right)}{8 \Gamma} \mid Z_{R}\right) .
$$

We note that the coefficient $\zeta$ appears only in the stationary state and the next projector $P^{Z_{R}}$, but not in any other eigenprojector or eigenvalue.

(b) Eigenvalue $\lambda^{Z_{R}}=-i 4 \Gamma$ with projector

$$
\begin{aligned}
P^{Z_{R}}= & \left.\left(\vec{\phi}\left|\frac{1}{4 \Gamma(4 \Gamma-\xi)}\right| \vec{\psi}\right) \mid Z_{R}\right)\left(Z_{L} \mid\right. \\
& \left.+\mid Z_{R}\right)\left(Z_{R}\left|+\frac{\zeta}{4 \Gamma}\right| Z_{R}\right)\left(Z_{L}|+| Z_{R}\right)\left(\vec{\phi} \mid \frac{1}{4 \Gamma-\xi} .\right.
\end{aligned}
$$

(c) Eigenvalues $\lambda^{{ }^{ \pm}}$with projectors

$$
\begin{aligned}
P^{3,4}= & \left.P^{\chi, \pm}+\frac{\left(\vec{\phi}\left|P^{\chi \pm}\right| \vec{\psi}\right)}{\left(\lambda^{\chi \pm}-4 \Gamma\right) \lambda \chi^{ \pm}} \mid Z_{R}\right)\left(Z_{L} \mid\right. \\
& \left.+\frac{1}{\lambda^{\chi, \pm}-4 \Gamma} \mid Z_{R}\right)\left(\vec{\phi}\left|P^{\chi, \pm}+\frac{1}{\lambda^{\chi, \pm}} P^{\chi \pm}\right| \vec{\psi}\right)\left(Z_{L} \mid .\right.
\end{aligned}
$$

The eigenvalues $\lambda^{\chi \pm}$ are determined by first diagonalizing $\xi$ in the $\chi$ subspace, i.e., by finding eigenprojectors $P^{\chi, \sigma}$ of $\xi$ :

$$
\xi P^{\chi, \sigma}=P^{\chi, \sigma} \xi=\lambda^{\chi, \sigma} P^{\chi, \sigma} .
$$


Since $\xi_{\sigma, \sigma^{\prime}}$ is a $2 \times 2$ non-Hermitian matrix, it can be expressed in the vector $\boldsymbol{\xi}=\left(\xi_{1}, \xi_{2}, \xi_{3}\right)$ and coefficient $\xi_{0}$ [cf. Eq. (139)], all of which are complex:

$$
\begin{aligned}
& \lambda^{\chi, \sigma}=-i\left(\xi_{0}+\sigma \sqrt{\xi^{2}}\right), \\
& P^{\chi, \sigma}=\frac{1}{2} \chi_{0}+\sigma \frac{\chi \cdot \xi}{2 \sqrt{\xi^{2}}} .
\end{aligned}
$$

Here, the square root of the complex argument is defined such that the branch cut lies in the lower half complex plane since the effective Liouvillian must be regular in the upper half-plane according to Eqs. (32) and (35).

The remaining block diagonals acting on bosonic subspaces are one dimensional: for $\sigma= \pm$,

$$
\begin{array}{ll}
\lambda^{T_{\sigma}}=-i M_{\sigma}, & \left.P^{T_{\sigma}}=\mid T_{\sigma}\right)\left(T_{\sigma} \mid,\right. \\
\lambda^{S_{\sigma}}=-i E_{\sigma}, & \left.P^{S_{\sigma}}=\mid S_{\sigma}\right)\left(S_{\sigma} \mid .\right.
\end{array}
$$

Finally, in the four remaining, two-dimensional fermionic subspaces labeled by $\alpha_{\eta, \sigma}$ (for fixed $\eta$ and $\sigma$ ), the eigenvalues and projectors can be calculated in the same way as for the bosonic $\chi$ block:

$$
\begin{aligned}
\lambda^{\alpha_{\eta, \sigma}, \pm} & =-i\left(F_{\eta, \sigma}^{0} \pm \sqrt{\mathbf{F}_{\eta \sigma}^{2}}\right) \\
P^{\alpha_{\eta, \sigma}, \pm} & =\frac{1}{2} \alpha_{\eta, \sigma}^{0} \pm \frac{\mathbf{F}_{\eta \sigma} \cdot \boldsymbol{\alpha}_{\eta, \sigma}}{2 \sqrt{\mathbf{F}_{\eta \sigma}^{2}}}
\end{aligned}
$$

where the coefficients $F_{\eta \sigma}^{0}$ and $\mathbf{F}_{\eta \sigma}=\left(F_{\eta \sigma}^{1}, F_{\eta \sigma}^{2}, F_{\eta \sigma}^{3}\right)$ are again complex [cf. Eq. (142)].

We note that it is in principle possible that $\xi^{2}=0$ while $\xi \neq \overrightarrow{0}$. In this case, the supermatrix representation of $\chi$ has nonzero diagonal element in its normal Jordan form. In this case, $\xi$ has no eigenbasis and Eq. (156) does not apply. Still, the required matrix valued functions of $\xi$ can be calculated using the Hamilton-Cayley theorem. The same remarks apply to $\mathbf{F}_{\eta \sigma}$ and Eq. (160). However, in practical applications, we never meet such a situation. We also note that during the RG flows discussed in Sec. III, we never run into points where $\lambda^{\chi, \pm}=-i 4 \Gamma$ and one therefore need not worry about the vanishing of the denominators in Eq. (153) or the existence of the inverse of $(4 \Gamma-\xi)$ in Eq. (152).

An important simplification applies to the first four bosonic projectors $P^{Z_{L}}, P^{Z_{R}}, P^{3,4}$ that involve the vectors $\mid Z_{L}$ ) and $\left(Z_{R}\right)$, analogous to the corresponding terms in the expansion (136) of the effective Liouvillian (cf. Sec. II C5a). When inserting projectors into Eq. (149) between two vertices $\bar{G}$, (i) the projectors $P^{Z_{L}}, P^{Z_{R}}$ give no contributions; (ii) the projectors $P^{3,4}$ only contribute through the first term $P^{\chi, \pm}$ in Eq. (153).

As a result, in all applications below we can replace Eq. (148) with the simpler expansion

$\bar{L}_{\Lambda} \stackrel{\bar{G} \ldots \bar{G}}{\longrightarrow} \lambda^{\chi} P^{\chi}+\sum_{\sigma}\left[\lambda^{S_{\sigma}} P^{S_{\sigma}}+\lambda^{T_{\sigma}} P^{T_{\sigma}}+\sum_{\eta} \lambda^{\alpha_{\eta, \sigma}} P^{\alpha_{\eta, \sigma}}\right]$.

Here, we leave implicit the sum over the two eigenvalues $\lambda^{\chi, \pm}$ and $\lambda^{\alpha_{\eta, \sigma}, \pm}$ in the $\chi$ and $\alpha_{\eta, \sigma}$ subspaces, respectively. A crucial stability requirement for the RG in Sec. III is thereby explicitly satisfied: the zero eigenprojector (150), corresponding to the physical stationary state, never appears in the resolvents.

\section{Fermionic excitations: Spectral decomposition of $\bar{\Sigma}$}

The expansion (136) can of course also be applied to $\bar{\Sigma}(z)=$ $L(z)-\bar{L}$. This, however, involves additional simplifications causing certain terms appearing in the expansion of $L(z)$ (and $\left.\bar{L}_{\Lambda}\right)$ to be absent. First, due to Eq. (131), the term $\left.\mid Z_{R}\right)\left(Z_{R} \mid\right.$ is missing. Second, since Eqs. (132) and (133) derive from the causal structure (cf. Sec. II C5a), most of the coefficients of the fermionic sector of $\bar{\Sigma}$, denoted by $-i \Delta F_{\eta \sigma}^{v, v^{\prime}}$, vanish: $\Delta F_{\eta \sigma}^{v, v^{\prime}}=$ $\delta_{\nu,-} \delta_{v^{\prime},+} \Delta F_{\eta \sigma^{\prime}}^{-,+}$. We can express the coefficient matrices $F_{\eta \sigma}^{\nu, v^{\prime}}$ of $L(z)=\bar{L}+\bar{\Sigma}(z)$ using Eq. (135), in terms of those of $\bar{\Sigma}(z)$, i.e., in terms of the $\Delta F_{\eta \sigma}^{-,+}$:

$$
\begin{aligned}
-i & \left(\begin{array}{cc}
F_{\eta \sigma}^{+,+} & F_{\eta \sigma}^{+,-} \\
F_{\eta \sigma}^{-,+} & F_{\eta \sigma}^{-,-}
\end{array}\right) \\
& =\left(\begin{array}{cc}
\eta\left(\epsilon+\frac{U}{2}\right)+\sigma \frac{B}{2}-i \Gamma & \frac{U}{2} \\
\frac{U}{2}-i \Delta F_{\eta \sigma}^{-,+} & \eta\left(\epsilon+\frac{U}{2}\right)+\sigma \frac{B}{2}-i 3 \Gamma
\end{array}\right) .
\end{aligned}
$$

Converting to spherical coefficients $F_{\eta, \sigma}^{0}$ and $\mathbf{F}_{\eta \sigma}$ and using Eq. (159), we find

$$
\begin{gathered}
\lambda^{\alpha_{\eta, \sigma}, \pm}=\eta\left(\epsilon+\frac{U}{2}\right)+\sigma \frac{B}{2}-2 i \Gamma \\
\pm \eta \sqrt{\frac{U^{2}}{4}-\Gamma^{2}-i \frac{U \Delta F_{\eta, \sigma}^{-,+}}{2}}, \\
P^{\alpha_{\eta, \sigma}, \pm}=\frac{\alpha_{\eta, \sigma}^{0}}{2} \pm \eta \frac{\frac{U}{2} \alpha_{\eta, \sigma}^{1}+i \Gamma \alpha_{\eta, \sigma}^{3}-i \Delta F_{\eta, \sigma}^{-,+} \alpha_{\eta, \sigma}^{-}}{2 \sqrt{\frac{U^{2}}{4}-\Gamma^{2}-i \frac{U \Delta F_{\eta, \sigma}^{-,+}}{2}}} .
\end{gathered}
$$

We thus find that the functional form of the fermionic eigenvalues of $L(z)$ is severely restricted by the casual structure of $\bar{\Sigma}$ : all the frequency-dependent renormalization effects enter into the projectors and eigenvalues solely through the four complex numbers $\Delta F_{\eta, \sigma}^{-,+}$in the four $\alpha_{\eta \sigma}$ blocks $(\eta, \sigma= \pm)$.

For a QD decoupled from the reservoirs, $\Gamma=0$, we have $\tilde{\Sigma}=0=\bar{\Sigma}(z)=0$ and thus $\Delta F_{\eta, \sigma}^{-,+}=0$ :

$$
\begin{gathered}
\lambda^{\alpha_{\eta, \sigma},+}=\eta(\epsilon+U)+\sigma B / 2, \\
\lambda^{\alpha_{\eta, \sigma},-}=\eta \epsilon+\sigma B / 2 .
\end{gathered}
$$

This is the spectrum of many-body energy excitations when adding a single electron, starting from either an empty QD $\left(\lambda^{\alpha_{\eta, \sigma},+}\right)$ or a singly occupied QD with spin $\bar{\sigma}\left(\lambda^{\alpha_{\eta, \sigma},-}\right)$. This is confirmed by the eigenprojectors in the limit $\Gamma \rightarrow 0$ for finite $U$ :

$$
P^{\alpha_{\eta, \sigma}, \pm}=\frac{1}{2}\left(\alpha_{\eta, \sigma}^{0} \pm \alpha_{\eta, \sigma}^{1}\right)= \begin{cases}\mid \sigma, 0)(\sigma, 0 \mid & +, \\ \mid 2, \bar{\sigma})(2, \bar{\sigma} \mid & -,\end{cases}
$$

where $\mid \sigma, 0)=|\sigma\rangle\langle 0|=\left(1 / 2-Z_{R}\right) d_{\sigma}^{\dagger}$ and $\left.\mid 2, \bar{\sigma}\right)=|2\rangle\langle\bar{\sigma}|=$ $\sigma\left(1 / 2+Z_{R}\right) d_{\sigma}^{\dagger}$. These are the virtual intermediate states and energies that enter through $L$ into the perturbation expansion (68) for $\Sigma(z)$.

For any finite coupling $\Gamma$, but infinite temperature, we have $\bar{\Sigma}=0$ and therefore again $\Delta F_{\eta, \sigma}^{-,+}=0$. In this case, however, 




FIG. 3. (Color online) Fermionic excitation energies and widths of the infinite-temperature Liouvillian $\bar{L}$ plotted as function of the tunnel coupling strength $\Gamma / U$ for $\epsilon=B=0$. The energy and width are given by the real and imaginary parts of the fermionic eigenvalues $\lambda^{\alpha_{\eta, \sigma}, v}$ [Eq. (163) with $\Delta F_{\eta, \sigma}^{-,+}=0$ ]. The real parts for $v=+$ (red) and $v=-1$ (green), respectively, are given by the full lines, and the imaginary parts are indicated by the shaded width of the level with the corresponding color. For $U>2 \Gamma$, the excitations have different energies, split by $U$, but with the same width $2 \Gamma$, whereas for $2 \Gamma>U$ they have the same energies but different widths: for $\Gamma \gg U-\operatorname{Im} \lambda^{\alpha_{\eta, \sigma}, \pm} \approx \Gamma$ (red) $3 \Gamma$ (green).

$\tilde{\Sigma} \propto \Gamma \neq 0$ and the eigenvalues obtained from Eq. (163) depend qualitatively on the interaction strength $U$ : for $U<$ $2 \Gamma$, the coupling to the reservoirs $\Gamma$ adds different imaginary parts to the degenerate real eigenvalues $\eta(\epsilon+U / 2)+\sigma B / 2$, whereas for $U>2 \Gamma$ it adds a uniform imaginary part $-i \Gamma$ to these eigenvalues while differently shifting their real parts. This dependence on $U$ is plotted in Fig. 3 and shows a bifurcation at $U=2 \Gamma$. These are the energies and projectors that enter through $\bar{L}$ into the renormalized perturbation theory (79).

Finally, for both finite coupling $\Gamma$ and finite temperature $T$, the complex coefficients $\Delta F_{\eta, \sigma}^{-,+}$are nontrivial functions that need to be calculated, e.g., either perturbatively or using the RG (see Sec. III B4). However, even in this case, Eq. (163) provides an exact relation: the average of the complex particle and hole excitation eigenvalues in each $\alpha_{\eta \sigma}$ block is independent of $\Delta F_{\eta, \sigma}^{-,+}$and thereby, also independent of the frequency $z$ :

$$
\frac{1}{2} \sum_{ \pm} \lambda^{\alpha_{\eta, \sigma} \pm}=\eta\left(\epsilon+\frac{U}{2}\right)+\sigma \frac{B}{2}-2 i \Gamma .
$$

Physically speaking, both the renormalized energies of singleparticle fermionic excitation energies (real parts) as well as their decay rates/broadenings (imaginary parts) lie symmetric with respect to the above average values. For example, if the particle excitation broadens, the hole excitation must sharpen up and vice versa.

Finally, we can infer an important physical stability constraint on the functions $\Delta F_{\eta, \sigma}^{-,+}$: they must be such that the imaginary part of the square root is less than $2 \Gamma$ for all $\Lambda$ and $z$. Otherwise, inverse Laplace transforming $L(z)$ to time space would yield terms that diverge for $t \rightarrow \infty$, which is unphysical. This restricts the maximal excitation widths in the fermionic block: the negative imaginary part of the eigenvalues $\lambda^{\alpha_{\eta, \sigma}, \pm}$ can not exceed the value $4 \Gamma$.

\section{High-symmetry stationary states}

As a cross-check on the results of Sec. IID2, we now analyze the stationary density operator in the two special parameter regimes where the model has a higher symmetry than in general. In both cases, the superoperator $\xi$ has no off-diagonal terms in the basis of $\left.\mid S_{0}\right)$ and $\left.\mid T_{0}\right)$,

$$
\left.\xi=\xi_{T T} \mid T_{0}\right)\left(T_{0}\left|+\xi_{S S}\right| S_{0}\right)\left(S_{0} \mid,\right.
$$

because there are no scalars with respect to spin or charge rotations that contain such terms as components (see Table II). If either symmetry is broken, charge and/or spin rotations allow for mixing terms $\left.\mid T_{0}\right)\left(S_{0} \mid\right.$ and $\left.\mid S_{0}\right)\left(T_{0} \mid\right.$ in Eq. (136). For this reason, we work with the $\left.\mid \chi_{\sigma}\right)$ basis (112) in the general case.

a. Full spin-rotation symmetry, $B=0$. At zero magnetic field, all terms in the Liouvillian must be rank-0 ITSOs (scalars) with respect to spin rotations, i.e., the terms $\left.\mid S_{0}\right)\left(S_{0} \mid\right.$ and $\left.\mid S_{ \pm}\right)\left(S_{ \pm} \mid\right.$must have the same coefficients $E_{+}=E_{-}=\xi_{S S}$ and the coefficients of the rank-1 and -2 spin ITSOs must vanish, i.e., in the $\chi$ subspace $\left.\mid \phi)=\phi_{T} \mid T_{0}\right)$ and $\left.|\psi\rangle=\psi_{T} \mid T_{0}\right)$ with real $\phi_{T}$ and $\psi_{T}$ at $z=i 0$ [cf. Eq. (144)]:

$$
\begin{aligned}
i \bar{L}_{\Lambda}= & \left.\cdots+\phi_{T} \mid Z_{R}\right)\left(T_{0}\left|+\psi_{T}\right| T_{0}\right)\left(Z_{L} \mid\right. \\
& \left.+M_{+} \mid T_{+}\right)\left(T_{+}\left|+M_{-}\right| T_{-}\right)\left(T_{-}\left|+\xi_{T T}\right| T_{0}\right)\left(T_{0} \mid\right. \\
& +\xi_{S S}\left(\mid S_{+}\right)\left(S_{+}|+| S_{-}\right)\left(S_{-}|+| S_{0}\right)\left(S_{0} \mid\right)+\cdots .
\end{aligned}
$$

See Table II, where the ITSOs are listed. The expression for the stationary state $\rho$ obtained using Eq. (151) is then independent of $\xi_{S S}$ and is therefore explicitly invariant under spin rotations, as required:

$$
\left.\left.\left.\rho=-\frac{1}{2} \psi_{T} \mid T_{0}\right)+\frac{1}{2} \mid Z_{L}\right)-\frac{\zeta-\phi_{T} \psi_{T} / \xi_{T T}}{8 \Gamma} \mid Z_{R}\right) .
$$

b. Full charge-rotation symmetry, $\epsilon=-U / 2$ and $\mu_{L}=$ $\mu_{R}$. At the particle-hole symmetric point, a similar reduction must take place: here $\left.\left.M_{+}=M_{-}=\xi_{T T}, \mid \phi\right)=\phi_{S} \mid S_{0}\right)$, and $\left.|\psi\rangle=\psi_{S} \mid S_{0}\right)$ :

$$
\begin{aligned}
i \bar{L}_{\Lambda}= & \left.\cdots+\phi_{S} \mid Z_{R}\right)\left(S_{0}\left|+\psi_{S}\right| S_{0}\right)\left(Z_{L} \mid\right. \\
& +\xi_{T T}\left(\mid T_{+}\right)\left(T_{+}|+| T_{-}\right)\left(T_{-}|+| T_{0}\right)\left(T_{0} \mid\right) \\
& \left.+E_{+} \mid S_{+}\right)\left(S_{+}\left|+E_{-}\right| S_{-}\right)\left(S_{-}\left|+\xi_{S S}\right| S_{0}\right)\left(S_{0} \mid\right.
\end{aligned}
$$

and the stationary state is explicitly invariant under charge rotations:

$$
\left.\left.\left.\rho=-\frac{1}{2} \psi_{S} \mid S_{0}\right)+\frac{1}{2} \mid Z_{L}\right)-\frac{\zeta-\phi_{S} \psi_{S} / \xi_{S S}}{8 \Gamma} \mid Z_{R}\right) .
$$

\section{E. Current superoperator and its irreducible self-energy}

Our main objective is to calculate the stationary current that flows through the QD. Having set up the perturbation theory formalism for the density operator, the expression for the average current can now be compactly derived. Moreover, we give a general proof that in general at zero bias the current vanishes, as it should, independent of the way the self-energy is calculated. 
The current flowing out of reservoir $r=L$ or $R$ is obtained using Heisenberg operators (with index $\mathrm{H}$ ): $I_{\mathrm{H}}^{r}=$ $-\frac{d}{d t} n_{\mathrm{H}}^{r}=-i\left[H^{\mathrm{tot}}, n_{\mathrm{H}}^{r}\right]_{-}=-i\left[V_{\mathrm{H}}^{r}, n_{\mathrm{H}}^{r}\right]_{-}$. Note that there is no summation over the electrode index $r$ [cf. Eq. (10)]. The expectation value of the Schrödinger-picture current operator

$$
I^{r}=-i\left[V^{r}, n^{r}\right]_{-}
$$

can be expressed in superoperators using the cyclic invariance of the total system trace:

$$
\begin{aligned}
\left\langle I^{r}\right\rangle(t) & =\operatorname{Tr}_{D \mathrm{R}}\left(I^{r} \rho^{\mathrm{tot}}(t)\right) \\
& =-i \operatorname{Tr}_{D} \operatorname{Tr}\left(L^{I^{r}} e^{-i L^{\mathrm{tot}}\left(t-t_{0}\right)} \rho\left(t_{0}\right) \rho^{\mathrm{R}}\right) .
\end{aligned}
$$

We note that observable averages involve anticommutators of the corresponding operator (see, e.g., Ref. 44):

$$
L^{I^{r}}=\frac{i}{2}\left[I^{r}, \bullet\right]_{+} \cdot
$$

This is in contrast to time-evolution superoperators, which involve commutators of the Hamiltonian operator. This difference is exploited below. If one uses Eq. (174) in Eq. (175), the evaluation of the reservoir trace is unnecessarily complicated since Eq. (174) involves two operators acting on the reservoir, $V^{r}$ and $n^{r}$. Here, we proceed differently: we first use that the tunneling through junction $r$ conserves the particle number of the dot and the reservoir $r$, i.e., $\left[n^{r}+n, V^{r}\right]_{-}=0$, to eliminate one electrode operator:

$$
I^{r}=i\left[V^{r}, n\right]_{-}=-i\left[n, V^{r}\right]_{-} .
$$

Then, using the identity $\left[[A, B]_{-}, \bullet\right]_{+}=\left[A,[B, \bullet]_{-}\right]_{+}-$ $\left[B,[A, \bullet]_{+}\right]_{-}$the current superoperator anticommutator can be decomposed as

$$
L^{I^{r}}=\frac{1}{2}\left(L^{n+} L^{V, r}-L^{V, r} L^{n+}\right) .
$$

Here, we introduced the anticommutator superoperator for the particle number [cf. Eq. (43)]

$$
L^{n+}=[n, \bullet]_{+},
$$

and decomposed the tunneling interactions into the junction contributions $L^{V}=\sum_{r} L^{V, r}$. Importantly, the last term of Eq. (178) is irrelevant when inserted into Eq. (175) since $\operatorname{Tr}_{D} \operatorname{Tr}_{\mathrm{R}} L^{V, r} \bullet=0$ due to the commutator form of $L^{V, r}$. We obtain

$$
\left\langle I^{r}\right\rangle(t)=-i \frac{1}{2} \underset{D}{\operatorname{Tr}} L^{n+}\left(\underset{\mathrm{R}}{\operatorname{Tr}} L^{V, r} \rho^{\mathrm{tot}}(t)\right) .
$$

Integrating out of the reservoirs and collecting terms into irreducible blocks (cf. Sec. II B3), one now obtains

$$
\begin{aligned}
\left\langle I^{r}\right\rangle(z) & =\frac{1}{2} \operatorname{Tr}_{D} L^{n+} \Sigma^{r}(z) \frac{1}{z-L(z)} \rho\left(t_{0}\right) \\
& =-\frac{1}{2} i \operatorname{Tr}_{D} L^{n+} \Sigma^{r}(z) \rho(z),
\end{aligned}
$$

where $\Sigma^{r}$ is just that part of the irreducible self-energy $\Sigma$ for which the latest (leftmost) vertex is associated with reservoir $r$. We can thus decompose

$$
\Sigma(z)=\sum_{r} \Sigma^{r}(z)
$$

For the stationary current $\left\langle I^{r}\right\rangle=\lim _{t-t_{0} \rightarrow \infty}\left\langle I^{r}\right\rangle(t)=$ $\lim _{z \rightarrow i 0}-i z\left\langle I^{r}\right\rangle(z)$, we then obtain the central result of this section:

$$
\left\langle I^{r}\right\rangle=-i \frac{1}{2} \operatorname{Tr}_{D} L^{n+} \Sigma^{r}(i 0) \rho,
$$

where $\rho$ is the stationary density operator [cf. Eq. (151)].

The first advantage of Eq. (183) is that it allows one to explicitly see that the current is always zero at zero bias. For any number of electrodes, at zero voltage bias all electrochemical potentials are equal, $\mu_{r}=0$, implying that all partial self-energies $\Sigma^{r}$ are proportional to the total self-energy:

$$
\Sigma^{r} \propto \frac{\Gamma_{r}}{\sum_{r} \Gamma_{r}} \Sigma(i 0) .
$$

Next, we add $L$ to $\Sigma(i 0)$ in Eq. (183) without changing its value since by local charge conservation [Eq. (23)], $\operatorname{Tr}_{D} L^{n+} L \bullet=$ $\operatorname{Tr}_{D} n[H, \bullet]_{-}=\operatorname{Tr}_{D}[n, H]_{-} \bullet=0$ when acting on any dot operator. We can thus express the stationary, zero-bias current in terms of the effective Liouvillian $L(z)=L+\Sigma(z)$ and directly see that it must vanish,

$$
\left\langle I^{r}\right\rangle \propto-i \operatorname{Tr}_{D} L^{n+} L(i 0) \rho=0
$$

since the stationary state $\rho$ is the zero eigensupervector of $L(i 0)$ [cf. Eq. (37)]. We note, however, that the relation (184) relies on the assumption of reservoir-frequency-independent spectral densities $\Gamma_{r}(\omega)=\Gamma_{r}$, which we make throughout this paper. Apart from that, the above proof holds no matter what approximations one makes for the calculation of the self-energy $\Sigma(i 0)$ as long as all reservoirs are treated in the same way. This applies to perturbation theory up to any finite order, as well as to the RT-RG approach that we set up in Sec. III.

A second advantage of Eq. (183) is that we can directly relate the current to just a few supermatrix elements of the zerofrequency effective Liouvillian $L(i 0)$ in the basis introduced in Eq. (136). The dot trace combined with the action of $L^{n+}$ can be expressed in the dual supervectors of (103) and (108):

$$
\frac{1}{2} \underset{D}{\operatorname{Tr}}\left(L^{n+} \bullet\right)=\operatorname{Tr}_{D}(n \bullet)=\left(\sqrt { 2 } \left(T_{0} \mid+2\left(Z_{L} \mid\right) \bullet .\right.\right.
$$

Equation (85) implies probability conservation, Eq. (69), but also, more strongly, that $\left(Z_{L} \mid \Sigma^{r}(i 0) \bullet=2 \operatorname{Tr}_{D} \Sigma^{r}(i 0) \bullet=0\right.$ for fixed $r$. From this, we obtain

$$
\left\langle I^{r}\right\rangle=-\sqrt{2} i\left(T_{0}\left|\Sigma^{r}\right| \rho\right) .
$$

Clearly, the partial self-energy $\Sigma^{r}$ has the same general form as Eq. (136) and we distinguish its parameters (except $\Gamma_{r}$ ) by an additional reservoir superscript $r$ :

$$
\begin{aligned}
i \Sigma^{r}= & \left.2 \Gamma_{r} \mid Z_{R}\right)\left(Z_{R}\left|+\zeta^{r}\right| Z_{R}\right)\left(Z_{L}|+| Z_{R}\right)\left(\overrightarrow{\phi^{r}}|+| \overrightarrow{\psi^{r}}\right)\left(Z_{L} \mid\right. \\
& +\xi^{r}+\sum_{\sigma}\left[M_{\sigma}^{r} \mid T_{\sigma}\right)\left(T_{\sigma}\left|+E_{\sigma}^{r}\right| S_{\sigma}\right)\left(S_{\sigma} \mid+\sum_{\eta} F_{\eta, \sigma}^{r}\right] .
\end{aligned}
$$

Inserting this form and the explicit expression for the stationary state Eq. (151) into Eq. (187), we obtain the final explicit expression for the average stationary current in terms of the 
self-energy expansion coefficients:

$$
\left\langle I^{r}\right\rangle=\frac{1}{\sqrt{2}}\left[\left(T_{0} \mid \vec{\psi}^{r}\right)-\left(T_{0}\left|\xi^{r} \xi^{-1}\right| \vec{\psi}\right)\right] .
$$

We emphasize that this equation is exact, given that the coefficients $\psi_{\sigma}^{r}$ and $\xi^{r}$ of the partial self-energies $\Sigma^{r}$ are known, from which $\psi_{\sigma}=\sum_{r} \psi_{\sigma}^{r}$ and $\xi=\sum_{r} \xi^{r}$ also follow. We can thus calculate the current easily if we perform all self-energy calculations separately for each fixed value of the reservoir index $r$ at the latest (leftmost) vertex and sum over $r$ to obtain Eq. (182). Finally, we note that using Eq. (189), one can check explicitly that if one imposes particle-hole symmetry on the expansion coefficients in Eq. (188), then the current (187) vanishes: one finds that $\mid \psi^{r}$ ) and $\xi^{r}$ have no components involving $\left.\mid T_{0}\right)$. This is in agreement with the result (185) obtained above making explicit use of $\mu_{L}=\mu_{R}$.

\section{REAL-TIME RENORMALIZATION GROUP}

In this section, we set up the real-time renormalization group (RT-RG) calculation of the effective Liouvillian $L(z)$. It is based on the perturbative expansion of the "finitetemperature" self-energy $\bar{\Sigma}$ in the causal representation introduced in the previous section (cf. Sec. II B4). The procedure is to calculate $L(z)$ by introducing a RG flow of the "infinitetemperature" Liouvillian $\bar{L}$ and the vertex $\bar{G}$ as function of a decreasing energy scale cutoff $\Lambda$, with the initial conditions given by $\bar{L}=\tilde{\Sigma}+L$ (thereby including the vertex of the type $\tilde{G}$ ) and $\bar{G}$, the vertex of the perturbation theory. There are a number of motivations for performing such a RG treatment of the perturbative series for $\bar{\Sigma}$.

(i) First of all, treating $\bar{\Sigma}$ perturbatively in $\bar{G}$, while infinite orders of $\tilde{G}$ have already been resummed into $\tilde{\Sigma}$, would amount to an inconsistent treatment. We note that even in the noninteracting case $U=0$, we already need to do a renormalized perturbation theory (79) up to two-loop terms to recover the exact result for all quantities (i.e., not just the current). For strong interaction $U$, higher-order corrections in $\bar{G}$ become increasingly important: at finite and especially at low $T$ the strong interaction $U$ leads to different lifetime broadening for single- (SET) and two-electron inelastic cotunneling (ICT) excitations, with a nontrivial voltage dependence. This is not described by $\tilde{\Sigma}$ : it leads to a broadening of the various excitations of the QD that is energy independent and $\sim \Gamma$. The nontrivial, energy-dependent corrections due to quantum fluctuations contained in the high-order contributions to $\bar{\Sigma}(z)$ are required.

(ii) Second, although the bare perturbation theory breaks down at these resonances as $T \rightarrow 0$, in the renormalized perturbation theory (79), the low-energy cutoff $T$ is replaced by the imaginary parts $\sim \Gamma$ in $\bar{L}$. Still, the resonance due to the Kondo effect causes even the renormalized perturbation theory to break down and three-loop corrections result in the enhancement of Kondo exchange processes. These have been studied extensively using the RT-RG based on an effective Kondo model obtained by a Schrieffer-Wolff transformation from the Anderson model. ${ }^{27}$ In the regime of large applied bias voltage and/or magnetic field, such three-loop corrections can be neglected due to the dephasing of the Kondo correlations. This is the regime of interest in this work for which we expect the one-plus-two-loop RG approach to give a good first approximation that deserves to be studied.

(iii) In order to study the Kondo effect in the Anderson model beyond Ref. 27, at least a three-loop treatment is necessary. The renormalization of the one- and two-loop terms that we study here will then couple to the three-loop terms and still play an important role. Therefore, our study of the two-loop RG provides an important starting point for such a much more involved study, which in particular can address the low bias and low magnetic field regime.

\section{A. Flow of Keldysh contractions: Continuous RG}

In general, RG approaches to transport aim to eliminate the effect of reservoirs states, starting at high energies, by incorporating it into a redefinition of the system parameters. Typically, one eliminates the states themselves. Here, in contrast, we successively suppress the occupations of the states using a RG procedure while keeping the states. Before we specify the details and advantages of this cutoff scheme, we first outline the main idea of the functional renormalization group approach when applied to the real-time perturbation series (79). By our causal reformulation of the perturbation theory (cf. Sec. II B2), it is clear that all the information about the occupations of the reservoir states is contained in the Keldysh components of the correlation functions, i.e., in the $\bar{\gamma}$ contraction. Therefore, we introduce a cutoff-dependent contraction function $\bar{\gamma}_{\Lambda}$ that monotonously flows from the initial, full contraction function $\left.\bar{\gamma}_{\Lambda}\right|_{\Lambda=\infty}=\bar{\gamma}$ given by Eq. (64) to the trivial final function $\left.\bar{\gamma}_{\Lambda}\right|_{\Lambda=0}=0$ where all occupations are suppressed. During this flow, we demand that the effective Liouvillian remains invariant: for every value of the cutoff parameter $\Lambda$,

$$
\begin{aligned}
L(z) & =\bar{L}+\bar{\Sigma}(\{\bar{\gamma}, \bar{L}, \bar{G}\}) \\
& =\bar{L}_{\Lambda}+\bar{\Sigma}\left(\left\{\bar{\gamma}_{\Lambda}, \bar{L}_{\Lambda}, \bar{G}_{\Lambda}\right\}\right) \\
& =\left.\bar{L}_{\Lambda}\right|_{\Lambda=0} .
\end{aligned}
$$

Thus, $L(z)$ has the same functional dependence on the contractions $\gamma_{\Lambda}$, the Liouvillian $\bar{L}_{\Lambda}$, and vertices $\bar{G}_{\Lambda}$. The latter two now acquire a $\Lambda$ dependence to maintain invariance. The same diagram rules are thus valid for any value of $\Lambda$. As a result, at the end of the flow where $\left.\bar{\gamma}_{\Lambda}\right|_{\Lambda=0}=0$, the effective Liouvillian is given simply by $\left.\bar{L}_{\Lambda}\right|_{\Lambda=0}$. The information about the reservoir degrees of freedom, previously incorporated into the self-energy $\bar{\Sigma}$, has now been incorporated fully into the dot Liouvillian [cf. Eq. (192)].

The $R G$ flow is generated by making an infinitesimal change $d \Lambda$ of the cutoff, resulting in a infinitesimal change $d \bar{\gamma}_{\Lambda} \approx\left(d \bar{\gamma}_{\Lambda} / d \Lambda\right) d \Lambda$ of the Keldysh contraction function. In the perturbation series at scale $\Lambda$, one splits up the contraction function as $\bar{\gamma}_{\Lambda}=\bar{\gamma}_{\Lambda-d \Lambda}+d \bar{\gamma}_{\Lambda}$ and collects all terms in a perturbation series of the same form but containing only $\bar{\gamma}_{\Lambda-d \Lambda}$ contractions. In this process, the terms containing one infinitesimal contraction $d \bar{\gamma}_{\Lambda}$ can be identified with renormalizations $d \bar{L}$ of the Liouvillian, $d \bar{G}$ of the vertices, and newly generated higher-order vertices with more than one leg. The process is illustrated in Fig. 4. The Liouvillian $\bar{L}_{\Lambda-d \Lambda}=\bar{L}_{\Lambda}-d \bar{L}_{\Lambda}$, and vertices $\bar{G}_{\Lambda-d \Lambda}=\bar{G}_{\Lambda}-d \bar{G}_{\Lambda}$ of 


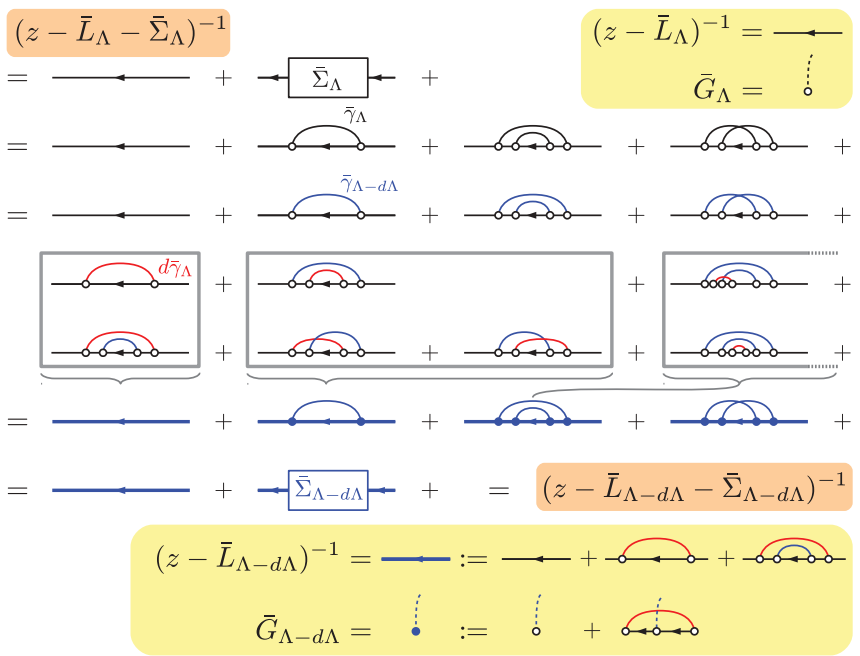

FIG. 4. (Color online) Renormalization group transformation with an infinitesimal change $d \Lambda>0$ of the flow parameter $\Lambda \rightarrow$ $\Lambda-d \Lambda$. The physical restriction is that the effective Liouvillian $L(z)=\bar{L}_{\Lambda}+\bar{\Sigma}_{\Lambda}$ or, equivalently, the density operator propagator $[z-L(z)]^{-1}$, remains unchanged. In lines 1 and 2 we start from the perturbation theory at scale $\Lambda$ and split up the contraction function $\gamma_{\Lambda}$ (black curved lines) into the contraction function with reduced flow parameter $\left(\gamma_{\Lambda-d \Lambda}\right.$, blue curved line), which should appear in the renormalized perturbation series and change the contraction function $\left(-d \gamma_{\Lambda}\right.$, red curve line). Next, in lines 3-5, the latter terms of linear order in $d \gamma_{\Lambda}$ are collected into one- and two-loop renormalizations $d \bar{L}_{\Lambda}$ of the Liouvillian in the resolvents and one-loop renormalization $d \bar{G}_{\Lambda}$ of the vertices. Finally, the perturbation series is rewritten in terms of new Liouvillian $\bar{L}_{\Lambda-d \Lambda}$ and vertices $\bar{G}_{\Lambda-d \Lambda}$ defined on the new scale $\Lambda-d \Lambda$ (indicated by blue). This transformation is exact if one also accounts for the generation of higher-order vertices (Ref. 26), which we, however, neglect here (they are not drawn). We do account for the renormalization of the original vertices, i.e., of single-charge fluctuations.

the new perturbation series are then all defined for the new, lower cutoff scale $\Lambda-d \Lambda$. We obtain differential equations for these quantities describing their renormalization as one continuously reduces the cutoff $\Lambda$. These are the real-time renormalization group (RT-RG) equations.

A key requirement in setting up this continuous $R G$ is that for any $\Lambda$, the zero eigenvector of $\bar{L}^{\Lambda}$ does not appear in the resolvents $\left(z+X-\bar{L}^{\Lambda}\right)^{-1}$, to avoid divergences as function of the frequencies. The RG thus has to be formulated such that the property (85) of the vertices in the causal representation is preserved. This can be shown to be the case [see Eq. (212)].

The final key point is the choice of a cutoff-dependent distribution function in the contraction $\bar{\gamma}$. The numerical integration of the RG equations is more stable when we introduce a contraction function with a cutoff on the imaginary frequency axis,

$$
\bar{\gamma}_{12, \Lambda}(\eta \omega)=\delta_{1 \overline{2}} \frac{\Gamma}{\pi} T \sum_{l=0}^{\infty} \frac{\Theta_{T}\left(\Lambda-\left|\omega^{l}\right|\right)}{\eta \omega-\bar{\mu}_{r}-i \omega^{l}},
$$

through the function

$$
\Theta_{T}(\omega)= \begin{cases}\Theta(\omega), & |\omega|>\pi T, \\ \frac{1}{2}+\frac{\omega}{2 \pi T}, & |\omega|<\pi T\end{cases}
$$

where $\Theta(\omega)$ is the step function and

$$
\omega^{l}=(2 l+1) \pi T
$$

is the $l$ th Matsubara frequency $(l=0,1,2, \ldots)$. In the limit $\Lambda \rightarrow+\infty$, we recover the partial fraction expansion of the meromorphic function $(\Gamma / 2 \pi) \tanh (\omega / 2 T)$ as required. Imposing this cutoff in Matsubara space leads to a suppression of the tails of $\bar{\gamma}^{\Lambda}(\eta \omega)$ on the real frequency axis as $\Lambda \rightarrow 0$ rather than sharp truncation above frequency $\Lambda$. This implements the suppression of contributions from states above energy scale $\Lambda$. From here on, we will consider the zero-temperature limit $T \rightarrow 0$ for which the contraction function (193) reduces to the simple form

$$
\bar{\gamma}_{12, \Lambda}(\eta \omega)=\delta_{1 \overline{2}} \frac{\Gamma}{\pi} \int_{-\Lambda}^{\Lambda} d \omega^{\prime} \frac{1}{\eta \omega-\bar{\mu}_{r}-i \omega^{\prime}} .
$$

See Ref. 26 for a detailed discussion. ${ }^{59}$

\section{B. RG in frequency space}

\section{Nonequilibrium Matsubara representation and frequency dependence}

To formulate the RG equations, we need a more compact notation for the various frequencies. Since the contraction functions $\bar{\gamma}^{\Lambda}$ depend on $\eta \omega=x-\eta \mu$, we reexpress the reservoir energies in the resolvents in $\bar{\Sigma}$ [Eq. (79)] in explicit calculations as

$$
E-X_{i}=E-x_{1 \ldots n}=E_{1 \ldots n}-\bar{\omega}_{1 \ldots n} .
$$

Here, $1, \ldots, n$ are the multi-indices of the contractions going over diagram segment $i$. The frequencies are now taken relative to the electrochemical potentials and we write their sums as repeated multi-indices: for $k=\eta_{k}, \sigma_{k}, r_{k}, \omega_{k}$,

$$
\begin{gathered}
x_{k}=\bar{\omega}_{k}+\bar{\mu}_{k}, \quad x_{1 \ldots n}=x_{1}+\cdots+x_{n}, \\
\bar{\omega}_{k}=\eta_{k} \omega_{k}, \quad \bar{\omega}_{1 \ldots n}=\bar{\omega}_{1}+\cdots+\bar{\omega}_{n}, \\
\bar{\mu}_{k}=\eta_{k} \mu_{k}, \quad \bar{\mu}_{1 \ldots n}=\bar{\mu}_{1}+\ldots+\bar{\mu}_{n} .
\end{gathered}
$$

Similarly, we express the dot energies relative to these chemical potentials as

$$
E_{1 \ldots n}=E-\sum_{i=1 \ldots n} \bar{\mu}_{i} .
$$

A key advantage of the cutoff parametrization (193) is that it allows us to analytically perform all the integrations over the reservoir frequencies $\bar{\omega}$ in Eq. (79) by closing each integration contour in the complex lower half-plane and by using the residual theorem. As a result, we can replace all integrations over real frequencies in the resolvents by summations over Matsubara frequencies lying in the lower half-plane: Eq. (197) becomes

$$
E_{1 \ldots n}+i \omega_{1 \ldots n}
$$

For finite $T$, the $\omega_{k}$ sum runs over the positive discrete frequency values $\left(2 l_{k}+1\right) \pi T, l_{k}=0,1,2, \ldots$, which turns into an integral over positive $\omega_{k}$ for $T=0 .{ }^{60}$ As always, we do not explicitly indicate this integral. 


\section{2. $R G$ equations}

During the RG flow, the Liouvillian develops a nontrivial dependence on both the real energy $E$ of the QD and on $i \omega$, the sum of the imaginary frequencies of all the reservoir contractions that pass over the propagator in a diagram. This is illustrated in Fig. 5(a). We separately keep track of these frequency dependencies by writing

$$
\begin{gathered}
\bar{L}_{\Lambda}(E, \omega):=\bar{L}_{\Lambda}(E+i \omega), \\
\Pi_{\Lambda}(E, \omega):=\frac{1}{E+i \omega-\bar{L}_{\Lambda}(E, \omega)} .
\end{gathered}
$$

The renormalization of the vertex $\bar{G}_{1}$ introduces similar dependencies and an additional dependence on the reservoir frequency $\omega_{1}$ of the vertex "leg," i.e., the contraction connecting it to another vertex [see Fig. 5(b)]:

$$
\bar{G}_{1, \Lambda}\left(E, \omega, \omega_{1}\right):=\bar{G}_{1, \Lambda}\left(E+i \omega, i \omega_{1}\right) .
$$

The formally exact, infinite hierarchy of RT-RG equations was derived in general form in Ref. 26. Here, we restrict ourselves to the one- and two-loop order approximation for the Liouvillian and the limit of $T \rightarrow 0$ :

$$
\begin{aligned}
\frac{d \bar{L}(E, \omega)}{d \Lambda}= & i \frac{\Gamma}{\pi} \bar{G}_{1}(E, \omega, \Lambda) \Pi\left(E_{1}, \omega+\Lambda\right) \bar{G}_{\overline{1}}\left(E_{1}, \omega+\Lambda,-\Lambda\right)+\frac{\Gamma^{2}}{\pi^{2}} \bar{G}_{1}(E, \omega, \Lambda) \Pi\left(E_{1}, \omega+\Lambda\right) \bar{G}_{2}\left(E_{1}, \omega+\Lambda, \omega_{2}\right) \\
& \times \Pi\left(E_{12}, \omega+\Lambda+\omega_{2}\right) \bar{G}_{\overline{2}}\left(E_{12}, \omega+\Lambda+\omega_{2},-\omega_{2}\right) \Pi\left(E_{1}, \omega+\Lambda\right) \bar{G}_{\overline{1}}\left(E_{1}, \omega+\Lambda,-\Lambda\right) .
\end{aligned}
$$

This approximation requires that one also accounts for the renormalization of vertices to one-loop order:

$$
\frac{d \bar{G}_{1}\left(E, \omega, \omega_{1}\right)}{d \Lambda}=-i \frac{\Gamma}{\pi} \bar{G}_{2}(E, \omega, \Lambda) \Pi\left(E_{2}, \omega+\Lambda\right) \bar{G}_{1}\left(E_{2}, \omega+\Lambda, \omega_{1}\right) \Pi\left(E_{12}, \omega+\omega_{1}+\Lambda\right) \bar{G}_{\overline{2}}\left(E_{12}, \omega+\omega_{1}+\Lambda,-\Lambda\right) .
$$

Here and in the following, we leave implicit both the $\Lambda$ dependence of the renormalized $\bar{L}$ and $\bar{G}$ as well as the summation/integration over all internal indices. ${ }^{61}$

By construction, the self-energy $\bar{\Sigma}(z)$ is obtained by directly integrating Eq. (206):

$$
\bar{\Sigma}(E+i \omega)=L(E+i \omega)-\bar{L}=\int_{\infty}^{0} d \Lambda \frac{d \bar{L}_{\Lambda}}{d \Lambda}(E, \omega) .
$$

(a)

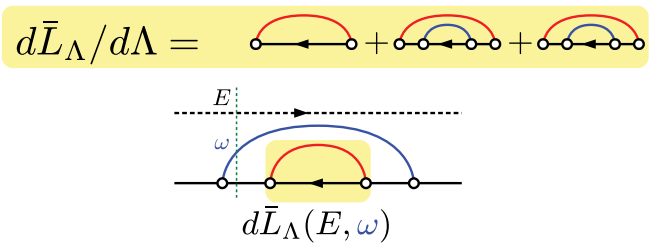

(b)

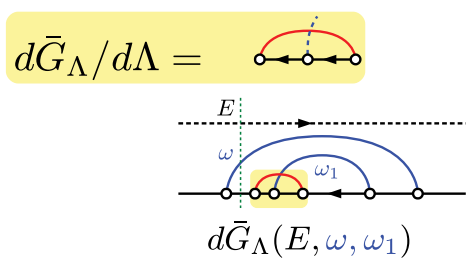

FIG. 5. (Color online) RG equations and frequency dependence generated by the renormalization transformation of the diagrammatic perturbation theory in Fig. 4 (using the same red and blue colors). (a) Liouvillian renormalization by one- and two-loop corrections. The example diagram illustrates that the correction depends on the external frequency $E$ of the diagram (Laplace variable) and on the sum of the reservoir frequencies $\omega$ running over the $d \bar{L} / d \Lambda$ block (marked yellow). These frequencies are read off at the vertical cut (green dashed line) to the left of this block. (b) Vertex renormalization by one-loop corrections. The example diagram illustrates that the vertex correction depends additionally on frequency $\omega_{1}$ of the vertex leg.
For the transport current we need the reservoir-resolved parts $\bar{\Sigma}^{r}(z)$ of this self-energy [cf. Eq. (182)], which are obtained in the same way: for $r=L, R$,

$$
\bar{\Sigma}^{r}(E+i \omega)=L^{r}(E+i \omega)-\bar{L}^{r}=\int_{\infty}^{0} d \Lambda \frac{d \bar{L}_{\Lambda}^{r}}{d \Lambda}(E, \omega),
$$

where $\left.\bar{L}_{\Lambda}^{r}\right|_{\Lambda=\infty}=\bar{L}^{r}:=\tilde{\Sigma}^{r}$ is given by Eq. (71). The RG equations determining $\bar{L}_{\Lambda}^{r}$ and its associated vertex $G^{r}$ have the same structure as the RG equations (206) and (207) and are simply obtained from the latter ${ }^{26}$ by providing the leftmost vertex with a superscript $r$ on the right-hand sides of Eqs. (206) and (207) and on the left-hand side of Eq. (207). We emphasize that $\sum_{r} \bar{L}^{r}=\tilde{\Sigma}=\bar{L}-L$, whereas $\left.\sum_{r} \bar{L}_{\Lambda}^{r}\right|_{\Lambda=0}=$ $\Sigma(z)=L(z)-L{ }^{6}{ }^{6}$

The frequency dependence in the RG equations (206) and (207) in the one- and two-loop approximation needs to be carefully discussed. Before we turn to this in Secs. III C and IIID, we discuss some important general properties of the RG equations in Sec. III B3 and their implications for the fermionic eigenvalues in Sec. III B4. Using these results, we can decouple some of the RG equations (see Sec. III C) and show that in the one-loop approximation, we already obtain the exact solution for the current in the limit $U=0$, even though in this limit two-loop corrections are nonzero (see Sec. III C3).

\section{Exact eigenvectors}

The exact properties derived in Sec. II C5a follow from the causal structure of the perturbation series (79). Since the RG equations (206) and (207) represent nothing but a reorganization of the terms in that expansion (as explicitly shown in Ref. 26), we expect that they preserve these causal structure properties. We now show that this is indeed the case. Indeed, an exact left and right eigensupervector of the 
renormalized $\bar{L}$ is given by

$$
\begin{gathered}
\left(Z_{L} \mid \bar{L}(E, \omega)=0,\right. \\
\left.\left.\bar{L}(E, \omega) \mid Z_{R}\right)=-i 4 \Gamma \mid Z_{R}\right),
\end{gathered}
$$

respectively. Since at $\Lambda=\infty$ we have the initial condition $\left(Z_{L} \mid \bar{L}=0\right.$ and $\left.\left.\bar{L} \mid Z_{R}\right)=-i 4 \Gamma \mid Z_{R}\right)$, we only need to show that $\left(Z_{L}\left|\frac{d}{d \Lambda} \bar{L}=\frac{d}{d \Lambda} \bar{L}\right| Z_{R}\right)=0$, respectively, to prove Eqs. (210) and (211). We note that since Eq. (208) is an exact relation, Eqs. (210) and (211) must hold for the exact, infinite hierarchy of RT-RG equations (i.e., including all higher vertices generated during the RG flow that we neglect here). In our two-loop approximation, this relation directly follows by letting Eq. (206) act on these vectors and using

$$
\begin{aligned}
& \left(Z_{L} \mid \bar{G}\left(E, \omega, \omega_{1}\right)=0,\right. \\
& \left.\bar{G}\left(E, \omega, \omega_{1}\right) \mid Z_{R}\right)=0 .
\end{aligned}
$$

Equations (212) and (213) follow by assuming that they hold for a given $\Lambda$, and by $\left(Z_{L}\left|\frac{d}{d \Lambda} \bar{G}=\frac{d}{d \Lambda} \bar{G}\right| Z_{R}\right)=0$, obtained acting with Eq. (207) on these vectors. Since Eqs. (212) and (213) hold initially for $\Lambda=\infty$, the result follows for any $\Lambda, E, \omega$, and $\omega_{1}$.

Similarly, we now show that for any $\Lambda, E, \omega$, and $\omega_{1}$,

$$
\begin{aligned}
& \left.\frac{d}{d \Lambda} \bar{L}(E, \omega) \mid \alpha_{\eta \sigma}^{-}\right)=0, \\
& \left(\alpha_{\eta \sigma}^{+} \mid \frac{d}{d \Lambda} \bar{L}(E, \omega)=0 .\right.
\end{aligned}
$$

In our two-loop approximation for $d \bar{L} / d \Lambda$, this follows from the property of the renormalized one-leg vertices

$$
\begin{gathered}
\left.\left.\bar{G}\left(E, \omega, \omega_{1}\right) \mid \alpha_{\eta \sigma}^{-}\right) \propto \mid Z_{R}\right) \text { or } 0, \\
\left(\alpha_{\eta \sigma}^{+} \mid \bar{G}\left(E, \omega, \omega_{1}\right) \propto\left(Z_{L} \mid \text { or } 0 .\right.\right.
\end{gathered}
$$

The proof of Eqs. (122) and (121) can be extended to the renormalized vertices as follows. We start by observing that the right-hand sides of the RG equations (206) and (207) have the same structure as $\bar{\Sigma}(z)$ [cf. Eq. (79)]. Assuming that Eq. (216) holds for a given scale $\Lambda$, the RG equation (207) implies that it is preserved under the flow: $\left(\alpha_{\eta \sigma}^{+} \mid \frac{d}{d \Lambda} \bar{G}=\right.$ $\left.\frac{d}{d \Lambda} \bar{G} \mid \alpha_{\eta \sigma}^{-}\right)=0$. Here, we used that by Eqs. (210) and (211), both $\left.\mid Z_{R}\right)$ and $\left(Z_{L} \mid\right.$ are eigensupervectors of the renormalized $\bar{L}$ for all $\Lambda, E, \omega, \omega_{1}$. Since Eqs. (216) and (217) hold initially for $\Lambda=\infty$, this then implies it holds for all $\Lambda$. From this, Eqs. (214) and (215) follow directly. The above proofs are readily extended to the infinite hierarchy of exact RT-RG equations for vertices with multiple legs, confirming that Eqs. (210), (211), (215), and (214) hold exactly (and not just in our two-loop approximation).

\section{Fermionic excitations}

a. Fermionic eigenvalues. We can now pick up the discussion of Sec. II D. Since the supermatrix structure of $d \bar{L}_{\Lambda} / d \Lambda$ in the fermionic sector is preserved under the RG flow and is the same as that of $\bar{\Sigma}(z)$, we can now directly relate the coefficient $\Delta F_{\eta \sigma}^{-+}$introduced in Sec. II D3 using Eq. (208):

$$
\Delta F_{\eta \sigma}^{-+}(E+i \omega)=\int_{\infty}^{0} d \Lambda \frac{d F_{\eta \sigma, \Lambda}^{-+}}{d \Lambda}(E+i \omega) .
$$

This coefficient determines the fermionic excitations at arbitrary complex frequency as given by Eq. (163) for the exact $L(z)$ when the infinite hierarchy of RG equations is used to compute the right-hand side. We see that the $\Lambda$ dependent coefficient $F_{\eta \sigma, \Lambda}^{-+}$of $\bar{L}_{\Lambda}$ interpolates between the infinite-temperature limit, where $\Delta F_{\eta \sigma}^{-+}=0$, and the exact value $\Delta F_{\eta \sigma}^{-+}$of $\Sigma(z)$ through Eq. (218) as was anticipated in Sec. II D. All renormalization effects enter into the fermionic excitations through the renormalization of the four complex coefficient $F_{\eta, \sigma}^{-+}$of $\bar{L}_{\Lambda}$. During this flow, the qualitative features of these excitations, discussed in Fig. 3, may change. During the continuous RG, the complex parameters $\Delta F_{\eta, \sigma}^{-,+}$ will grow from zero and modify both real and imaginary parts in Eq. (163). This happens only for the interacting Anderson model $U \neq 0$ since $U$ multiplies these coefficients in Eq. (163). This flow may include bifurcations as function of the flow parameter $\Lambda$, but for large enough $U \gg 2 \Gamma$ the excitation energies (real parts) remain nondegenerate. However, the general result (168) shows that during this nontrivial flow, the average of the complex eigenvalues stays fixed for all frequencies. We conclude generally that the fermionic excitation energies and decay rates are renormalized symmetrically with respect to the average values $\epsilon+\frac{U}{2}+\sigma \frac{B}{2}$ and $2 \Gamma$, respectively, for any complex frequency $E+i \omega$ with $\omega>0$.

Finally, we note that the stability constraint discussed in Sec. IID3 imposes a constraint on the RG flow: Since at any stage of the RG flow the effective Liouvillian $L(z)$ (191) can be calculated from the perturbative expansion (79), the imaginary parts of all nonzero eigenvalues of the renormalized $\bar{L}_{\Lambda}$ must be negative to avoid unphysical divergence of the time-dependent density operator. Such behavior would not go unnoticed in the RG since zero denominators would appear in the resolvents in Eq. (227), leading to an instability in the RG flow. This provides a simple criterion for the stability of the RG flow for the Anderson model that can be checked easily in numerical approximations. Although in previous applications of the RT-RG no instabilities have been reported, and in this study none were encountered either, the general conditions for stability are currently not known.

b. Fermionic supermatrix elements. The properties (216) and (217) strongly restrict the fermionic matrix elements of the resolvents $\Pi$ that can appear in the RG equations. We will see that implies that quite generally the RG equations decouple into smaller sets of equations (see Sec. IIIC) and that important simplifications arise in the $U=0$ limit (see Sec. IIIC3). These simplifications arise since in general on the right-hand side of RG equations such as (206) and (207), the resolvent $\Pi$ always appears sandwiched between pairs of $\bar{G}$ vertices (all are renormalized quantities but $\Lambda$ is not written). We list the different cases:

(i) In matrix elements of terms with only one resolvent $(X|\bar{G} \Pi \bar{G}| Y)$, there are no restrictions only if $X=Z_{R}, Y=$ $Z_{L}$. Indeed, upon inserting the completeness relation $1=$ $\left.\sum_{\eta, \sigma, v} \mid \alpha_{\eta \sigma}^{\nu}\right)\left(\alpha_{\eta \sigma}^{v} \mid+\right.$ (bosonic terms) left and right of $\Pi$, we see that according to Eqs. (216) and (217), all intermediate fermionic supermatrix elements contribute. However, when the basis supervectors $X, Y$ involve one of the supervectors $Z_{L}, Z_{R}$, then only certain matrix elements contribute: for 
$v= \pm$ these are

$$
\begin{array}{ll}
\left(\alpha_{\eta \sigma}^{v}\left|\Pi^{\alpha}\right| \alpha_{\eta \sigma}^{-}\right), & X=Z_{R}, \quad Y \neq Z_{L}, \\
\left(\alpha_{\eta \sigma}^{+}\left|\Pi^{\alpha}\right| \alpha_{\eta \sigma}^{v}\right), & X \neq Z_{R}, \quad Y=Z_{L},
\end{array}
$$

whereas, if the supervectors $Z_{L}, Z_{R}$ are not involved, only one factor is possible:

$$
\left(\alpha_{\eta \sigma}^{+}\left|\Pi^{\alpha}\right| \alpha_{\eta \sigma}^{-}\right), \quad X \neq Z_{R}, \quad Y \neq Z_{L} .
$$

(ii) In terms with $n \geqslant 3$ resolvents $(X|\bar{G} \Pi \bar{G} \ldots \bar{G} \Pi \bar{G}| Y)$, Eqs. (219) and (221) apply to the leftmost "boundary" resolvent. Otherwise, the expression vanishes for any $\Lambda$ since by Eq. (217) $\left(X|\bar{G} \Pi| \alpha^{+}\right)\left(\alpha^{+} \mid \bar{G} \Pi \bar{G} \cdots \propto\right.$ $\left(X|\bar{G} \Pi| \alpha^{+}\right)\left(Z_{L} \mid \Pi \bar{G} \cdots=0\right.$. Here, we used that $\left(Z_{L} \mid\right.$ is an exact eigenvector of $\bar{L}$, and thereby of $\Pi$ by Eq. (210), and a zero eigenvector of $\bar{G}$ by Eq. (212). Similarly, Eqs. (220) and (221) also apply to the rightmost "boundary" resolvents since $\left.\cdots \bar{G} \Pi \bar{G} \Pi \mid \alpha^{-}\right)\left(\alpha^{-}|\bar{G}| Y\right)=0$ by Eqs. (216), (211), and (213).

(iii) Finally, in terms with $n \geqslant 3$ resolvents, the resolvents that are not at the boundary can only contribute with fermionic matrix element $\left(\alpha_{\eta \sigma}^{+}\left|\Pi^{\alpha}\right| \alpha_{\eta \sigma}^{-}\right)$, irrespective of $X$ and $Y$ : this factor must always occur at least $n-2 \geqslant 1$ times.

\section{One-loop RG equations}

\section{Frequency dependence}

Since our goal is to calculate the stationary state from the effective Liouvillian $L(z)=\left.\bar{L}(E, \omega)\right|_{\Lambda=0}$ at $z=E+i \omega=i 0$, we first consider the RG equation for this quantity in the oneloop approximation and at frequency $E=0$ :

$$
\frac{d \bar{L}^{0}(0)}{d \Lambda}=i \frac{\Gamma}{\pi} \bar{G}_{1}^{0} \Pi^{0}\left(\bar{\mu}_{1}, \Lambda\right) \bar{G}_{\overline{1}}^{0} .
$$

Here, the superscript 0 indicates that we also evaluate the Liouvillian at zero reservoir frequency $\omega=0: \bar{L}^{0}(E):=$ $\bar{L}(E, 0)$. Similarly, in Eq. (222) we approximate the vertices by their initial values, as given by Eqs. (118) and (120), or by Eq. (51):

$$
\bar{G}_{1}(0,0, \Lambda) \approx \bar{G}_{\overline{1}}\left(\bar{\mu}_{1}, \Lambda,-\Lambda\right) \approx \bar{G}_{1}^{0}
$$

neglecting their dependence on the dot frequency $\left(E_{1}=\bar{\mu}_{1}\right)$, the reservoir frequency $(\omega=\Lambda)$, and the vertex-leg frequency $\left(\omega_{1}= \pm \Lambda\right)$. Such frequency dependencies arise only when accounting for the renormalization of the vertices: for small frequencies we can approximate in Eq. (207)

$$
\frac{d \bar{G}}{d \Lambda} \sim \frac{\Gamma}{\Lambda^{2}} \bar{G}^{3}
$$

on the right-hand side $\bar{G} \sim \bar{G}^{0} \sim 1$, giving $\bar{G}=\bar{G}^{0}+$ $O(\Gamma / \Lambda)$. In one-loop order for $\bar{L}$ one must therefore consistently neglect the renormalization of $\bar{G}$ [Eq. (223)], with respect to the log corrections to the Liouvillian that arise from Eq. (222). This will be checked later on. The resolvent is likewise evaluated at $\omega=\Lambda$. Note that the $\omega$ dependence of the resolvent in first approximation,

$$
\Pi^{0}(E, \omega)=\frac{1}{E+i \omega-\bar{L}^{0}(E)},
$$

does not originate from the Liouvillian.

In contrast, Eq. (222) depends on QD frequency $E$ in an important way: Due to the finite bias voltage, the renormalization of the zero $E$-frequency Liouvillian couples to the finitefrequency Liouvillian $E \rightarrow E_{1}=\bar{\mu}_{1}=\eta_{1} r_{1} V / 2$ appearing in Eq. (204) on the right-hand side. We therefore need to consider instead the following RG equations on a discrete grid of finite QD frequencies $E=k_{L} \bar{\mu}_{L}+k_{R} \bar{\mu}_{R}=\eta\left(k_{L}-k_{R}\right) V / 2$ :

$$
\frac{d \bar{L}^{0}(E)}{d \Lambda}=i \frac{\Gamma}{\pi} \bar{G}_{1}^{0} \Pi^{0}\left(E_{1}, \Lambda\right) \bar{G}_{\overline{1}}^{0},
$$

where $k_{L}, k_{R}=0,1,2, \ldots$ In the numerical calculations, we keep as many equations as required to make the solution converge with respect to the $k_{r}$. This coupling of the RG flow of the Liouvillian at energies differing by multiples of the voltage arises because the Matsubara frequencies of the different reservoirs are shifted by different electrochemical potentials: this is a typical feature of renormalization in a nonequilibrium system. ${ }^{26}$ We discuss the effect of neglecting the QD energy $E$ dependence in the RG equations in detail when we analyze the numerical results in Sec. IV B.

\section{Explicit one-loop RG equations for the Liouvillian}

Inserting the spectral decomposition for $\bar{L}(E)$ [cf. Eq. (161)] into Eq. (226),

$$
\frac{d \bar{L}^{0}(E)}{d \Lambda}=\frac{\Gamma}{\pi} \frac{1}{\Lambda-i \Theta_{1}^{k}} \bar{G}_{1}^{0} P_{1}^{k} \bar{G}_{\overline{1}}^{0},
$$

we obtain, abbreviating $\Theta_{1}^{k}=E_{1}-\lambda^{k}\left(E_{1}\right)$,

$$
\frac{d}{d \Lambda}\left[\kappa_{3}\left|\bar{L}^{0}(E)\right| \kappa_{0}\right]=\frac{\Gamma}{\pi}\left(\kappa_{3}|\mathcal{M}| \kappa_{0}\right),
$$

where $\left.\mid \kappa_{i}\right)$ are elements of the basis (91)-(96) and the supermatrix elements are sums $\left(\kappa_{1,2}\right.$ sums implicit) of factored contributions:

$$
\left(\kappa_{3}|\mathcal{M}| \kappa_{0}\right)=i \sum_{i}\left(\kappa_{3}\left|\bar{G}_{1}^{0}\right| \kappa_{2}\right)\left(\kappa_{1}\left|\bar{G}_{\overline{1}}^{0}\right| \kappa_{0}\right)\left(\kappa_{2}\left|\Pi_{1}^{i}\right| \kappa_{1}\right) .
$$

The product of $\bar{G}^{0}$ matrix elements gives a simple numerical factor 0 or \pm 1 [see expansions (118)-(120)], whereas the supermatrix elements

$$
i\left(\kappa_{2}\left|\Pi_{1}^{i}\right| \kappa_{1}\right)=\frac{1}{\Lambda-i \Theta_{1}^{i}}\left(\kappa_{2}\left|P_{1}^{i}\right| \kappa_{1}\right)
$$

arise from the spectral decomposition of the resolvent $\Pi^{0}\left(E_{1}, \Lambda\right)=\sum_{i} \Pi_{1}^{i}$. To explicitly sum over $\eta$ (contained in the multi-index $1=\eta, \sigma, r)$, which enters the resolvents only through $E_{\eta r}:=E_{1}=E-\eta \mu_{r}$, we abbreviate $\lambda^{i}\left(E_{1}\right):=\lambda_{\eta r}^{i}$, $P^{i}\left(E_{1}\right):=P_{\eta r}^{i}$, and

$$
i \Pi_{\eta r}^{i}:=\frac{P_{\eta r}^{i}}{\Lambda-i E_{\eta r}+i \lambda_{\eta r}^{i}} .
$$


Expanding Eq. (226) in the basis (91)-(96), we obtain

$$
\begin{aligned}
\frac{d \bar{L}^{0}(E)}{d \Lambda}= & \frac{i \Gamma}{\pi}\left[-\left(\left(\alpha_{-\bar{\sigma}}^{-}\left|\Pi_{-r}^{\alpha_{-\bar{\sigma}}}\right| \alpha_{-\bar{\sigma}}^{+}\right)+\left(\alpha_{+\sigma}^{-}\left|\Pi_{+r}^{\alpha_{+\sigma}}\right| \alpha_{+\sigma}^{+}\right)\right) \mid Z_{R}\right)\left(Z_{L}\left|+\left(\left(\alpha_{-\sigma}^{-}\left|\Pi_{-r}^{\alpha_{-\sigma}}\right| \alpha_{-\sigma}^{-}\right)-\left(\alpha_{+\bar{\sigma}}^{-}\left|\Pi_{+r}^{\alpha_{+\bar{\sigma}}}\right| \alpha_{+\bar{\sigma}}^{-}\right)\right)\right| Z_{R}\right)\left(\chi_{\sigma} \mid\right. \\
& \left.+\left(\left(\alpha_{-\bar{\sigma}}^{+}\left|\Pi_{-r}^{\alpha_{-\bar{\sigma}}}\right| \alpha_{-\bar{\sigma}}^{+}\right)-\left(\alpha_{+\sigma}^{+}\left|\Pi_{+r}^{\alpha_{+\sigma}}\right| \alpha_{+\sigma}^{+}\right)\right) \mid \chi_{\sigma}\right)\left(Z_{L}\left|-\left(\left(\alpha_{+\sigma}^{+}\left|\Pi_{+r}^{\alpha_{+\sigma}}\right| \alpha_{+\sigma}^{-}\right)+\left(\alpha_{-\bar{\sigma}}^{+}\left|\Pi_{-r}^{\alpha_{+\sigma}}\right| \alpha_{-\bar{\sigma}}^{-}\right)\right)\right| \chi_{\sigma}\right)\left(\chi_{\bar{\sigma}} \mid\right. \\
& \left.+\left(\left(\alpha_{+\sigma}^{+}\left|\Pi_{+r}^{\alpha_{+\sigma}}\right| \alpha_{+\sigma}^{-}\right)+\left(\alpha_{-\sigma}^{+}\left|\Pi_{-r}^{\alpha_{-\sigma}}\right| \alpha_{-\sigma}^{-}\right)\right) \mid S_{\sigma}\right)\left(S_{\sigma}\left|-\left(\alpha_{+\bar{\sigma}}^{+}\left|\Pi_{-r}^{\alpha_{+\bar{\sigma}}}\right| \alpha_{+\bar{\sigma}}^{-}\right)\right| T_{+}\right)\left(T_{+}\left|-\left(\alpha_{-\sigma}^{+}\left|\Pi_{+r}^{\alpha_{-\sigma}}\right| \alpha_{-\sigma}^{-}\right)\right| T_{-}\right)\left(T_{-} \mid\right. \\
& \left.-\left(\left(\chi_{\bar{\sigma}}\left|\Pi_{-r}^{\chi}\right| \chi_{\sigma}\right)+\left(T_{+}\left|\Pi_{+r}^{T_{+}}\right| T_{+}\right)-\left(S_{\sigma}\left|\Pi_{-r}^{S_{\sigma}}\right| S_{\sigma}\right)\right) \mid \alpha_{+\sigma}^{-}\right)\left(\alpha_{+\sigma}^{+} \mid\right. \\
& \left.-\left(\left(\chi_{\sigma}\left|\Pi_{+r}^{\chi}\right| \chi_{\bar{\sigma}}\right)+\left(T_{-}\left|\Pi_{-r}^{T_{-}}\right| T_{-}\right)-\left(S_{\sigma}\left|\Pi_{+r}^{S_{\sigma}}\right| S_{\sigma}\right)\right) \mid \alpha_{-\sigma}^{-}\right)\left(\alpha_{-\sigma}^{+} \mid\right]
\end{aligned}
$$

Here, we leave implicit the summation over $\sigma$ and $r$, as well as the summation over the two eigenvalues in the $\chi$ and $\alpha_{\eta \sigma}$ subspaces [cf. Eq. (161)]. The first two terms in the equation do not contribute to the calculation of the remaining terms of the effective Liouvillian or the transport current, but they are written here for completeness. Due to $\left(Z_{L} \mid \bar{G}=0\right.$ [cf. discussion of Eq. (85)], the zero eigenprojector $P^{Z_{L}}$ does not appear in Eq. (232), ensuring that none of the resolvents can diverge during the $\mathrm{RG}$ flow.

Using the relations $K\{\Lambda-i[z-\bar{L}(z)]\}^{-1} K=\{\Lambda-$ $\left.i\left[-z^{*}-\bar{L}\left(-z^{*}\right)\right]\right\}^{-1}$ [cf. Eqs. (70) and (117)], and by expanding resolvents into eigenprojectors using Eq. (231), we obtain the explicit RG equations for the Liouvillian expansion coefficients. We have a set of equations for 10 complex coefficients on an infinite, discrete grid of frequencies $E$ :

$$
\frac{d \xi_{\sigma, \bar{\sigma}}(E)}{d \Lambda}=i \frac{\Gamma}{\pi}\left(\frac{\left(\alpha_{-\bar{\sigma}}^{+}\left|P_{-r}^{\alpha_{-\bar{\sigma}}}\right| \alpha_{-\bar{\sigma}}^{-}\right)}{\Lambda-i\left(E_{-r}-\lambda_{-\bar{\sigma}}^{\alpha_{-\bar{\sigma}}}\right)}+\frac{\left(\alpha_{+\sigma}^{+}\left|P_{+r}^{\alpha_{+\sigma}}\right| \alpha_{+\sigma}^{-}\right)}{\Lambda-i\left(E_{+r}-\lambda_{+r}^{\alpha_{+\sigma}}\right)}\right)
$$

$$
\begin{gathered}
\frac{d E_{\sigma}(E)}{d \Lambda}=i \frac{\Gamma}{\pi}\left(\frac{\left(\alpha_{+\sigma}^{+}\left|P_{+r}^{\alpha_{+\sigma}}\right| \alpha_{+\sigma}^{-}\right)}{\Lambda-i\left(E_{+r}-\lambda_{+r}^{\alpha_{+\sigma}}\right)}+\frac{\left(\alpha_{-\sigma}^{+}\left|P_{-r}^{\alpha_{-\sigma}}\right| \alpha_{-\sigma}^{-}\right)}{\Lambda-i\left(E_{+r}-\lambda_{+r}^{\alpha_{+\sigma}}\right)}\right) \\
=\frac{d E_{\bar{\sigma}}^{*}\left(-E^{*}\right)}{d \Lambda}, \\
\frac{d M_{+}(E)}{d \Lambda}=-i \frac{\Gamma}{\pi} \frac{\left(\alpha_{+\bar{\sigma}}^{+}\left|P_{-r}^{\alpha_{+\bar{\sigma}}}\right| \alpha_{+\bar{\sigma}}^{-}\right)}{\Lambda-i\left(E_{-r}-\lambda_{-r}^{\alpha_{+\sigma}}\right)}, \\
\frac{d M_{-}(E)}{d \Lambda}=-i \frac{\Gamma}{\pi} \frac{\left(\alpha_{-, \sigma}^{+}\left|P_{+r}^{\alpha_{-\sigma}}\right| \alpha_{-, \sigma}^{-}\right)}{\Lambda-i\left(E_{+r}-\lambda_{+r}^{\alpha_{-\sigma}}\right)}=\frac{d M_{+}^{*}\left(-E^{*}\right)}{d \Lambda},
\end{gathered}
$$

$$
\begin{aligned}
\frac{d F_{+, \sigma}^{+-}(E)}{d \Lambda}= & -i \frac{\Gamma}{\pi}\left(\frac{\left(\chi_{\bar{\sigma}}\left|P_{-r}^{\chi}\right| \chi_{\sigma}\right)}{\Lambda-i\left(E_{-r}-\lambda_{-r}^{\chi}\right)}+\frac{\left(T_{+}\left|P_{+r}^{T_{+}}\right| T_{+}\right)}{\Lambda-i\left(E_{+r}-\lambda_{+}^{T_{+}}\right)}\right. \\
& \left.-\frac{\left(S_{\sigma}\left|P_{-r}^{S_{\sigma}}\right| S_{\sigma}\right)}{\Lambda-i\left(E_{-r}-\lambda_{-r}^{S_{\sigma}}\right)}\right), \\
\frac{d F_{-, \sigma}^{+-}(E)}{d \Lambda}= & -i \frac{\Gamma}{\pi}\left(\frac{\left(\chi_{\sigma}\left|P_{+}^{\chi}\right| \chi_{\bar{\sigma}}\right)}{\Lambda-i\left(E_{+r}-\lambda_{+}^{\chi}\right)}+\frac{\left(T_{-}\left|P_{-r}^{T_{-}}\right| T_{-}\right)}{\Lambda-i\left(E_{-r}-\lambda_{-r}^{T_{-}}\right)}\right. \\
& \left.-\frac{\left(S_{\sigma}\left|P_{+r}^{S_{\sigma}}\right| S_{\sigma}\right)}{\Lambda-i\left(E_{+r}-\lambda_{+}^{S_{\sigma}}\right)}\right)=-\frac{d\left[F_{+, \bar{\sigma}}^{+-}\left(-E^{*}\right)\right]^{*}}{d \Lambda} .
\end{aligned}
$$

Importantly, the eigenprojectors of the Liouvillian $\bar{L}^{0}$, $P_{\eta r}^{i}=P^{i}\left(E-\eta \mu_{r}\right)$ with eigenvalues $\lambda_{\eta r}^{i}=\lambda^{i}\left(E-\eta \mu_{r}\right)$ [cf. Eq. (148)] depend on the frequency $E-\eta \mu_{r}$. The explicit expressions for the projector matrix elements on the right-hand side are given in Eqs. (150), (152), (153), (155), and (159) and involve only the 10 coefficients appearing on the left-hand side. Equations (233)-(238) thus form a closed set of equations. This derives from the fact that the eigenprojectors of $\bar{L}_{\Lambda}$ that involve the zero eigensupervectors $Z_{L}$ and $Z_{R}$ drop out on the right-hand side by Eq. (161). Note that the fermionic matrix elements in the equations for the coefficients of bosonic terms that do not involve a $Z_{L}$ or $Z_{R}\left(\xi_{\sigma \sigma^{\prime}}, E_{\sigma}\right.$, and $\left.M_{\eta}\right)$ illustrate the simplification brought by Eq. (161).

The following five complex coefficients do not appear in the eigenvalues and projector matrix elements on the right-hand side of Eqs. (233)-(238). Their RG equations

$$
\begin{gathered}
\frac{d \psi_{\sigma}(E)}{d \Lambda}=i \frac{\Gamma}{\pi}\left(\frac{\left(\alpha_{-\bar{\sigma}}^{+}\left|P_{-r}^{\alpha_{-\bar{\sigma}}}\right| \alpha_{-\bar{\sigma}}^{+}\right)}{\Lambda-i\left(E_{-r}-\lambda_{-r}^{\alpha_{-\bar{\sigma}}}\right)}-\frac{\left(\alpha_{+\sigma}^{+}\left|P_{+r}^{\alpha_{+\sigma}}\right| \alpha_{+\sigma}^{+}\right)}{\Lambda-i\left(E_{+r}-\lambda_{+r}^{\alpha_{+\sigma}}\right)}\right), \\
\frac{d \zeta(E)}{d \Lambda}=-i \frac{\Gamma}{\pi}\left(\frac{\left(\alpha_{-\bar{\sigma}}^{-}\left|P_{-r}^{\alpha_{-\bar{\sigma}}}\right| \alpha_{-\bar{\sigma}}^{+}\right)}{\Lambda-i\left(E_{-r}-\lambda_{-r}^{\alpha_{-\bar{\sigma}}}\right)}+\frac{\left(\alpha_{+\sigma}^{-}\left|P_{+r}^{\alpha_{+\sigma}}\right| \alpha_{+\sigma}^{+}\right)}{\Lambda-i\left(E_{+r}-\lambda_{+r}^{\alpha_{+\sigma}}\right)}\right), \\
\frac{d \phi_{\sigma}(E)}{d \Lambda}=i \frac{\Gamma}{\pi}\left(\frac{\left(\alpha_{-\sigma}^{-}\left|P_{-r}^{\alpha_{-\sigma}}\right| \alpha_{-\sigma}^{-}\right)}{\Lambda-i\left(E_{-r}-\lambda_{-r}^{\alpha_{-\sigma}}\right)}-\frac{\left(\alpha_{+\bar{\sigma}}^{-}\left|P_{+r}^{\alpha_{+\bar{\sigma}}}\right| \alpha_{+\bar{\sigma}}^{-}\right)}{\Lambda-i\left(E_{+r}-\lambda_{+r}^{\alpha_{+\sigma}}\right)}\right)
\end{gathered}
$$

are therefore not required for the solution of Eqs. (233)-(238), but these coefficients $d o$ renormalize and depend on this solution. For the calculation of the current, only $\psi_{\sigma}$ is required. In contrast, the coefficients $\zeta$ and $\phi_{\sigma}$ are only required if one wishes to calculate, e.g., the stationary density matrix (151).

The remaining coefficients do not flow under the one-loop $\mathrm{RG}$, and remain at their initial values. For the two diagonal matrix elements of the coefficient matrix $\xi$ in the bosonic sector we have

$$
\frac{d \xi_{\sigma \sigma}}{d \Lambda}(E)=0,
$$

which is valid only within the present one-loop approximation. In contrast, for the remaining 16 fermionic coefficients, we have in general (e.g., also in two-loop order)

$$
\frac{d F_{\eta, \sigma}^{+ \pm}}{d \Lambda}(E)=\frac{d F_{\eta, \sigma}^{ \pm-}}{d \Lambda}(E)=0,
$$


due to the causal structure (cf. Secs. IID3 and III B4). We furthermore note that Eqs. (233)-(241) explicitly satisfy the Hermiticity conditions (144) and (147). Moreover, the proper transformation under charge and spin rotations is explicitly guaranteed by our use of irreducible tensor superoperators (cf. Sec. II C5b).

Finally, for the calculation of the current at a specific electrode $r= \pm$ (corresponding to $L, R$ ), we need the $\mathrm{RG}$ equations for the coefficients of the self-energy components $\bar{L}^{r}$ [cf. Eq. (209)]. These are simply obtained from the above equations by (i) giving all coefficients a superscript $r$ and (ii) suppressing the summation over $r$ contained in the multiindex 1 on the right-hand side, i.e., by setting $1=\eta, \sigma,+, \omega$.

Before we proceed to calculate the two-loop corrections to Eq. (222), we first show that already in the above one-loop approximation we obtain the exact solution for the current in the limit $U=0$. This is important since it demonstrates that for the current the two-loop corrections to $\bar{L}$, the one-loop corrections to the vertex $\bar{G}$, and the $\omega$ frequency dependence that we neglected here are intimately connected with interaction effects. We note, however, that for $U=0$ there are nonzero two-loop corrections which do not affect the current. $^{50}$

\section{Noninteracting case $U=0$ : Exact solution}

Without local interaction $U=0$, the Hamiltonian (11) is quadratic in the fermionic operators and the nonequilibrium Anderson model can be solved exactly in this limit. A solution using the real-time approach was reported in Refs. 63 and 64. We now show that (i) using the causal field superoperator algebra, one can obtain this solution within RT-RG framework and (ii) within the one-loop, frequency-independent approximation (222) this result is recovered upon careful inspection.

a. Exact current. In general, to calculate the current according to Eq. (189), we need the elements $\xi$ and $\psi_{\sigma}$ of the bosonic part of the effective Liouvillian $\bar{L}_{\Lambda}$. From these we can then easily find the other required coefficients $\xi^{r}$ and $\psi_{\sigma}^{r}$ of $\bar{L}_{\Lambda}^{r}$, which we do at the end. The coefficients $\xi$ and $\psi_{\sigma}$ in turn require the solution of Eqs. (233)-(238), which we first discuss. Then, we show that higher-order correction as well as frequency corrections that we neglected in deriving Eqs. (233)-(238) have no influence on the stationary current. We start by noting that on the right-hand side of the $R G$ equations (233)-(236) for the bosonic sector, only fermionic intermediate states appear in the resolvent matrix elements. We therefore first calculate the eigenvalues of the fermionic projectors to which these coefficients couple. From Eq. (163) it follows that, for $U=0$,

$$
\begin{gathered}
\lambda^{\alpha_{\eta \sigma}, \pm}=\eta \epsilon+\sigma \frac{B}{2}-2 i \Gamma \pm i \Gamma, \\
P^{\alpha_{\eta \sigma}, \pm}=\frac{1}{2}\left(\alpha_{\eta \sigma}^{0} \pm \eta \alpha_{\eta \sigma}^{3} \mp \eta \frac{\Delta F_{\eta, \sigma}^{-+}}{\Gamma} \alpha_{\eta \sigma}^{-}\right) .
\end{gathered}
$$

There are three important points. (i) Since $U=0$ the eigenvalues are independent of $\Delta F_{\eta, \sigma}^{-+}$, i.e., they are not renormalized (cf. Sec. IID3) and therefore do not acquire a frequency dependence. (ii) The right-hand side of the RG equation for any bosonic superoperator that is relevant to the current, i.e., excluding $\zeta, \phi$, but with the exception of $\psi_{\sigma}$ contains the off-diagonal super matrix elements

$$
\left(\alpha_{\eta \sigma}^{+}\left|\Pi_{1}^{\alpha_{\eta \sigma}}\right| \alpha_{\eta \sigma}^{-}\right)=0
$$

as a factor by the general property (220). This matrix element vanishes for $U=0$ by Eq. (245), implying that these coefficients do not renormalize in any higher loop order since such coefficients always contain this factor on the right-hand side of Eq. (206) at least once by Eq. (221). (iii) In contrast, the renormalization of the quantities $\psi_{\sigma}$ at $E=0$ involve fermionic virtual states with the simple factors

$$
\begin{aligned}
& i\left(\alpha_{+\sigma}^{+}\left|\Pi_{+r}^{\alpha_{+\sigma}}\right| \alpha_{+\sigma}^{+}\right) \\
& \quad=\sum_{r}\left(\alpha_{+\sigma}^{+}\left|\frac{1}{\Lambda-i r V / 2+i \lambda_{+r}^{\alpha_{+\sigma}}(r V / 2)}\right| \alpha_{+\sigma}^{+}\right) \\
& \quad=\sum_{r} \frac{1}{\Lambda+\Gamma+i\left(\epsilon_{\sigma}-r V / 2\right)},
\end{aligned}
$$

where we defined $\epsilon_{\sigma}:=\epsilon+\sigma B / 2$. Importantly, for $U=$ 0 these matrix elements do not vanish, but they become independent of $\Delta F_{\eta, \sigma}^{-+}$. Higher-loop corrections for $\psi_{\sigma}$ vanish since they contain the factor (246) at least once by Eq. (219). Therefore, Eq. (239) is the exact RG equation for $\psi_{\sigma}$ at $E=0$ and for $U=0$ :

$$
\begin{aligned}
\frac{d \psi_{\sigma}(0)}{d \Lambda} & =\sum_{r} \frac{2 \Gamma}{\pi} \operatorname{Im}\left(\alpha_{+\sigma}^{+}\left|\mathrm{i} \Pi_{+\mathrm{r}}^{\alpha_{+\sigma}}\right| \alpha_{+\sigma}^{+}\right) \\
& =\sum_{r} \frac{2 \Gamma}{\pi} \frac{\epsilon_{\sigma}-r V / 2}{(\Lambda+\Gamma)^{2}+\left(\epsilon_{\sigma}-r V / 2\right)^{2}},
\end{aligned}
$$

where we again used the $K$-conjugation properties (70) and (117). With the initial value $\psi_{\sigma, \Lambda=\infty}=0$ [cf. Eq. (134)], we obtain

$$
\psi_{\sigma}(0)=-\sum_{r} \frac{2 \Gamma}{\pi} \arctan \left(\frac{\epsilon_{\sigma}-r V / 2}{\Gamma}\right) .
$$

Leaving out the summation over the electrode $r$ in the above calculation, we obtain the coefficients of the self-energy component $\left.\bar{L}_{\Lambda}^{r}\right|_{\Lambda=0}=\Sigma^{r}(0)$, required for the current [cf. Eq. (189)],

$$
\left\langle I^{r}\right\rangle=\frac{1}{\sqrt{2}}\left[\left(T_{0} \mid \vec{\psi}^{r}\right)-\left(T_{0}\left|\xi^{r} \xi^{-1}\right| \vec{\psi}\right)\right]=\frac{1}{4} \sum_{\sigma}\left(\psi_{\sigma}^{r}-\psi_{\sigma}^{\bar{r}}\right),
$$

using $\frac{1}{\sqrt{2}}\left(T_{0} \mid=\frac{1}{2} \sum_{\sigma}\left(\chi_{\sigma} \mid\right.\right.$ and $\xi^{r}=\xi / 2$, giving the current

$$
\langle I\rangle=\sum_{r, \sigma= \pm} r \frac{\Gamma}{2 \pi} \arctan \left(\frac{\epsilon_{\sigma}+r V / 2}{\Gamma}\right)
$$

and the nonlinear differential conductance

$$
\frac{d I}{d V}=\frac{1}{4 \pi} \sum_{r, \sigma= \pm} \frac{\Gamma^{2}}{\Gamma^{2}+\left(\epsilon_{\sigma}+r V / 2\right)^{2}}
$$

in our units $e=1, \hbar=1$. Restoring Gaussian units, the current and conductance prefactors become $\frac{\Gamma}{2 \pi} \rightarrow \Gamma \frac{e}{\hbar}, \frac{\Gamma}{2 \pi} \rightarrow \Gamma \frac{e^{2}}{2 \hbar}$ giving in linear response a conductance of $e^{2} / h$ per spin channel. The importance of recovering this exact result for $U=0$ is that already at this level of approximation, our RT-RG approach captures correctly the weak-interaction limit $U \ll \Gamma$ while treating the tunneling nonperturbatively in $\Gamma$. Moreover, 
it shows that the two-loop corrections to the Liouvillian and one-loop corrections to the vertices that affect the stationary current are generated by the Coulomb interaction. In general, however, the noninteracting limit requires a two-loop treatment.

\section{Two-loop RG equations}

\section{Vertex frequency dependence}

We concluded in Sec. III C1 that in the two-loop approximation for the Liouvillian one should consider the vertex renormalization and $\omega$ dependence of both $\bar{G}$ and $\bar{L}$. Indeed, we find below that these effects are comparable and involve important cancellations. By systematically expanding about the frequency-independent bare vertex $\bar{G}^{0}$, we can incorporate the vertex corrections into a single effective equation for the two-loop Liouvillian (272) below. We proceed in three steps:

Step 1. The starting point is the one-loop approximation defined by Eq. (226) for any $E$. This we use to calculate a first approximation for the $\omega$ dependence of both the Liouvillian and the propagator. We expand

$$
\begin{gathered}
\bar{L}(E, \omega) \approx \bar{L}^{0}(E)+\bar{L}^{1}(E, \omega), \\
\Pi(E, \omega) \approx \Pi^{0}(E, \omega)+\Pi^{1}(E, \omega) .
\end{gathered}
$$

The one-loop equation accounting for the leading frequency dependence is obtained by setting $G \approx G^{0}$ and $\Pi(E, \omega) \approx$ $\Pi^{0}(E, \omega)$ in the one-loop part of Eq. (206):

$$
\frac{d \bar{L}(E, \omega)}{d \Lambda}=i \frac{\Gamma}{\pi} \bar{G}_{1}^{0} \Pi^{0}\left(E_{1}, \Lambda+\omega\right) \bar{G}_{\overline{1}}^{0} .
$$

Subtracting Eq. (226), we obtain

$$
\frac{d \bar{L}^{1}(E, \omega)}{d \Lambda} \approx i \frac{\Gamma}{\pi} \bar{G}_{1}^{0}\left[\Pi^{0}\left(E_{1}, \omega+\Lambda\right)-\Pi^{0}\left(E_{1}, \Lambda\right)\right] \bar{G}_{\overline{1}}^{0} .
$$

Shifting the integration variable in the $\Pi\left(E_{1}, \Lambda\right)$ term, we obtain in the wide-band limit (which we assume throughout)

$$
\bar{L}^{1}(E, \omega) \approx \int_{\Lambda-\omega}^{\Lambda} i \frac{\Gamma}{\pi} \bar{G}_{1}^{0} \Pi^{0}\left(E_{1}, \omega+\Lambda\right) \bar{G}_{\overline{1}}^{0} .
$$

Equation (257) does not need to be evaluated further since it cancels out below. Note that the correction vanishes at zero frequency, $\bar{L}^{1}(E, 0)=0$, for all $E$ as required. Expanding the full resolvents (204) with the approximation (254) to the first order in $\bar{L}^{1}(E, \omega)$, we obtain

$$
\Pi^{1}(E, \omega)=\Pi^{0}(E, \omega) \bar{L}^{1}(E, \omega) \Pi^{0}(E, \omega) .
$$

Step 2. In a similar way, we now calculate the $\omega$ corrections in the leading one-loop order for the vertices:

$$
\bar{G}_{1}\left(E, \omega, \omega_{1}\right) \approx \bar{G}^{0}+\bar{G}_{1}^{1}\left(E, \omega, \omega_{1}\right) .
$$

Keeping only the leading term on the right-hand side of Eq. (207), we obtain with the same approximations as above

$$
\begin{aligned}
\bar{G}_{1}^{1}\left(E, \omega, \omega_{1}\right)= & -i \frac{\Gamma}{\pi} \int_{D}^{\Lambda} d \Lambda^{\prime} \bar{G}_{2}^{0} \Pi^{0}\left(E_{2}, \omega+\Lambda^{\prime}\right) \\
& \times \bar{G}_{1}^{0} \Pi^{0}\left(E_{12}, \omega+\omega_{1}+\Lambda^{\prime}\right) \bar{G}_{\overline{2}}^{0} .
\end{aligned}
$$

We stress that the argument under the integral depends on $\Lambda^{\prime}$ both through the explicit arguments as well as through the cutoff dependence of $\bar{L}_{\Lambda^{\prime}}\left(G^{0}\right.$ is the bare vertex). Restoring the latter explicitly,

$$
\Pi_{\Lambda^{\prime}}^{0}\left(E, \omega+\Lambda^{\prime}\right)=\frac{1}{\omega+\Lambda^{\prime}-i \bar{L}_{\Lambda^{\prime}}^{0}(E)} .
$$

Since this is only important at this point, we stick with the implicit notation.

Step 3. Using the expansions for the resolvents (258) and vertices (260), we can now calculate an approximation to the right-hand side of Eq. (206), keeping the leading-order frequency corrections:

$$
\frac{d \bar{L}}{d \Lambda}=\frac{d \bar{L}^{\text {(one loop) }}}{d \Lambda}+\frac{d \bar{L}^{\text {(two loop) }}}{d \Lambda}+\frac{d \bar{L}^{\text {(vertex) }}}{d \Lambda},
$$

where all terms are written at frequencies $E$ and $\omega$ and

$$
\frac{d \bar{L}^{\text {(one loop) }}}{d \Lambda}=i \frac{\Gamma}{\pi}\left[\bar{G}_{1}^{0} \Pi^{0}\left(E_{1}, \Lambda\right) \bar{G}_{\overline{1}}^{0}+\bar{G}_{1}^{0} \Pi^{1}\left(E_{1}, \Lambda\right) \bar{G}_{\overline{1}}^{0}\right]
$$

are the terms appearing from the expansion of the one-loop diagram in: ${ }^{1}$

$$
\begin{aligned}
\frac{d \bar{L}^{\text {(two loop) }}}{d \Lambda}= & \frac{\Gamma^{2}}{\pi^{2}} \bar{G}_{1}^{0} \Pi^{0}\left(E_{1}, \Lambda\right) \bar{G}_{2}^{0} \\
& \times \Pi^{0}\left(E_{12}, \Lambda+\omega_{2}\right) \bar{G}_{\overline{2}}^{0} \Pi^{0}\left(E_{1}, \Lambda\right) \bar{G}_{\overline{1}}^{0}
\end{aligned}
$$

is a two-loop term with bare vertices, and

$$
\begin{aligned}
\frac{d \bar{L}^{\text {(vertex })}}{d \Lambda}= & i \frac{\Gamma}{\pi} \bar{G}_{1}^{1}(E, 0, \Lambda) \Pi^{0}\left(E_{1}, \Lambda\right) \bar{G}_{\overline{1}}^{0} \\
& +i \frac{\Gamma}{\pi} \bar{G}_{1}^{0} \Pi^{0}\left(E_{1}, \Lambda\right) \bar{G}_{\overline{1}}^{1}\left(E_{1}, \Lambda,-\Lambda\right)
\end{aligned}
$$

are the terms appearing from the expansion of $\bar{G}$ in $\bar{G}^{1}$ in the one-loop diagram. Inserting Eq. (257) into the one-loop Liouvillian frequency correction [last term in Eq. (263)], we see that it exactly cancels the two-loop term (264). Neither term therefore needs to be calculated, simplifying the approach to a great extent.

The above holds for any $E$ and $\omega$ : integrating Eq. (262) at $\omega=0$ using the above calculated right-hand side we obtain a new approximation for $\bar{L}^{0}(E)$, improving over our initial approximation based on the one-loop equation (226). In principle, steps 1 and 2 should be repeated, resulting in corrections of higher orders, which we neglect. We thus equate $d \bar{L} / d \Lambda \approx \bar{L}^{0} / d \Lambda$ on the left-hand side of Eq. (262). We obtain a central result of this section: a single effective two-loop RG equation for the Liouvillian at $\omega=0$ :

$$
\begin{aligned}
\frac{d \bar{L}^{0}(E)}{d \Lambda}= & i \frac{\Gamma}{\pi} \bar{G}_{1}^{0} \Pi^{0}\left(E_{1}, \Lambda\right) \bar{G}_{\overline{1}}^{0} \\
& +i \frac{\Gamma}{\pi} \bar{G}_{1}^{1}(E, 0, \Lambda) \Pi^{0}\left(E_{1}, \Lambda\right) \bar{G}_{\overline{1}}^{0} \\
& +i \frac{\Gamma}{\pi} \bar{G}_{1}^{0} \Pi^{0}\left(E_{1}, \Lambda\right) \bar{G}_{\overline{1}}^{1}\left(E_{1}, \Lambda,-\Lambda\right) .
\end{aligned}
$$

Notably, due to the cancellation the entire leading reservoir $(\omega)$ frequency dependence comes from the vertex corrections (260). This single equation yields a significant simplification over the coupled integrodifferential equations (206) and (207). The equation (267) for $\bar{L}$ can be converted into 
a differential RG equation by analytically performing the integral in Eq. (260) (see Sec. III D2). Furthermore, since we can work with the bare vertex superoperators, we can make use of their simple anticommutation relations (53), which are not preserved under the RG (in contrast to other useful properties of the vertex, see Appendix G). The QD frequency $(E)$ dependence in Eq. (267) is of the same type as for the one-loop equations (232): the RG equation for $L^{0}(0)$ depends on $L^{0}\left(\bar{\mu}_{1}\right)=L^{0}\left(\eta_{1} r_{1} V / 2\right)$, etc. It therefore has to be solved in the same way by including multiple Matsubara axes and converging the energy hierarchy of equations (see Secs. III C1 and IV). Finally, we also note that one can indeed neglect the frequency dependence generated by vertex renormalization since it is indeed small, as we assumed in our derivation of the one-loop equations. This can be seen if one substitutes the calculated correction (260) into (207). Here, we anticipate the projector expansion of $\bar{G}^{1}$ [Eq. (271)]: it is seen that $\bar{G}^{1}$ is a well-behaved function of the cutoff and frequency, decaying at small $\Lambda$. It generates only small corrections in agreement with our approximation (224).

\section{Explicit two-loop RG equations for the Liouvillian}

To obtain a differential RG equation from Eq. (267), the integration in Eq. (260) needs to be performed. This is complicated by the implicit $\Lambda^{\prime}$ dependence of the propagators that we pointed out with Eq. (261). We now make an adiabatic approximation by expanding only this dependence about
$\Lambda^{\prime}=\Lambda$, i.e., we substitute

$$
\bar{L}_{\Lambda^{\prime}} \approx \bar{L}_{\Lambda}
$$

in Eq. (261) and neglect corrections $\sim d \bar{L}_{\Lambda^{\prime}} / d \Lambda$, which, by the RG equation (267), are of higher order and should therefore be neglected. To preserve the compact form of the equations, we define

$$
\begin{gathered}
\Theta_{1 \ldots n}^{i}=E_{1 \ldots n}-\lambda^{i}\left(E_{1 \ldots n}\right), \\
P_{1 \ldots n}^{i}=P^{i}\left(E_{1 \ldots n}\right),
\end{gathered}
$$

where $P^{i}(E)$ and $\lambda^{i}(E)$ are eigenprojectors and eigenvalues of Eq. (148) at cutoff $\Lambda$ (not $\Lambda^{\prime}$ ) and we will implicitly sum over all appearing eigenvalue labels $i, j, k$ below. To perform the integral, we insert the projector expansion (148) of $\bar{L}$ evaluated at $\Lambda$ under the integral and obtain the explicit $\omega$-dependent vertex correction

$$
\begin{aligned}
& \bar{G}_{1}^{1}\left(E, \omega, \omega_{1}\right) \\
& =-i \frac{\Gamma}{\pi} \int_{D}^{\Lambda} d \Lambda^{\prime} \frac{\bar{G}_{2}^{0} P_{2}^{i} \bar{G}_{1}^{0} P_{12}^{j} \bar{G}_{\overline{2}}^{0}}{\left(\Lambda^{\prime}+\omega-i \Theta_{2}^{i}\right)\left(\Lambda^{\prime}+\omega+\omega_{1}-i \Theta_{12}^{j}\right)} \\
& =i \frac{\Gamma}{\pi} \frac{\bar{G}_{2}^{0} P_{2}^{i} \bar{G}_{1}^{0} P_{12}^{j} \bar{G}_{2}^{0}}{\omega_{1}-i\left(\Theta_{12}^{j}-\Theta_{2}^{i}\right)} \ln \left(\frac{\Lambda+\omega-i \Theta_{2}^{i}}{\Lambda+\omega+\omega_{1}-i \Theta_{12}^{j}}\right) .
\end{aligned}
$$

Combining the rest of Eq. (263) with Eq. (265), we obtain

$$
\begin{aligned}
\frac{d \bar{L}^{0}(E)}{d \Lambda}= & \frac{\Gamma}{\pi} \frac{1}{\Lambda-i \Theta_{1}^{k}} \bar{G}_{1}^{0} P_{1}^{k} \bar{G}_{\overline{1}}^{0}-i \frac{\Gamma^{2}}{\pi^{2}} \frac{1}{\Lambda-i \Theta_{1}^{k}} \frac{1}{\Lambda-i\left(\Theta_{12}^{j}-\Theta_{2}^{i}\right)} \\
& \times \ln \left(\frac{2 \Lambda-i \Theta_{12}^{j}}{\Lambda-i \Theta_{2}^{i}}\right)\left[\bar{G}_{1}^{0} P_{1}^{k} \bar{G}_{2}^{0} P_{12}^{j} \bar{G}_{\overline{1}}^{0} P_{2}^{i} \bar{G}_{\overline{2}}^{0}+\bar{G}_{2}^{0} P_{2}^{i} \bar{G}_{1}^{0} P_{12}^{j} \bar{G}_{\overline{2}}^{0} P_{1}^{k} \bar{G}_{\overline{1}}^{0}\right]
\end{aligned}
$$

This is a central result of the paper. The explicit evaluation of Eq. (272) for the Anderson model is required for our numerical implementation, but also allows us to draw some general conclusions about the two-loop (and higher) corrections. The one-loop part is given by Eq. (226) and we proceed analogously for the two-loop part:

$$
\left.\left.\frac{d \bar{L}^{0}(E)}{d \Lambda}\right|_{\text {two loop }}=-i \frac{\Gamma^{2}}{\pi^{2}}\left(\kappa_{7}|\mathcal{M}| \kappa_{0}\right) \mid \kappa_{7}\right)\left(\kappa_{0} \mid\right.
$$

where the supermatrix elements are factored as follows:

$$
\left(\kappa_{7}|\mathcal{M}| \kappa_{0}\right)=\sum_{\kappa_{6} \ldots \kappa_{1}}\left(\kappa_{7}\left|\bar{G}_{1}^{0}\right| \kappa_{6}\right)\left(\kappa_{5}\left|\bar{G}_{2}^{0}\right| \kappa_{4}\right)\left(\kappa_{3}\left|\bar{G}_{\overline{1}}^{0}\right| \kappa_{2}\right)\left(\kappa_{1}\left|\bar{G}_{\overline{2}}^{0}\right| \kappa_{0}\right) \mathcal{N}\left(\left(\kappa_{6}\left|P_{1}^{k}\right| \kappa_{5}\right),\left(\kappa_{4}\left|P_{12}^{j}\right| \kappa_{3}\right),\left(\kappa_{2}\left|P_{2}^{i}\right| \kappa_{1}\right)\right) .
$$

The product of $\bar{G}^{0}$ matrix elements gives a simple numerical factor, whereas the remaining part

$$
\mathcal{N}\left(\left(\kappa_{6}\left|P_{1}^{k}\right| \kappa_{5}\right),\left(\kappa_{4}\left|P_{12}^{j}\right| \kappa_{3}\right),\left(\kappa_{2}\left|P_{2}^{i}\right| \kappa_{1}\right)\right)=\mathcal{S}\left(\Theta_{1}^{k}, \Theta_{2}^{i}, \Theta_{12}^{j}\right)\left(\kappa_{6}\left|P_{1}^{k}\right| \kappa_{5}\right)\left(\kappa_{4}\left|P_{12}^{j}\right| \kappa_{3}\right)\left(\kappa_{2}\left|P_{2}^{i}\right| \kappa_{1}\right)
$$

contains the product of the nontrivial projector matrix elements and the propagator factors

$$
\mathcal{S}\left(\Theta_{1}^{k}, \Theta_{2}^{i}, \Theta_{12}^{j}\right)=\frac{\ln \left(\frac{2 \Lambda-i \Theta_{12}^{j}}{\Lambda-i \Theta_{2}^{i}}\right)}{\left(\Lambda-i \Theta_{1}^{k}\right)\left[\Lambda-i\left(\Theta_{12}^{j}-\Theta_{2}^{i}\right)\right]}+\left(\Theta_{1}^{k} \leftrightarrow \Theta_{2}^{i}\right) .
$$

The argument of this function is constructed by formally putting the variables containing the eigenvalues of the three projectors in the argument of $\mathcal{N}$ into the corresponding arguments of the scalar function $\Theta$. With this we can give explicit expressions for Eq. (273). It is shown in Appendix G how the conservation of Hermiticity by the self-energy can be used to minimize the number 
of terms to be calculated. For the right-hand side of Eq. (273), we now explicitly list half of the terms:

$$
\begin{aligned}
& \left.-\mid \chi_{\sigma}\right)\left(\chi_{\sigma} \mid\left[\mathcal{N}\left(\left(\alpha_{+\sigma}^{+}\left|P_{1}^{\alpha_{+\sigma}}\right| \alpha_{+\sigma}^{-}\right),\left(T_{+}\left|P_{12}^{T}\right| T_{+}\right),\left(\alpha_{+\bar{\sigma}}^{+}\left|P_{2}^{\alpha_{+\bar{\sigma}}}\right| \alpha_{+\bar{\sigma}}^{-}\right)\right)+\mathcal{N}\left(\left(\alpha_{+\sigma}^{+}\left|P_{1}^{\alpha_{+\sigma}}\right| \alpha_{+\sigma}^{-}\right),\left(S_{\sigma}\left|P_{1 \overline{2}}^{S}\right| S_{\sigma}\right),\left(\alpha_{-\sigma}^{+}\left|P_{\overline{2}}^{\alpha_{-\sigma}}\right| \alpha_{-\sigma}^{-}\right)\right)\right]\right. \\
& \left.+\mid \chi_{\sigma}\right)\left(\chi_{\bar{\sigma}} \mid \mathcal{N}\left(\left(\alpha_{+\sigma}^{+}\left|P_{1}^{\alpha_{+\sigma}}\right| \alpha_{+\sigma}^{-}\right),\left(\chi_{\bar{\sigma}}\left|P_{1 \overline{1}}^{\chi}\right| \chi_{\sigma}\right),\left(\alpha_{-\bar{\sigma}}^{+}\left|P_{\overline{1}}^{\alpha_{-\bar{\sigma}}}\right| \alpha_{-\bar{\sigma}}^{-}\right)\right)\right. \\
& \left.-\mid \chi_{\sigma}\right)\left(Z_{L} \mid\left[\mathcal{N}\left(\left(\alpha_{+\sigma}^{+}\left|P_{1}^{\alpha_{+\sigma}}\right| \alpha_{+\sigma}^{-}\right),\left(T_{+}\left|P_{12}^{T}\right| T_{+}\right),\left(\alpha_{+\bar{\sigma}}^{+}\left|P_{2}^{\alpha_{+\bar{\sigma}}}\right| \alpha_{+\bar{\sigma}}^{+}\right)\right)-\mathcal{N}\left(\left(\alpha_{+\sigma}^{+}\left|P_{1}^{\alpha_{+\sigma}}\right| \alpha_{+\sigma}^{-}\right),\left(S_{\sigma}\left|P_{1 \overline{2}}^{S}\right| S_{\sigma}\right),\left(\alpha_{-\sigma}^{+}\left|P_{\overline{2}}^{\alpha_{-\sigma}}\right| \alpha_{-\sigma}^{+}\right)\right)\right.\right. \\
& \left.+\mathcal{N}\left(\left(\alpha_{+\sigma}^{+}\left|P_{1}^{\alpha_{+\sigma}}\right| \alpha_{+\sigma}^{-}\right),\left(\chi_{\bar{\sigma}}\left|P_{1 \overline{1}}^{\chi}\right| \chi_{\sigma}\right),\left(\alpha_{-\bar{\sigma}}^{+}\left|P_{\overline{1}}^{\alpha_{-\bar{\sigma}}}\right| \alpha_{-\bar{\sigma}}^{+}\right)\right)\right] \\
& \left.-\mid S_{\sigma}\right)\left(S_{\sigma} \mid\left[\mathcal{N}\left(\left(\alpha_{+\sigma}^{+}\left|P_{1}^{\alpha_{+\sigma}}\right| \alpha_{+\sigma}^{-}\right),\left(T_{+}\left|P_{12}^{T}\right| T_{+}\right),\left(\alpha_{+\sigma}^{+}\left|P_{2}^{\alpha_{+\sigma}}\right| \alpha_{+\sigma}^{-}\right)\right)+\mathcal{N}\left(\left(\alpha_{+\sigma}^{+}\left|P_{1}^{\alpha_{+\sigma}}\right| \alpha_{+\sigma}^{-}\right),\left(\chi_{\bar{\sigma}}\left|P_{1 \overline{2}}^{\chi}\right| \chi_{\bar{\sigma}}\right),\left(\alpha_{-\sigma}^{+}\left|P_{\overline{2}}^{\alpha_{-\sigma}}\right| \alpha_{-\sigma}^{-}\right)\right)\right]\right. \\
& \left.-\mid T_{-}\right)\left(T_{-} \mid\left[\mathcal{N}\left(\left(\alpha_{-\sigma}^{+}\left|P_{1}^{\alpha_{-\sigma}}\right| \alpha_{-\sigma}^{-}\right),\left(S_{\sigma}\left|P_{12}^{S}\right| S_{\sigma}\right),\left(\alpha_{-\sigma}^{+}\left|P_{2}^{\alpha_{-\sigma}}\right| \alpha_{-\sigma}^{-}\right)\right)+\mathcal{N}\left(\left(\alpha_{-\bar{\sigma}}\left|P_{1}^{\alpha_{-\bar{\sigma}}}\right| \alpha_{-\bar{\sigma}}^{-}\right),\left(\chi_{\bar{\sigma}}\left|P_{12}^{\chi}\right| \chi_{\bar{\sigma}}\right),\left(\alpha_{-\sigma}^{+}\left|P_{2}^{\alpha_{-\sigma}}\right| \alpha_{-\sigma}^{-}\right)\right)\right]\right. \\
& \left.+\mid \alpha_{+\sigma}^{-}\right)\left(\alpha_{+\sigma}^{+} \mid\left[\mathcal{N}\left(\left(T_{+}\left|P_{1}^{T}\right| T_{+}\right)\left(\alpha_{+\sigma}^{+}\left|P_{1 \overline{1}}^{\alpha_{+\sigma}}\right| \alpha_{+\sigma}^{-}\right),\left(S_{\sigma}\left|P_{\overline{1}}^{S}\right| S_{\sigma}\right)\right)+\mathcal{N}\left(\left(T_{+}\left|P_{1}^{T}\right| T_{+}\right),\left(\alpha_{+\bar{\sigma}}^{+}\left|P_{1 \overline{2}}^{\alpha_{+\bar{\sigma}}}\right| \alpha_{+\bar{\sigma}}^{-}\right),\left(\chi_{\sigma}\left|P_{\overline{2}}^{\chi}\right| \chi_{\sigma}\right)\right)\right]\right. \\
& \left.+\mid \alpha_{-\sigma}^{-}\right)\left(\alpha_{-\sigma}^{+} \mid\left[\mathcal{N}\left(\left(\chi_{\sigma}\left|P_{1}^{\chi}\right| \chi_{\sigma}\right),\left(\alpha_{+\sigma}^{+}\left|P_{12}^{\alpha_{+\sigma}}\right| \alpha_{+\sigma}^{-}\right),\left(S_{\sigma}\left|P_{2}^{S}\right| S_{\sigma}\right)\right)+\mathcal{N}\left(\left(\chi_{\sigma}\left|P_{1}^{\chi}\right| \chi_{\sigma}\right),\left(\alpha_{-\bar{\sigma}}^{+}\left|P_{1 \overline{2}}^{\alpha_{\bar{\sigma}}}\right| \alpha_{-\bar{\sigma}}^{-}\right),\left(T_{-}\left|P_{\overline{2}}^{T}\right| T_{-}\right)\right)\right.\right. \\
& +\mathcal{N}\left(\left(S_{\sigma}\left|P_{1}^{S}\right| S_{\sigma}\right),\left(\alpha_{-\sigma}^{+}\left|P_{1 \overline{1}}^{\alpha_{-\sigma}}\right| \alpha_{-\sigma}^{-}\right),\left(T_{-}\left|P_{\overline{1}}^{T}\right| T_{-}\right)\right)+\mathcal{N}\left(\left(S_{\sigma}\left|P_{1}^{S}\right| S_{\sigma}\right),\left(\alpha_{+\sigma}^{+}\left|P_{12}^{\alpha_{+\sigma}}\right| \alpha_{+\sigma}^{-}\right),\left(\chi_{\bar{\sigma}}\left|P_{2}^{\chi}\right| \chi_{\bar{\sigma}}\right)\right) \\
& \left.-\mathcal{N}\left(\left(\chi_{\sigma}\left|P_{1}^{\chi}\right| \chi_{\bar{\sigma}}\right),\left(\alpha_{+\bar{\sigma}}^{+}\left|P_{12}^{\alpha_{+\bar{\sigma}}}\right| \alpha_{+\bar{\sigma}}^{-}\right),\left(\chi_{\sigma}\left|P_{2}^{\chi}\right| \chi_{\bar{\sigma}}\right)\right)\right] \text {. }
\end{aligned}
$$

Here, we use the notation

$$
P_{i}^{k}=P^{k}\left(-\eta_{i} \mu_{i}\right), \quad P_{i j}^{k}=P^{k}\left(-\eta_{i} \mu_{i}-\eta_{j} \mu_{j}\right)
$$

We fixed the particle-hole index $\eta$ in the multi-indices as

$$
\eta_{i}= \begin{cases}+, & i=1,2 \\ -, & i=\overline{1}, \overline{2}\end{cases}
$$

All other indices in Eqs. (277)-(283) are implicitly summed over. The other half of the terms of Eq. (273) can be constructed in the same way by taking into account the opposite sign of $\eta_{1}$ using the recipe of Appendix G. For the calculation of the current, only the leftmost reservoir index $r_{1}$ should not be summed over.

We presented Eqs. (277)-(283) in order to show a number of important general properties. First, in the fermionic sector (281)-(283), the structure is the same as for the one-loop equation, i.e., only terms $\left.\mid \alpha_{\eta \sigma}^{-}\right)\left(\alpha_{\eta \sigma}^{+} \mid\right.$appear as discussed (Sec. III B3). This property holds in any order of RG and is a manifestation of the general properties (132) and (133).

Second, the terms (277)-(283) are only those on the right-hand side of the two-loop RG equations that are relevant to the current. They are all proportional to the matrix elements $\left(\alpha_{\eta \sigma}^{+}\left|P^{\alpha_{\eta \sigma}}\right| \alpha_{\eta \sigma}^{-}\right)$as was anticipated in Sec. III C3. This is also a general property that holds in any loop order of the RG. In the noninteracting limit $U=0$, this implies that all two-loop (and higher loops) corrections relevant to the current vanish exactly [cf. Eq. (245)]. We emphasize that there are additional terms not listed in Eqs. (277)-(283) that are irrelevant to the current. These describe the two-loop renormalization of the $\zeta$ coefficient and involve factors $\left(\alpha_{\eta \sigma}^{-}\left|P^{\alpha_{\eta \sigma}}\right| \alpha_{\eta \sigma}^{+}\right)$and therefore do not vanish, even for $U=0$. However, the corrections to this coefficient beyond the two-loop order do contain the factors (246) and again vanish for $U=0$. See for more details Ref. 50.

\section{RESULTS}

In this section, we perform a detailed numerical investigation of the zero-temperature two-loop RT-RG equations $d \bar{L}^{0}(E) / d \Lambda=d \bar{L}^{0}(E) /\left.d \Lambda\right|_{\text {one loop }}+d \bar{L}^{0}(E) /\left.d \Lambda\right|_{\text {two loop }}$, where the right-hand sides are given by (226) and (273). We calculate the current as explained after Eq. (209). We focus on the dependence on the interaction $U$ and the magnetic field $B$ as a function of both the bias $V$ and the gate voltage $V_{g}=-\epsilon$. To clearly structure the discussion, we first summarize the central features of the calculated conductance as exemplified in Figs. 6(a) and 6(b) for zero and finite magnetic field $B$, respectively, and assess the limits of applicability.

\section{A. Overview and limits of applicability}

At zero magnetic field, the dominant features in Fig. 6(a) are the Coulomb-blockade diamonds defined by lines along which a single-electron tunneling resonance appears. In our calculated results, these $d I / d V$ peaks are broadened on the scale $\Gamma$ due to nonperturbative tunneling processes and have a peak height $e^{2} / h$, i.e., the quantum conductance. Going into either of the Coulomb-blockade regimes, where the charge is quantized to $N=0,1$, and 2 , the current decays nonexponentially due to higher-order tunneling (cotunneling and higher-order processes). At very small bias, however, the conductance shows a pronounced anomaly, but only in the $N=1$ regime, where the dot has an unpaired spin. We stress from the start that this should not be naively identified with the Kondo anomaly of the Anderson model: the correct description of the Kondo peak requires three-loop RT-RG corrections, which are beyond the scope of this work. ${ }^{27,65}$ To clarify in which regimes of voltages our results apply, we first discuss the linear conductance through the spin channel $\sigma: g_{\sigma}=\left(d I_{\sigma} / d V\right)_{V=0}$, in particular the consistency with the 



FIG. 6. (Color online) Zero-temperature nonlinear conductance $d I / d V$ vs bias $V$ and gate voltage $V_{g}=-\epsilon$ for strong interaction $U=30 \Gamma$ and (a) zero magnetic field $B=0$ and (b) finite field $B=$ $9 \Gamma$. Both figures are calculated in two-loop RG [Eqs. (226) and (273)] and are converged with respect to the number of nonequilibrium Matsubara axes (cf. Sec. III B1) using a sufficiently large bandwidth $D=10^{3} \Gamma$. The linear conductance in (b) shows good agreement with the Friedel sum rule (cf. Fig. 7).

Friedel sum rule

$$
g_{\sigma}=\frac{e^{2}}{h} \sin ^{2}\left(\pi\left\langle n_{\sigma}\right\rangle\right) .
$$

In Fig. 7, it is clearly seen that at zero field $B=0$ the conductance increasingly violates the Friedel sum rule between the two SET peaks for larger $U$. The violation becomes maximal at the particle-hole symmetry point: our result reaches $4 e^{2} / h$ instead of $2 e^{2} / h$. At best, in this regime our twoloop approach can be a starting point for further three-loop corrections containing the log-divergent Kondo corrections: However, it should be noted that the violation is finite, even at zero $T$ : The key observation in Fig. 7 is that beyond a magnetic field $B \sim \Gamma$, only a renormalized elastic cotunneling background remains and our results rapidly become consistent with the Friedel sum rule. Clearly, at voltages above $B \sim \Gamma$, the three-loop Kondo renormalization is expected to be



FIG. 7. (Color online) Linear conductance $d I /\left.d V\right|_{V=0}$ as function of the gate voltage $V_{g}$, obtained numerically for bias $V=$ $0.001 \Gamma$ for which the response was checked to be linear for all $V_{g}$. Our two-loop RT-RG results (black curves) are compared with the Friedel sum rule conductance $\sum_{\sigma} g_{\sigma}$ (red curves), which are obtained from Eq. (286) using the occupations $\left\langle n_{\sigma}\right\rangle$ calculated within our RG. Across panels (a)-(d), the interaction increases, $U / \Gamma=0.0,2.5,5.0,10.0$. Within each panel, the magnetic field is increased, $B / \Gamma=0.0,1.0,5.0,10.0$. Panel (a) for $U=0.0$ serves as a reference, numerically confirming the analytic result (253) that already in one-loop RG we attain the exact noninteracting result for the current.

negligible compared to the one- and two-loop corrections that we accounted for here. In all further analyses, the low-bias regime $V<\Gamma$ will thus be ignored for magnetic fields $B \lesssim \Gamma$. We do, however, show our results in this bias regime for two reasons: (i) knowing the behavior of the two-loop scheme is of interest as it presents a starting point for future three-loop calculations, and (ii) the behavior of the two-loop approach can be compared with that for other methods in this regime. We note, e.g., that for $U=2.5 \Gamma$, the violation of the Friedel sum rule is still rather modest, even at zero field. Our two-loop RG thus accounts nonperturbatively for the strong tunneling effects at zero temperature, covering the complete finite-bias stability diagram, where previous perturbative generalized master/kinetic equation approaches ${ }^{29,30}$ break down.

Based on the above, we expect that for a finite magnetic field $B \gtrsim \Gamma$, the two-loop RT-RG calculations reliably address transport features, illustrated in Fig. 6(b), at all applied voltages. Clearly, the SET resonance peaks have been split due the Zeeman effect. The zero-bias anomaly splits into two inelastic cotunneling resonances at finite bias $V \approx B$ (Zeeman excitations). A much smaller zero-bias anomaly remains, which should be ignored, as mentioned above. The above-mentioned features are of course known from previous studies and have been observed in many experiments. Our approach, however, includes renormalization effects of these basic transport signatures, which are nonperturbative in $\Gamma$ far from equilibrium. The following more detailed analysis, bearing the above restrictions in mind, indeed reveals several low-temperature renormalization effects that may be of experimental relevance. 

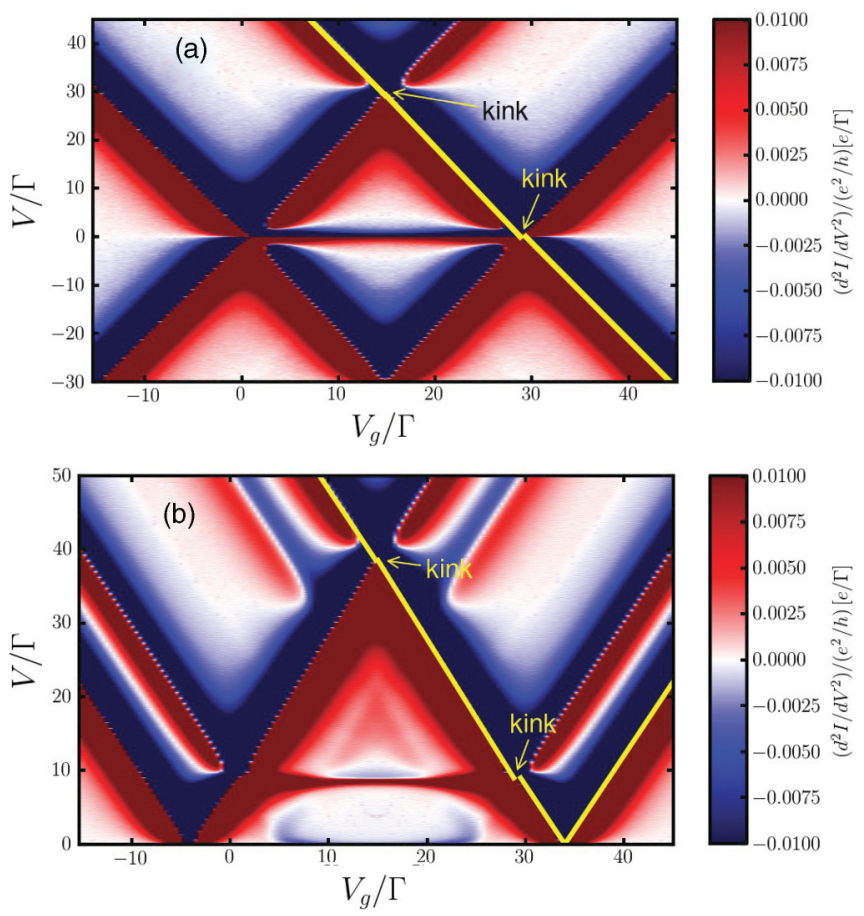

FIG. 8. (Color online) Peak positions of $d I / d V$ : shown is $d I^{2} / d V^{2}$ in a zoom of Fig. 6, making the zeros of $d I / d V$ stand out as curves separating red (positive) and blue (negative) regions. The yellow lines are guides to the eye obtained by accurate extrapolation of the linear parts of the resonance lines (including data points outside the figure). These emphasize the change of the slope of the linear parts of the resonance positions, in addition to the nonlinear renormalizations close to the kinks. In (a), kinks occur at $V \approx 0$ and $\pm U$, whereas in (b) they occur at $V \approx \pm B$ and $V= \pm U+B$. Note that in (b) there is no discernible kink at $V= \pm(U-B)$ : at this energy, there is a SET resonance "hitting" the Coulomb-blockade diamond edge but there is no onset of inelastic cotunneling, in contrast to $V= \pm B$, where there is such an excitation. This signals the importance of inelastic cotunneling for the appearance of such kinks.

\section{B. Single-electron resonance: Level renormalization and broadening}

\section{Kinks}

Careful inspection reveals that the SET resonance lines, in fact, change their slope when crossing $V=0$ and $U$. This can already be seen for small $\Gamma$ in Fig. 8(a), where we plot the $V$ derivative of Fig. 6(a) to follow the peak positions, adding a linear extrapolation. The SET resonance lines of the inner diamond change in such a way that the charge gap hardly renormalizes for $U \gg \Gamma$, although diamond distortions are visible by kinks in the linear extrapolations. The charge gap can both be determined from the height of the diamond (nonlinear response) or from the width of the diamond (linear response) and no significant deviation is found in this limit.

A related effect arises in a magnetic field: the SET slope below (above) the ICT threshold is smaller (larger) than the slope of the bare resonance line. As a result, the SET lines now show a kink at finite $V= \pm B$. We note that no such kink is seen at $V=U-B$ : this indicates that indeed the ICT is responsible for this effect since at $V=U-B$, in contrast to $V=B$ there is no ICT excitation. In (a) at $V=0$ there is some
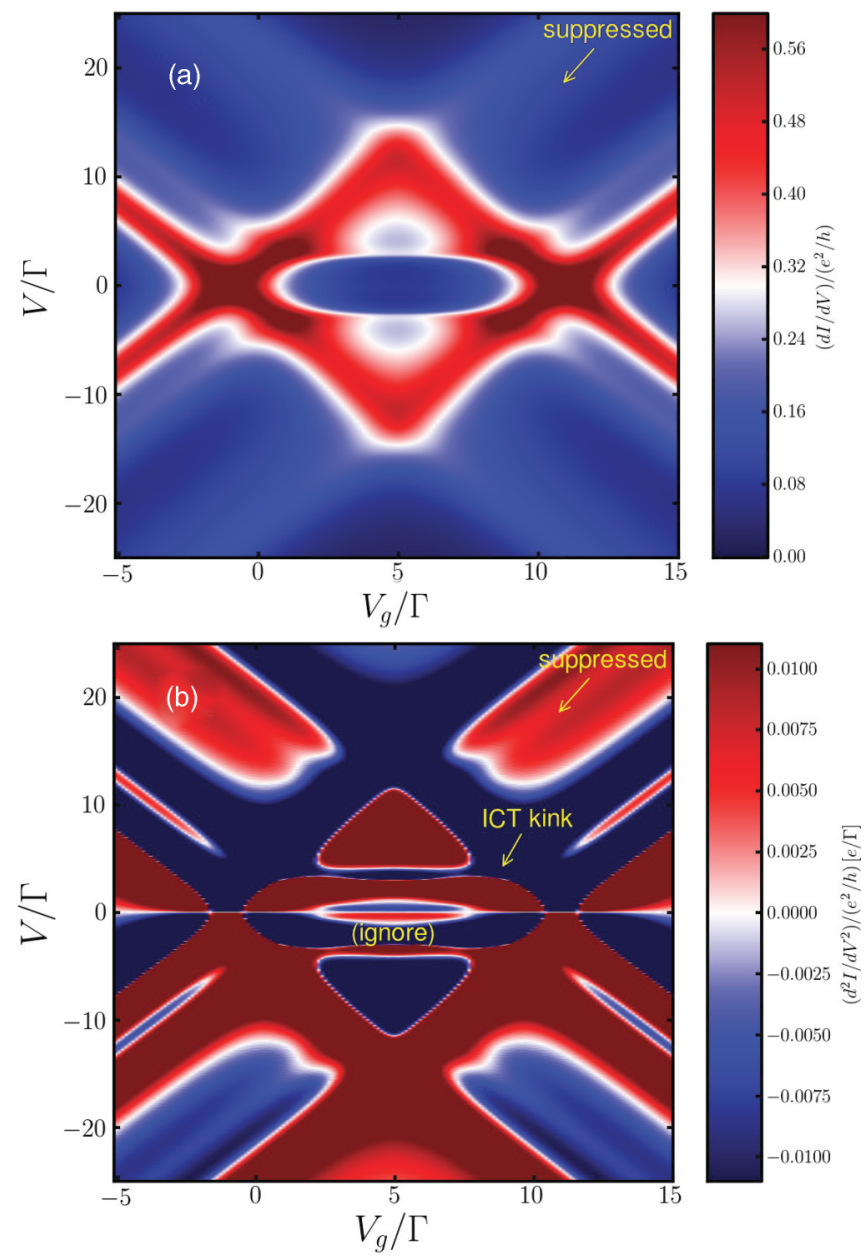

FIG. 9. (Color online) (a) Nonlinear conductance $d I / d V$ as in Fig. 6(b) but for reduced interaction $U=10 \Gamma$ and magnetic field $B=3 \Gamma$ and a symmetric bias range. (b) Derivative of (a), $d^{2} I / d V^{2}$, highlighting the renormalization of the SET peak position [see caption Fig. 8(b)], in particular near the onset of ICT.

nonlinearity around $V=\Gamma$ (small $V$ should be ignored, see above), which persists in (b) around $V=B$ in a magnetic field.

Upon increasing $\Gamma$ relative to $U$, these effects are enhanced as shown in Fig. 9(a). The edges of the $N=1$ Coulombblockade regime tend to bend inwards, towards the diamond center. Notably, above the onset of ICT, the slope is slightly larger than that of the bare resonance line. We furthermore observe that this also leads to the suppression of the excitation $|\downarrow\rangle \rightarrow|2\rangle$ at $\mu_{L}-\mu_{R}=\epsilon_{\uparrow}+U$ in Fig. 9 (see arrow), which is still clearly visible in Fig. 6 [see arrows in Figs. 9(a) and 9(b)].

\section{Analysis}

It seems not possible to analytically extract a simple physical picture explaining the above nonlinearities. The following analysis aims to indicate why this is the case: we trace back at which stage of the two-loop RG scheme the various effects are generated, taking the parameter set of Fig. 9(b) as a starting point. In Fig. 10, we show the conductance calculated both in one- and two-loop RG, both 


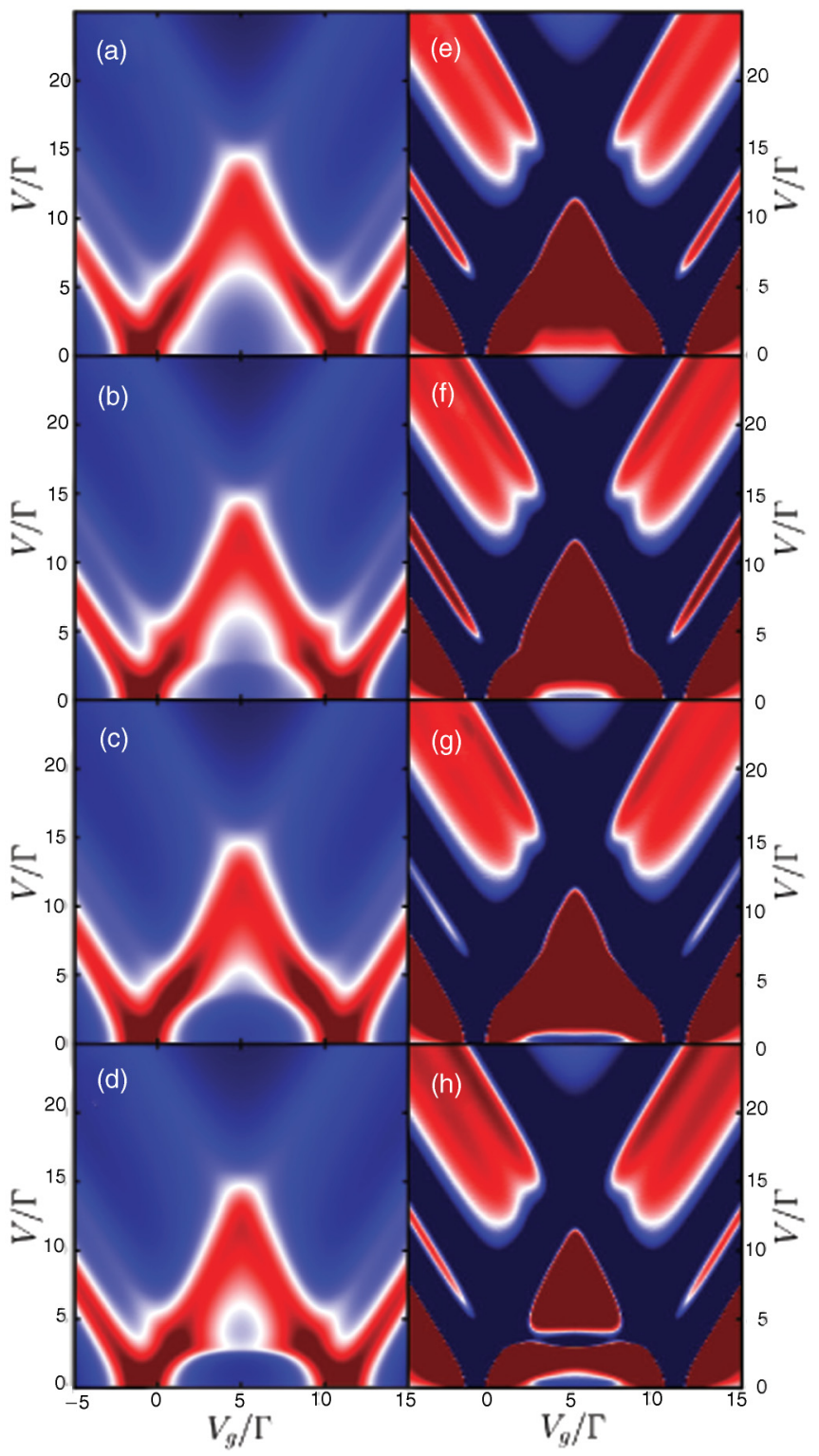

FIG. 10. (Color online) Comparison of $d I / d V$ (same color scale as Fig. 9) calculated in the one-loop [(a), (b)] and two-loop approximations [(c), (d)], neglecting all Matsubara axes [(a), (c)] and fully converging using 15 frequency axes [(b), (d)]. The second column shows the corresponding $d I^{2} / d V^{2}$ maps, allowing the $d I / d V$ peak positions to be followed [see caption Fig. 8(b)]. In particular, the inelastic cotunneling excitation at $V=B$ evolves from a step in (a)-(c) into a peak in (d). Clearly, the nonequilibrium Matsubara frequency dependence is responsible for the "kinks" in the SET resonance (they already appear in one loop with quantitative modifications in two loop).

with and without converging the calculations with respect to the nonequilibrium Matsubara axes.

Clearly, the different slopes and nonlinearities already arise in the one-loop RG: this is visible from Figs. 10(b) and 10(f). Their strength correlates with that of the signatures of ICT appearing in the stability map in the various approximations. However, this effect only arises when the Matsubara axes are accounted for: this is seen by directly comparing Figs. 10(a)

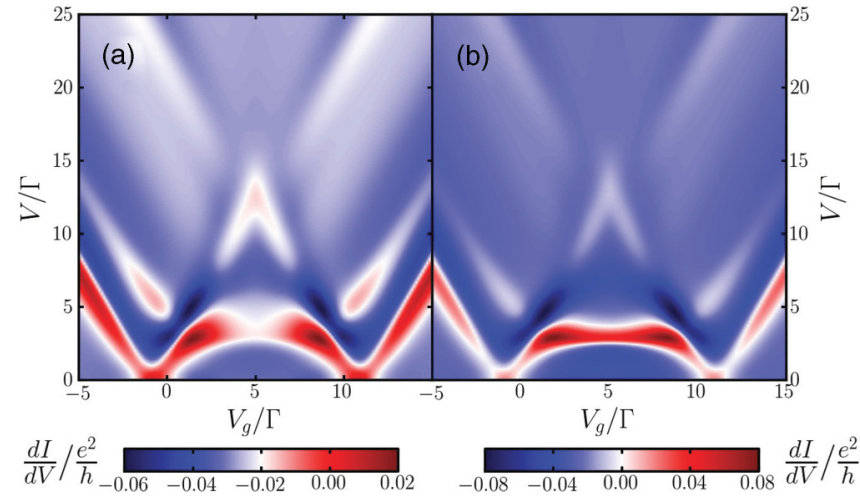

FIG. 11. (Color online) Effect of Matsubara axes convergence, i.e, the converged result minus the result neglecting all Matsubara axes: (a) one-loop RG [Figs. 10(b) and 10(a)] and (b) two-loop RG [Figs. 10(d) and 10(c)]. Adjacent red and dark blue regions indicate that the correction is an S-shaped curve, which, when added to a peaked curve, results in a shift of the peak position. Clearly, the Matsubara frequency dependence has an impact on the positions of all resonances and should be fully accounted for.

and 10 (b) and is confirmed by Fig. 11, where we explicitly plot the difference of former two figures.

Overall, the two-loop corrections are most pronounced along the SET-regime boundaries and the ICT threshold as comparison of Figs. 10(b) and 10(d) and the plot of their difference in Fig. 12 shows. Also, in two-loop order, the kinks are most pronounced in the Matsubara-converged result. We conclude that the two-loop fluctuation effects result in a nontrivial energy dependence of the vertices and Liouvillian, which shows up in anomalous features of the measurable stability diagram, even for such a simple Anderson model of a quantum dot. We emphasize that these features are not related to renormalization processes that generate the Kondo effect (three loop, not included here) and have an effect at $V \sim B \gtrsim \Gamma$.

\section{Experimental implications}

Having traced the origin of the change of the slopes and the nonlinearities of the SET resonances, we now discuss their

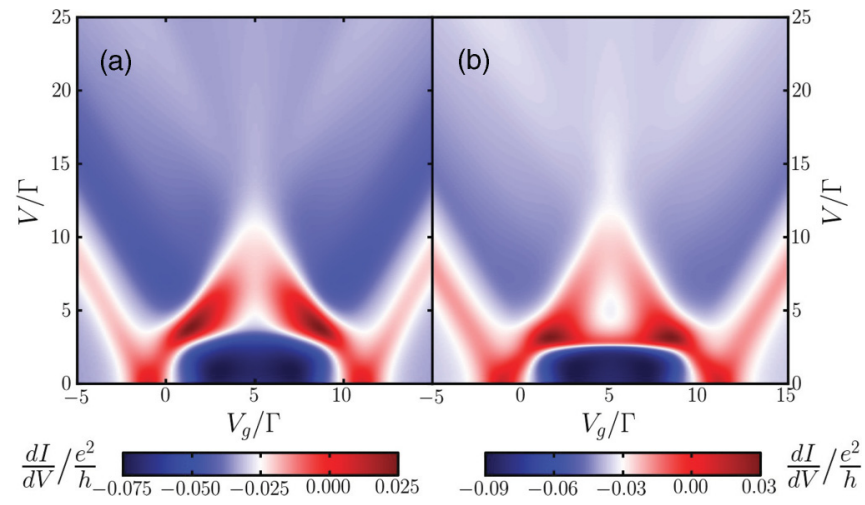

FIG. 12. (Color online) Effect of two-loop corrections, i.e., the two-loop result minus the one-loop result (a) without Matsubara frequency dependence [Figs. 10(c)-10(a)]; (b) with converged Matsubara frequency dependence [Figs. 10(d)-10(b)]. Note the positive corrections to the magnitude of the inelastic cotunneling in (b). 

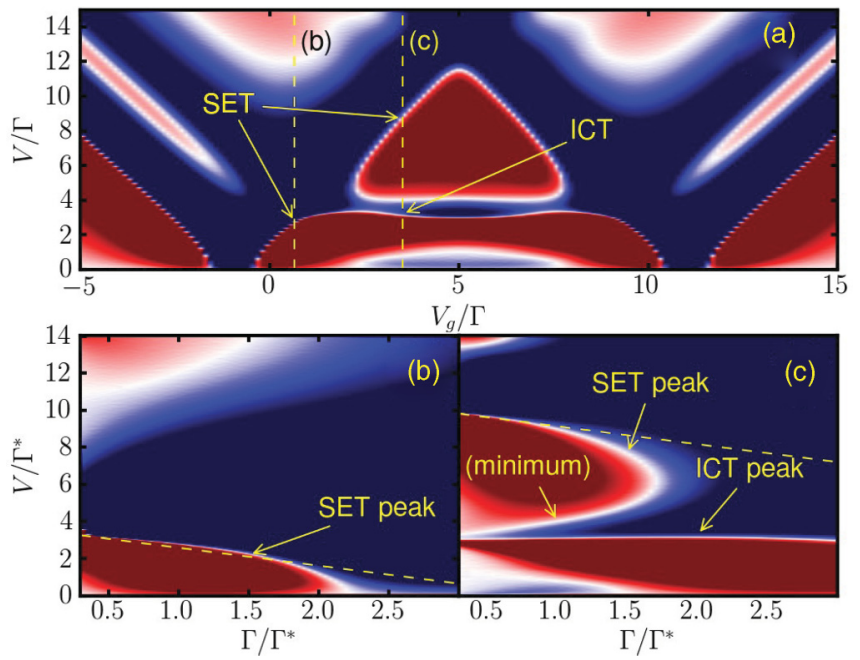

FIG. 13. (Color online) Distortion of the stability diagram with increasing tunnel coupling $\Gamma$. (a) Zoom-in of Fig. 9 of $d I^{2} / d V^{2}$ (same scales and units), highlighting the gate-voltage dependence of the SET and ICT bias thresholds generated by tunnel renormalization. Here, $U=10.0 \Gamma^{*}, B=3.0 \Gamma^{*}$, and $\Gamma^{*}$ is the reference value of $\Gamma$. In panels (b) and (c), we show the evolution of the zeros of $d I^{2} / d V^{2}$ as the tunnel coupling is increased for fixed $U$ and $B$. Here, $\Gamma$ varies from $0.3 \Gamma^{*}$ to $3.0 \Gamma^{*}$ along a fixed gate-voltage cut in (a), marked the vertical dashed line $V_{g}=-\epsilon=0.65 \Gamma^{*}$ for (b), and $V_{g}=-\epsilon=$ $3.50 \Gamma^{*}$ for (c). The dashed linear approximation to the renormalized SET positions in (c) is copied with a vertical offset to (b), showing that the renormalization is nonuniform in the gate voltage. This signals a distorted stability diagram. The ICT peak clearly has a weaker $\Gamma$ dependence which, moreover, can be seen to be nonmonotonic when calculated for a larger $\Gamma$ range.

relevance to experiments. In fact, kinks in SET resonances are often observed in various types of quantum dot systems. ${ }^{66-69}$ Our calculations indicate that tunnel-induced renormalization is a possible mechanism for their occurrence, but other (e.g., electrostatic) mechanisms ${ }^{70,71}$ should not be ruled out in an experimental situation. However, for strong coupling, it is physically not unexpected that when ICT sets on, the level renormalization significantly changes, resulting in such a kink.

A direct test of this assumption would be to track the Coulomb diamond as a function of the coupling strength $\Gamma$. In Figs. 13(b) and 13(c), we show predictions for the evolution of the SET resonance point for two fixed gate voltages, one below and one above the ICT threshold, respectively. The main observation from such a plot is that the peaks evolve along curves that are not simply offset by a constant bias. This indicates that the renormalization of SET resonance becomes increasingly nonlinear and a kink must develop. (Note that experimentally $\Gamma$ may change nonlinearly with control voltages, but this does not spoil the argument.) It is important to properly choose the point above the ICT threshold: depending on the gate-voltage position, the peak may renormalize stronger or weaker than the peak below the threshold. We are aware that experimentally such tuning of $\Gamma$ with gate voltages may lead to other side effects that may be hard to distinguish from the effect. Here, the different renormalization of the ICT resonance can be of use, which is discussed next.

\section{Cotunneling resonance: Gap renormalization and reduced broadening}

Having discussed the effect of the ICT on the SET resonances, we now study the ICT features themselves in more detail. Figure 14 shows how the inelastic cotunneling resonance exhibits a Zeeman splitting with increasing magnetic field. Despite the zero temperature, the width of the inelastic cotunneling feature is finite and can be clearly seen to depend on the magnetic field, and thereby on the voltage at which this resonance occurs. This is in contrast to high-temperature two-loop perturbation theory ${ }^{29,30}$ where this resonance appears as a thermally broadened feature at the unrenormalized excitation energy. At larger voltage $V=B$, the resonance width increases, reflecting a decreasing lifetime. This energy dependence of the width is generated by our two-loop renormalization since the initial Liouvillian of the RG flow [Eq. (135)] has imaginary parts that are all $\sim \Gamma$.

Next, as the magnetic field is reduced, but still on the order of several times $\Gamma$, the differential conductance develops a pronounced peak on top of the inelastic tunneling step: in Fig. 14(b), this is signaled by the onset of a negative second derivative of the current (blue) and is clearly seen in the conductance traces in Fig. 14(c). It is known that part of such a peak on top of the well-known inelastic tunneling step $\mathrm{p}^{72}$ is due to nonequilibrium occupations ${ }^{30,73-75}$ that we also fully take into account. The enhanced conductance at the cotunneling resonance is due to the two-loop renormalization, including the frequency dependence. Only after including both two-loop corrections and converging with respect to the Matsubara frequency axes, the ICT resonance evolves from a $d I / d V$ step into a peak. This was illustrated (for smaller $U$ ) in Fig. 10: (a)-(c) show no ICT peak [since their derivatives (e)-(g) have no zero at the ICT threshold $V \approx B$ ] in contrast to Fig. $10(\mathrm{~d})$ (see also Fig. 13).

This enhancement is not related to Kondo-exchange tunneling, which is known to lead to additional logarithmic enhancements: ${ }^{27,74}$ their renormalization is not included in our two-loop calculations and is expected to be of limited importance at this large magnetic field (several times $\Gamma$ ). The qualitative change of the inelastic conductance feature from a step to a peak in Fig. 14(b) implies some ambiguity in the extraction of the excitation energy, either from the inflection point of the step (upper right corner) or from the peak position (lower left corner).

Finally, close inspection Fig. 13(a) shows that the ICT bias peak position has a weak gate-voltage dependence. In contrast, in the high-temperature limit, this resonance lies at the unrenormalized cotunneling excitation $V=B$. Strong tunneling thus leads to an apparent renormalization in the cotunneling peak position, even for this simple model (cf. Ref. 76). Although our effective Liouvillian contains parameters $E_{\sigma}$ and $F_{\eta \sigma}$ that relate to the magnetic field [compare Eqs. (135) and (136)], these parameters depend on energy, and our full result requires their values at several different energy scales since many nonequilibrium Matsubara frequency axes must be accounted for. 

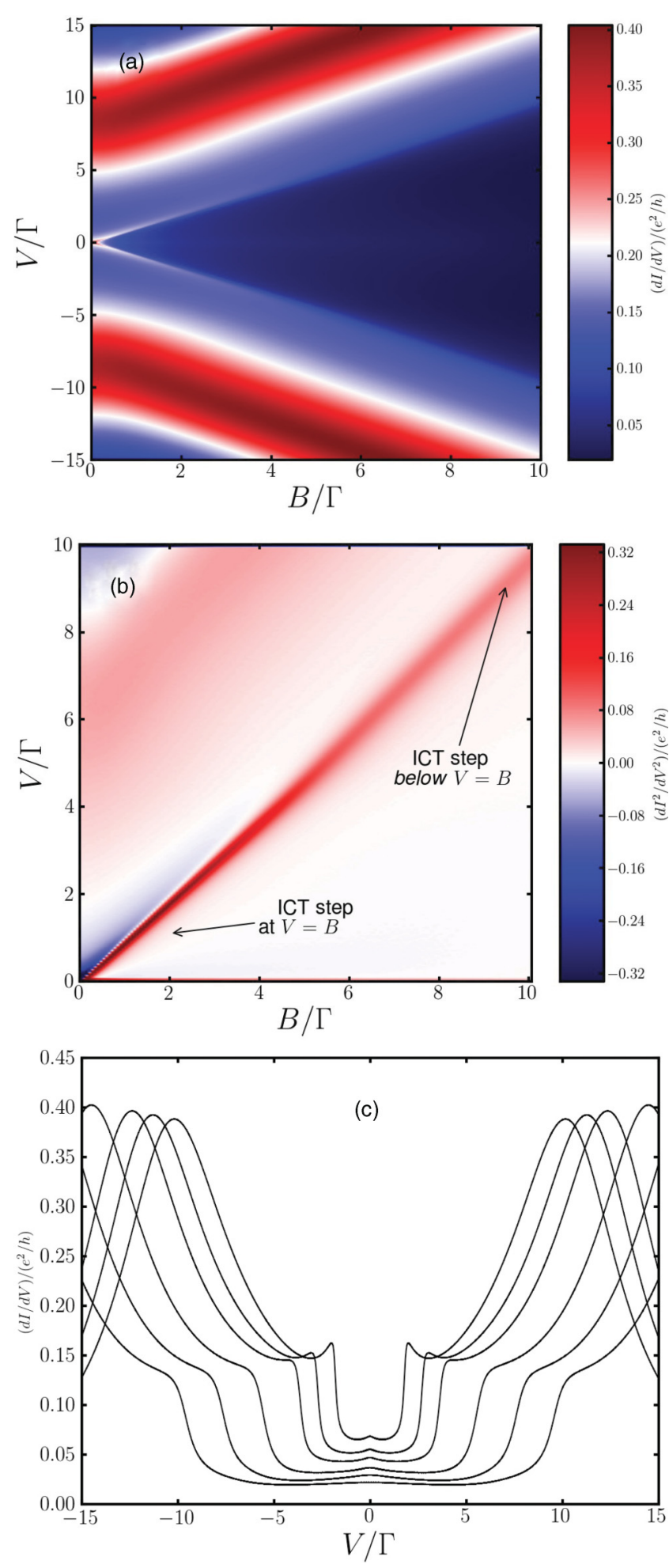

FIG. 14. (Color online) (a) Zeeman splitting of the cotunneling resonance (change in blue color) and shift of the SET peaks (red peak) for $U=30 \Gamma$ and $V_{g}=-\epsilon=U / 6=5 \Gamma$. (b) Zoom-in of the bias derivative of (a), i.e., $d I^{2} / d V^{2}$ showing the resonance position. The sharp peak in $d I / d V$ in (a), starting from the lower left corner, closely follows the diagonal line $V=B$. The step in $d I / d V$ in (a), starting from the upper right corner, instead follows a line that is parallel to the diagonal $V=B$ but offset by a constant, negative magnetic field (in this case $\approx 0.45 \Gamma$ ). (c) $d I / d V$ traces of (a) for $B / \Gamma=2.0,3.0,4.0,6.0,8.0,10.0$.

\section{OVERVIEW AND OUTLOOK}

We have studied the standard model of an interacting quantum dot, an Anderson impurity, in the low-temperature nonequilibrium limit. We have calculated the effective timeevolution kernel $L(z)=L+\Sigma(z)$ for the kinetic equation of the reduced density operator, where $\Sigma$ is the nontrivial self-energy superoperator. In contrast to many previous studies using such a generalized/quantum master equation approach, we have calculated the time-evolution kernel using the realtime renormalization group ( $R G)$. The equations for the effective kernel are integrated as a function of a cutoff parameter $\Lambda$ : as $\Lambda$ is reduced, the renormalized Liouvillian $\bar{L}_{\Lambda}$ in principle flows to the exact result $\left.\bar{L}_{\Lambda}\right|_{\Lambda=0}=L(z)$. This $\mathrm{RG}$ calculation of the effective kernel involves a number of key elements:

(i) Transitions between all Liouville space supervectors need to be accounted for. This includes those elements of $\bar{L}_{\Lambda}$ and $L(z)$, which in a perturbative calculation of the kernel drop out due to conservation laws (charge, spin, and possibly particle-hole symmetry). The reason for this is that as one integrates out energy scales, effectively higher-order diagrams are included into a renormalization of the kernel that describe virtual intermediate states, which are less restricted by conservation laws.

(ii) The dependence of the renormalized kernel on the real $Q D$ frequency $(E)$ (Laplace variable conjugate to time) is important even in the stationary state. During the RG flow, the renormalized Liouvillian at frequency $E$ self-consistently couples to its action on virtual intermediate states at frequencies differing from $E$ by multiples of the bias voltage $\mu_{L}-\mu_{R}$. We have shown that this nonequilibrium effect leads to significant quantitative corrections and may require tens of bias multiples to achieve convergence.

(iii) The reservoir-frequency $(i \omega)$ dependence of both the kernel and the vertices becomes important when going beyond the leading, one-loop approximation, in addition to the QD frequency $E$. This dependence is generated on the imaginary frequency axis when the reservoirs are integrated out and it may cancel two-loop corrections calculated without frequency dependence.

We have systematically accounted for the leading frequency correction within a one-plus-two-loop approximation to the exact RT-RG equations and derived an effective RG equation for the time-evolution kernel only. This includes the relevant vertex renormalization corrections. Importantly, the leading frequency correction of the one-loop renormalization of the Liouvillian $\bar{L}_{\Lambda}$ was found to exactly cancel the two-loop zero frequency term.

For the noninteracting Anderson problem $(U=0)$, we found that the current is exactly captured already in the one-loop approximation without any frequency dependence even though the complete solution (i.e., including the density operator) is contained only within the one-plus-two-loop approximation. For the strongly interacting case of interest, the nonlinear transport spectrum $(d I / d V$ stability diagram) was calculated for a wide range of parameters. The different, intrinsic broadening of the single-electron tunneling and cotunneling resonances was captured, as well as the zerotemperature renormalization of their positions. As emphasized 
throughout, due to the restriction to one- and two-loop diagrams, the small Kondo regime can not be addressed. This regime has been recently studied in detail using the RT-RG approach based on the mapping to a Kondo model. This allowed the entire crossover from weak to strong coupling to be described. ${ }^{65}$ Our study thus provides a starting point for a three-loop analysis of the nonequilibrium Anderson model in which the interplay of Kondo spin fluctuations and charge fluctuations can be described.

The RT-RG study benefited a lot from an extensive reformulation of the underlying real-time perturbation theory in terms of vertex superoperators $\bar{G}$ and $\tilde{G}$. Although originally introduced in the context of the RT-RG ${ }^{26}$ we have revealed their full significance as fermionic field superoperators that directly generate the Liouville Fock space in complete analogy to closed quantum many-body systems:

(a) Field superoperators obey definite anticommutation relations, and a simple fluctuation-dissipation relation similar to that of the underlying usual field operators. The Wick theorem in Liouville space at finite temperature can be obtained algebraically using relation (59) in the usual way, ${ }^{77}$ avoiding the necessary careful explicit accounting of fermion-parity signs in other proofs. ${ }^{26}$ This difficult aspect of Liouville space fields was noted earlier. ${ }^{34,44}$

(b) The causal structure of the theory is reflected by the vanishing of two out of four reservoir correlation functions (rather than one out of four, as in the Keldysh Green's-function technique). This results in an exponential reduction of the number of terms contributing to the time-evolution kernel (additional to the reduction in the wide-band limit). The remaining contributing diagrams are easily identified by their topology.

We have extended the use of these fermionic field superoperators to the perturbation theory underlying the RT-RG. This resulting causal representation of the perturbation theory has many advantages:

(i) Probability conservation of the kernel is manifest termby-term, allowing nonconserving approximations to be easily spotted. In addition, other exact eigenvectors and eigenvalues of the kernel were found, which limit the form of the exact effective Liouvillian $L(z)$ as expressed in our central result Eq. (136).

(ii) Term-by-term diagrammatic evaluation of the wideband limit. Diagrams that vanish in this limit can be identified by their topology, and the remaining diagrams can be shown to be independent of the cutoff using the fundamental fermionic algebra of the field superoperators. This results in a further strong reduction in the number of contributing terms as a function of the perturbation order. Moreover, the advantage of working with the complete space of the QD states becomes explicit.

(iii) Finally, the fundamental importance of the infinitetemperature limit becomes explicit. It defines the LiouvilleFock-space vacuum, and the perturbation theory can be explicitly decomposed into infinite-temperature renormalization effects and the nontrivial finite-temperature contributions. This provides a natural starting point for the RT-RG, which readily suggests itself.

Aside from their application to the RT-RG, we have already found useful application of some of these points in perturbative studies, as discussed here in connection with Ref. 39 and in forthcoming works on time dependence ${ }^{50}$ and adiabatic driving. ${ }^{38}$

\section{ACKNOWLEDGMENTS}

This work was initiated by intense discussions with H. Schoeller, which are gratefully acknowledged. We thank D. DiVincenzo, T. Costi, and J. Splettstoesser for valuable comments and A. Flesch, E. Gorelov, and F. Reckermann for suggestions regarding the numerical calculations. We are grateful to D. Kosov for pointing out Prosen's work and his kind help in establishing the relations between our and other existing superfermion approaches. This work has also benefited from a preliminary study undertaken together with M. Kurz.

\section{APPENDIX A: WICK THEOREM FOR CAUSAL FIELD SUPEROPERATORS OF THE RESERVOIRS $J^{q}$}

Here, we show how the algebraic proof of the Wick theorem ${ }^{77}$ for standard field operators directly applies to the field superoperators $J$ in the causal representation. In this proof, one considers the average of $n$ reservoir field superoperators $J_{i}^{q_{i}}$ defined by Eq. (52),

$$
X=\operatorname{Tr}_{\mathrm{R}}\left(J_{1}^{q_{1}} \ldots J_{n}^{q_{n}} \rho^{R}\right),
$$

which can be nonzero only for even $n$. One then commutes $J_{n}^{q_{n}}$ to the left-hand side, using that the field superoperators obey the anticommutation relations (54). We first consider the case $q_{n}=-$ and make use of the zero-trace property (62) of the causal representation. This reduces the average to expressions of the same form

$$
X=\sum_{k \neq n}(-1)^{N_{k, n}} \tilde{\gamma}_{k, n} X_{k, n},
$$

but with averages over $n-2$ operators

$$
X_{n, k}=\underset{\mathrm{R}}{\operatorname{Tr}}\left(J_{1}^{q_{1}} \ldots J_{k-1}^{q_{k-1}} J_{k+1}^{q_{k+1}} \ldots J_{n-1}^{q_{n-1}} \rho_{\text {res }}\right),
$$

weighted with the contraction function

$$
\tilde{\gamma}_{k, n}=\frac{\Gamma_{0}}{2 \pi} \delta_{k, \bar{n}} \delta_{+, q_{k}}
$$

Here, $N_{k, n}=n-k-1$ is the number of permutations to bring $J_{k}^{q_{k}}$ and $J_{n}^{q_{n}}$ together.

For $q_{n}=+$, we proceed in the same way, except that before permuting $J_{n}^{q_{n}}$ to the far left, we apply the fluctuationdissipation superoperator identity (59). This transforms the expression into that for the $q_{n}=-$ case multiplied with the Keldysh distribution function: Eq. (A2) applies again but with $\tilde{\gamma}_{k, n}$ replaced by

$$
\bar{\gamma}_{k, n}=\tanh \left(\eta_{n} \omega_{n} / 2 T_{r_{n}}\right) \tilde{\gamma}_{k, n},
$$

where the multi-index $n$ of $J_{n}^{q_{n}}$ reads as $n=\eta_{n}, \sigma_{n}, r_{n}, \omega_{n}$.

We thus find the standard Wick recursion relation

$$
X=\sum_{k \neq n}(-1)^{N_{k, n}} \gamma_{k, n} X_{k, n}
$$

with $\gamma_{k, n}=\bar{\gamma}_{k, n} \delta_{+, q_{n}}+\tilde{\gamma}_{k, n} \delta_{-, q_{n}}$, which by iteration gives the Wick theorem (60) with the contractions (63) and (64). 


\section{APPENDIX B: MAIN PROPERTIES OF THE CAUSAL VERTEX SUPEROPERATORS $G^{q}$}

"Bare" vertices. In Sec. II B2, the main properties (53) and (57) of the causal superoperators $\bar{G}$ and $\tilde{G}$, respectively, were introduced, namely, their anticommutation relations (53) and their relation by Hermitian conjugation in Liouville space [Eq. (57)]. We now give the proof of the latter relation. We note that both relations are fundamental as they imply a formal correspondence of causal $G^{ \pm}=\bar{G}, \tilde{G}$ operators with the usual fermionic field operators and allow us to develop the "second quantization" technique for fermionic Liouville Fock space.

Super-Hermitian conjugation is defined relative to the scalar product in Liouville space, $(A \mid B)=\operatorname{Tr} A^{\dagger} B$, where $A$ and $B$ are dot operators. To prove Eq. (57), we first notice that the "naive" field superoperators (40) obey

$$
\left(\mathscr{G}_{1}^{p}\right)^{\dagger}=\mathscr{G}_{\overline{1}}^{p},
$$

where $1=\eta \sigma$ and $\overline{1}=\bar{\eta} \sigma$. Superoperators with the same Keldysh index $p$ are thus conjugated to each other in the usual way: Hermitian conjugation is equivalent to inverting the particle-hole index $\eta$. This follows from the cyclic property of the trace:

$$
\begin{gathered}
\left(A\left|\mathscr{G}_{1}^{+}\right| B\right)=\operatorname{Tr}_{D} A^{\dagger} d_{\eta, \sigma} B=\left(\operatorname{Tr}_{D} B^{\dagger} d_{\bar{\eta}, \sigma} A\right)^{*} \\
=\left(B\left|\mathscr{G}_{\overline{1}}^{+}\right| A\right)^{*}=\left(B\left|\left(\mathscr{G}_{1}^{+}\right)^{\dagger}\right| A\right)^{*}, \\
\left(A\left|\mathscr{G}_{1}^{-}\right| B\right)=\operatorname{Tr}_{D} A^{\dagger} B d_{\eta, \sigma}=\left(\operatorname{Tr}_{D} d_{\bar{\eta}, \sigma} B^{\dagger} A\right)^{*}=\left(\operatorname{Tr}_{D} B^{\dagger} A d_{\bar{\eta}, \sigma}\right)^{*} \\
=\left(B\left|\mathscr{G}_{\overline{1}}^{-}\right| A\right)^{*}=\left(B\left|\left(\mathscr{G}_{1}^{-}\right)^{\dagger}\right| A\right)^{*},
\end{gathered}
$$

where $*$ denotes complex conjugation and $\dagger$ denotes either usual Hermitian conjugation or super-Hermitian conjugation depending on whether it acts on an operator or superoperator, respectively. These superoperators, however, have the disadvantage that they satisfy no definite fermionic or bosonic commutation relations [cf. Eq. (45)].

The transformed field superoperators (47), which include the fermion-parity sign, obey the definite anticommutation relations (49). However, they are not related by super-Hermitian conjugation in the usual way:

$$
\left(\mathcal{G}_{1}^{p}\right)^{\dagger}=p \mathcal{G}_{\overline{1}}^{p},
$$

which follows from Eq. (B1) and the properties $\left(L^{n}\right)^{\dagger}=L^{n}$ and $\left[L^{n}, \mathscr{G}_{1}^{p}\right]_{-}=\eta \mathscr{G}_{1}^{p}$ of $L^{n}=[n, \bullet]_{-}$:

$$
\left(\mathcal{G}_{1}^{p}\right)^{\dagger}=\left(p^{L^{n}} \mathscr{G}_{1}^{p}\right)^{\dagger}=\left(\mathscr{G}_{1}^{p}\right)^{\dagger} p^{L^{n}}=p^{L^{n}+1} \mathscr{G}_{\overline{1}}^{p}=p \mathcal{G}_{\overline{1}}^{p} .
$$

Finally, the causal field superoperators (51) obtained from $\mathcal{G}^{p}$ by a Keldysh rotation obey both definite commutation relations (53) and standard Hermitian-superconjugation relations (57): using $p^{2}=1$, we obtain

$$
\begin{aligned}
\left(G_{1}^{q}\right)^{\dagger} & =\frac{1}{\sqrt{2}} \sum_{p} p^{(1-q) / 2}\left(\mathcal{G}_{1}^{p}\right)^{\dagger} \\
& =\frac{1}{\sqrt{2}} \sum_{p} p^{(3-q) / 2} \mathcal{G}_{\overline{1}}^{p}=G_{\overline{1}}^{\bar{q}} .
\end{aligned}
$$

This clearly demonstrates the fundamental advantage of the causal representation over the other ones.
Note that due to the $p$ dependence of the superoperators entering into the definition (51) of our superoperator $\bar{G}_{\eta \sigma}$, the latter is either a commutator or an anticommutator with the fermionic operator $d_{\eta, \sigma}$, depending on the argument on which it acts. For example, $\bar{G}_{\eta \sigma} A=\left[d_{\eta, \sigma}, A\right]_{-}$if $A$ is a fermionic dot operator [odd in charge, see Eq. (96)] and $\bar{G}_{\eta \sigma} A=\left[d_{\eta, \sigma}, A\right]_{+}$if $A$ is bosonic [even in charge, see Eq. (91)-(95)]. For the $\tilde{G}_{\eta \sigma}$, the opposite holds. The crucial relation $(55), \operatorname{Tr}_{D} \bar{G}_{\eta \sigma}=0$, nevertheless always holds: one obtains zero in the first case as the trace of a commutator and in the second case as the trace of an operator which is off diagonal in charge.

The analogy to the usual field operators extends also to the transformation behavior under spin rotations. The usual field operators in Fock space, $d_{\sigma}^{\dagger}$ and $\sigma d_{\bar{\sigma}}$, transform as irreducible tensor operators (ITOs) of rank $\frac{1}{2}$ and index $\sigma / 2$ (note the $\sigma$ signs). Similarly, superoperators corresponding to particle fields $(\eta=+) G_{+\sigma}^{q}$ and hole fields $(\eta=-) \sigma G_{-\bar{\sigma}}^{q}$ are irreducible tensor superoperators (ITSOs) of rank $\frac{1}{2}$ with index $\sigma / 2$. This applies to all the representations of the field superoperators that we used: the same holds for $\mathcal{G}_{+\sigma}^{p}, \sigma \mathcal{G}_{-\bar{\sigma}}^{p}$ and $\mathscr{G}_{+\sigma}^{p}, \sigma \mathscr{G}_{-\bar{\sigma}}^{p}$.

To prove this, we note that for any two superoperators $A^{p}$ and $B^{p^{\prime}}$ generated in the same way as $G_{1}^{p}$ [cf. Eq. (40)] by two operators $A$ and $B$,

$$
A^{p} \bullet= \begin{cases}A \bullet & p=+, \\ \bullet A & p=-,\end{cases}
$$

the commutator of superoperators can be expressed in the superoperator corresponding to the commutator: $\left[A^{p}, B^{p^{\prime}}\right]_{-}=$ $p \delta_{p, p^{\prime}}\left([A, B]_{-}\right)^{p}$. This directly shows that the field superoperators $\mathscr{G}_{1}^{p}$ (40) transform in the same way as the field operators $d_{1}$ under spin rotations: $\left[L^{S_{i}}, \mathscr{G}_{1}^{p}\right]_{-}=\left(\left[S_{i}, d_{1}\right]_{-}\right)^{p}$. The superoperators (47) and (51) simply inherit this property since spin and charge superoperators commute $\left[L^{S_{i}}, L^{n}\right]_{-}=0$.

\section{APPENDIX C: PROSEN'S FERMIONIC SUPEROPERATORS}

For open systems described by a Lindblad equation that is quadratic in fermion operators, superoperator fields similar to ours [cf. Eq. (51)] were introduced by Prosen ${ }^{31}$ with the aim of explicitly constructing the steady state in the Liouville Fock space. These fields obey the anticommutation relations (53) and the Hermitian-conjugation relations (57). Moreover, the superoperators $c_{j}^{\dagger}$ introduced in that work also satisfy Eq. (55) and thus possess the causal structure discussed here. The latter property is, however, not explicitly seen in the formulation of Ref. 31, and becomes clear only after setting up the correspondence (C1) between our, and Prosen's, superoperators.

In Ref. 31, the opposite order of construction was used, i.e., first a Fock space was constructed [cf. our Eqs. (91)-(96)] as an ordered product of the Majorana operators, which are linear combinations of usual fermion creation and annihilation operators. Then, fermionic superoperators were defined on this space [cf. our Eq. (51)]. The fermion-parity superselection rule, which plays an explicit, key role in our construction, was implicitly taken into account by the ordering of the Majorana operators and by a specific definition of the creation and annihilation superoperators. Careful comparison of the Fock 
spaces shows that superoperators $c_{j}^{\dagger}$ of Ref. 31 are related to our $\bar{G}_{\eta, \sigma}$ by the following unitary transformation:

$$
\begin{array}{ll}
c_{j}^{\dagger}=\frac{1}{\sqrt{2}}\left(\bar{G}_{+, m}^{\prime}+\bar{G}_{-, m}^{\prime}\right), \quad j=2 m-1 \\
c_{j}^{\dagger}=\frac{i}{\sqrt{2}}\left(\bar{G}_{-, m}^{\prime}-\bar{G}_{+, m}^{\prime}\right), & j=2 m .
\end{array}
$$

Corresponding relations for the superoperators $c_{j}$ can be obtained from Eq. (C1) by super-Hermitian conjugation. Here, $m=1,2,3, \ldots$ enumerates the fermionic channels and $\bar{G}_{\eta, m}^{\prime}$ are the $\bar{G}_{1}$ superoperators renumbered by the channel index. For our single-level Anderson model, we have only spin channels, giving

$$
\bar{G}_{\eta, m}^{\prime} \equiv\left\{\begin{array}{l}
\bar{G}_{\eta, \downarrow}, m=1 \\
\bar{G}_{\eta, \uparrow}, m=2 .
\end{array}\right.
$$

For a multilevel model with several discrete channels numbered $k=1,2,3, \ldots$,

$$
\bar{G}_{\eta, m}^{\prime}:=\bar{G}_{\eta, \sigma}^{k}, \quad m=2 k+(\sigma-1) / 2 .
$$

It is not directly clear how the definitions in Ref. 31 can be applied to the case of an infinite and, especially, a continuous number of channels. In fact, the relation (C1) can be used as a definition of the $c_{j}, c_{j}^{\dagger}$ superoperators in this case since the superoperators $\bar{G}_{1}, \tilde{G}_{1}$ have a proper definition also in this limit [see Eq. (54)]. In contrast to our case, the superoperators $c_{j}^{\dagger}$ are not irreducible tensor superoperators (ITSOs) with respect to either spin or charge rotations. This fact would complicate the symmetry classification similar to that performed in Sec. II C3. Moreover, the causal structure of the kernel, which in general plays a crucial role as our analysis shows, also remains implicit in the representation. ${ }^{31}$ Finally, we emphasize the different scope of applications of the field superoperators in our work: Whereas Prosen's approach was formulated to calculate steady states of quadratic effective Louvillians, we here extend it to the reservoirs with continuous fields and use it to simplify the microscopic derivation of the effective Liouvillians for nonquadratic problems.

\section{APPENDIX D: SCHMUTZ'S FERMIONIC SUPEROPERATORS}

In Refs. 33 and 34, alternative field superoperators denoted by $a_{\sigma}, a_{\sigma}^{\dagger}$ and $\tilde{a}_{\sigma}, \tilde{a}_{\sigma}^{\dagger}$ were discussed, which were first introduced by Schmutz. ${ }^{32}$ In our notation (15)-(17), (39), and (40), they read as

$$
a_{1}^{p}=a_{\eta, \sigma}^{p}= \begin{cases}a_{\eta, \sigma}, & p=+1 \\ \tilde{a}_{\eta, \sigma}, & p=-1 .\end{cases}
$$

They are related to our "intermediate" superoperators (47) as follows (the definition of Ref. 34, in fact, contains an additional $p$ sign that is not relevant here):

$$
a_{\eta, \sigma}^{p}=p^{\frac{1-\eta}{2}} \mathcal{G}_{\eta, \sigma}^{p} .
$$

The use of the additional $\eta$-dependent factor $p^{\frac{1-\eta}{2}}$ allows one to compensate the inconvenient signs $p$ in the anticommutation relations (49) and in the Hermitian-conjugation relation (B4), thus restoring the usual fermionic algebra without performing the Keldysh rotation (51):

$$
\left[a_{1}^{p_{1}}, a_{2}^{p_{2}}\right]_{+}=\delta_{1, \overline{2}} \delta_{p_{1}, p_{2}}, \quad\left(a_{1}^{p}\right)^{\dagger}=a_{\overline{1}}^{p} .
$$

The spin and particle-hole group transformations of these superoperators coincide with those introduced by us. However, they do not reveal the important property (55) which is crucial in our formulation of the causal structure. Also, the explicit $\eta$ dependence of the sign prefactor does not allow one to perform a simple Keldysh rotation of the $a_{1}^{p}$ superoperators to recover this property. Finally, we mention that during the preparation of this paper, a work ${ }^{35}$ appeared, in which Schmutz's operators are modified by a complex phase factor.

\section{APPENDIX E: FERMION-PARITY OPERATOR AND SUPEROPERATOR}

In Sec. II C2, the operator $Z_{R}$ was constructed as the right eigensupervector $\mid Z_{R}$ ) of causal field superoperator $\bar{G}_{1}$ [cf. Eq. (86)] and turned out to play an important role. Here, we discuss its additional properties to further clarify its physical meaning.

Fermion parity. The causal field superoperators (51), written out in terms of field operators, read as

$$
G_{1}^{q} \bullet=\frac{1}{\sqrt{2}}\left\{d_{1} \bullet+q(-1)^{n} \bullet d_{1}(-1)^{n}\right\}
$$

where we used $(-1)^{L^{n}} \bullet=(-1)^{n} \bullet(-1)^{n}$. Note that $n=$ $\sum_{\sigma} n_{\sigma}$ is the occupation operator with $n_{\sigma}=d_{\sigma}^{\dagger} d_{\sigma}$. The definition of this representation is based on the fermion-parity superselection rule of quantum mechanics (cf. Sec. II B2). The explicit form (E1) makes clear that $G_{1}^{-}=\bar{G}_{1}$ has the operator

$$
Z_{R}=\frac{1}{2}(-1)^{n}
$$

as its right zero eigensupervector: upon substitution, the two terms in Eq. (E1) simply cancel since $(-1)^{2 n}=1$. Noting that $(-1)^{n}=\prod_{\sigma} e^{i \pi n_{\sigma}}=\prod_{\sigma}\left(1-2 n_{\sigma}\right)$, we recover the result $(90)$ in the main text:

$$
Z_{R}=\frac{1}{2}\left(2 n_{\uparrow}-1\right)\left(2 n_{\downarrow}-1\right)=2 \hat{n}_{\uparrow} \hat{n}_{\downarrow}-\hat{n}+\frac{1}{2} .
$$

Clearly, from Eq. (E2) it follows that

$$
Z_{R}^{2}=\frac{1}{4} \mathbb{1}
$$

which implies the normalization $\left(Z_{R} \mid Z_{R}\right)=1$. The eigenvalue equation (86), $\left.\bar{G}_{1} \mid Z_{R}\right)=\left[d_{1}, Z_{R}\right]_{+}=0$, requires $Z_{R}$ to anticommute with all the fermionic fields: $Z_{R}$ is therefore the unique operator (up to normalization and a phase) that (anti)commutes with all QD bosonic (fermionic) operators in the QD Liouville space, in close analogy to Grassmann numbers used in functional integral approaches. ${ }^{46}$ Equation (E2) most clearly illustrates the physical meaning of the operator $Z_{R}$ : we identify $Z_{R}$ as the fermion-parity operator (up to a constant)

$$
2 Z_{R}|x\rangle= \pm|x\rangle
$$

when the state $|x\rangle$ has a well-defined, even-/odd-fermion number. Finally, it follows from $(-1)^{n} d_{1}(-1)^{n}=-d_{1}$ that $\left.\mid Z_{L}\right)=\frac{1}{2} \mathbb{1}$ is a zero eigensupervector of $G_{1}^{-}$. The eigenvalue 
equation (85), $\left.\tilde{G}_{1} \mid Z_{L}\right)=\left[d_{1}, Z_{L}\right]_{-}=0$, requires $Z_{L}$, considered as an operator, to commute with the QD field operators and therefore with all QD operators. This implies that indeed $Z_{L}$ is proportional to the unit operator.

Spin and charge rotations. By construction, the two independent operators $Z_{L}$ and $Z_{R}$ transform as scalars under both spin and charge rotations (cf. Table I). They must therefore be related to the two scalar operators with respect to these groups, the Casimir operators of their Lie algebras $S^{2}=\sum_{i} S_{i}^{2}=$ $3 / 4 \sum_{\sigma}|\sigma\rangle\langle\sigma|$ and $T^{2}=\sum_{i} T_{i}^{2}=3 / 4(|0\rangle\langle 0|+| 2\rangle\langle 2|)$ :

$$
Z_{L}=\frac{1}{2} \mathbb{1}=\frac{2}{3}\left(T^{2}+S^{2}\right), \quad Z_{R}=\frac{2}{3}\left(T^{2}-S^{2}\right) .
$$

The first relation follows from the completeness relation and second one from Eq. (E5).

Fermion-parity superoperator. We next consider the fermion-parity superoperator, defined naturally by the right action of the fermion-parity operator on an operator (identical results follow for the left action):

$$
U \bullet:=\bullet 2 Z_{R}=\bullet(-1)^{n} .
$$

This unitary and Hermitian superoperator $\left(U^{\dagger}=U, U^{2}=\mathbb{1}\right)$ transforms the two types of causal field superoperators into each other,

$$
U G_{1}^{q} U=G_{1}^{\bar{q}} \text {. }
$$

We can thus interchange the role of creation and annihilation superoperators in Liouville Fock space by a linear transformation $U$. This is similar to the field operators $d_{\sigma}$ and $d_{\sigma}^{\dagger}$ that generate the standard Fock space: the $\eta$ index distinguishing can be inverted by a unitary transformation

$$
e^{i \pi\left(L_{T_{y}}-L_{S_{y}}\right)} d_{\eta, \sigma}=e^{i \pi\left(T_{y}-S_{y}\right)} d_{\eta, \sigma} e^{-i \pi\left(T_{y}-S_{y}\right)}=d_{\bar{\eta}, \sigma}
$$

[cf. Eqs. (107) and (110)]. Note that $K d_{\eta \sigma}=d_{\bar{\eta} \sigma}$ as well [cf. Eq. (G1)], but this is an antiunitary transformation.

The result (E8) follows by considering an arbitrary fermionic operator $F$ for which $(-1)^{L^{n}} F=(-1)^{n} F(-1)^{n}=$ $-F$. The superoperator $U$ transforms a commutator of $F$ with any operator to an anticommutator and vice versa: defining $L_{F}^{ \pm} \bullet=[F, \bullet]_{ \pm}=F \bullet \pm \bullet F:$

$$
\begin{aligned}
U L_{F}^{ \pm} U \bullet & =F \bullet(-1)^{2 n} \pm \bullet(-1)^{n} F(-1)^{n} \\
& =F \bullet \mp \bullet F=L_{F}^{\mp} \bullet .
\end{aligned}
$$

Commutators with any bosonic operator $B$ remain unaffected. Since the superoperator $\bar{G}_{\sigma}$ is a (anti)commutator when acting on a fermionic (bosonic) operator and vice versa for $\tilde{G}$, the superoperator $U$ interchanges these two: $U \bar{G}_{1} U=\tilde{G}_{1}$ and $U \tilde{G}_{1} U=\bar{G}_{1}$.

Multiorbital Anderson models. Finally, we indicate how the operators $\left.\mid Z_{L}\right)$ and $\left.\mid Z_{R}\right)$ can be constructed for more general multiorbital Anderson-type models. The super vacuum state is

$$
\left.\mid Z_{L}\right)=\frac{1}{2^{N}} \mathbb{1},
$$

where $N$ is the number of orbitals and the prefactor takes into account normalization $\left(Z_{L} \mid Z_{L}\right)=1$. Equation (87) is then simply extended to the maximally occupied state with respect to this vacuum

$$
\left.\left.\mid Z_{R}\right)=\prod_{k=1}^{N} \prod_{\sigma}\left(\prod_{\eta} \bar{G}_{\eta \sigma}^{k}\right) \mid Z_{L}\right) .
$$

Using Eqs. (E12) and (89), we can generalize Eq. (90) to

$$
\left.\mid Z_{R}\right)=\frac{1}{2^{N}} \prod_{k} \prod_{\sigma}\left(2 n_{\sigma}^{k}-\mathbb{1}\right)=\frac{1}{2^{N}} e^{i \pi n},
$$

implying $Z_{R}^{2}=\frac{1}{4^{N}} \mathbb{1}$. Here, $n=\sum_{k, \sigma} n_{\sigma}^{k}$ is the total dot particle-number operator. All properties of the single-orbital $Z_{R}$ operator, i.e., (anti)commutation relations with fermionic (bosonic) operators, transformation properties under charge and spin rotations, etc., also hold for the multiorbital case.

\section{APPENDIX F: SYMMETRY OF THE SELF-ENERGY}

In this Appendix, we derive the symmetry for QD Eq. (101) in contact with the reservoirs from the global symmetries (25) and (26). Quite generally, a quantity with QD and reservoir contributions $A^{\text {tot }}=A+A^{\mathrm{R}}$ is globally conserved when $\left[A^{\text {tot }}, H^{\text {tot }}\right]_{-}=0$. The corresponding Liouville superoperators $L^{A^{\text {tot }}}=\left[A^{\text {tot }}, \bullet\right]_{-}$and $L^{\text {tot }}=\left[H^{\text {tot }}, \bullet\right]_{-}$, then also commute:

$$
\begin{aligned}
& {\left[L^{A^{\mathrm{R}}}, L^{\mathrm{R}}\right]_{-}=0,} \\
& {\left[L^{A^{\mathrm{tot}}}, L^{\mathrm{tot}}\right]_{-}=0 .}
\end{aligned}
$$

The commutator of the local part $L^{A}=[A, \bullet]_{-}$with the Laplace-transformed evolution superoperator $\Pi(z)=$ $\operatorname{Tr}_{\mathrm{R}} i\left(z-L^{\mathrm{tot}}\right)^{-1} \rho^{\mathrm{R}}$ of the reduced density operator [cf. Eq. (31)] must then vanish:

$$
\begin{aligned}
L^{A} \Pi(z) & =L^{A} \underset{\mathrm{R}}{\operatorname{Tr}} \frac{i}{z-L^{\mathrm{tot}}} \rho^{\mathrm{R}}=\operatorname{Tr}_{\mathrm{R}} L^{A^{\mathrm{tot}}} \frac{i}{z-L^{\mathrm{tot}}} \rho^{\mathrm{R}} \\
& =\operatorname{Tr}_{\mathrm{R}} \frac{i}{z-L^{\mathrm{tot}}} L^{A} \rho^{\mathrm{R}}=\Pi L^{A}
\end{aligned}
$$

using subsequently $\operatorname{Tr}_{\mathrm{R}} L^{A^{\mathrm{R}} \bullet} \bullet \operatorname{Tr}_{\mathrm{R}}\left[H^{\mathrm{R}}, \bullet\right]_{-}=0$ [Eq. (F2)], and finally $L^{A^{\mathrm{R}}} \rho^{\mathrm{R}}=0$. We note that this last result follows from Eq. (21) under the assumption that $A^{\mathrm{R}}$ conserves both the reservoir energy and the particle number $\left[A^{\mathrm{R}}, H^{\mathrm{R}}\right]_{-}=$ $\left[A^{\mathrm{R}}, n^{\mathrm{R}}\right]_{-}=0$, which is the case in our applications of the result. Clearly, this proof applies also to the time-evolution superoperator $\Pi_{0}$ without any QD reservoir interaction, i.e., for $L^{V}=0$. Then, using $\left[L^{A}, \Pi(z)\right]_{-}=\left[L^{A}, \Pi_{0}(z)\right]_{-}=0$ and taking the commutator of $L^{A}$ with the Dyson equation that defines the self-energy $\Sigma(z), \Pi(z)=\Pi_{0}(z)-i \Pi_{0}(z) \Sigma(z) \Pi(z)$, we find that $\left[L^{A}, \Sigma(z)\right]_{-}=0$.

\section{APPENDIX G: HERMITICITY}

Hermitian conjugation superoperator. The density operator is restricted to be invariant under Hermitian conjugation in Hilbert space. When considering density operators as supervectors in Liouville space, this Hermitian conjugation of an operator then corresponds to a superoperator that we denote by $K$ :

$$
\left.K \mid A):=\mid A^{\dagger}\right) \text {. }
$$

It is to be distinguished from the Hermitian conjugation of a superoperator. $K$ is antilinear and satisfies

$$
K^{2}=\mathcal{I}
$$

where $\mathcal{I}$ is the unit superoperator. Changing the basis in Liouville space by the superoperator $K$, we effect an antilinear 
transformation of a superoperator $S \rightarrow K S K$, referred to as " $c$ transform" in Ref. 26. In the time representation, the density operator is invariant under this transformation: $K \rho(t)=\rho(t)$, implying for the Laplace-transformed density matrix (32) $K \rho(z)=\rho\left(-z^{*}\right)$. Applying $K$ to the kinetic equation (35), we obtain a conjugation relation restricting the kernel $\Sigma(z)$ :

$$
K \Sigma(z) K=-\Sigma\left(-z^{*}\right) .
$$

Of course, this property also holds for the initial Liouvillian: $K L K \bullet=\left[H, \bullet^{\dagger}\right]_{-}^{\dagger}=-\left[H^{\dagger}, \bullet\right]_{-}=-[H, \bullet]_{-}=-L \bullet$.

The transformation of the fields $\mathscr{G}^{p}$ follows by applying $K$ to Eq. (40) and using $\left(d^{\dagger} \bullet\right)^{\dagger}=\bullet^{\dagger} d=(K \bullet) d$,

$$
K \mathscr{G}_{1}^{p} K=\mathscr{G}_{\overline{1}}^{\bar{p}}
$$

giving with Eqs. (47), (G2), and $K L^{n} K=-L^{n}$ :

$$
K \mathcal{G}_{1}^{p} K=p^{-L^{n}} \mathscr{G}_{\overline{1}}^{\bar{p}}=(-1)^{L^{n}} \mathcal{G}_{\overline{1}}^{\bar{p}} .
$$

The transformation of the causal field superoperators $G^{q}$ [Eq. (119) in the main text] now follows by applying Eq. (51):

$$
K G_{1}^{q} K=q(-1)^{L^{n}} G_{\overline{1}}^{q} .
$$

Simplifications using conjugation. The transformation behavior of the kernel $\Sigma(z)$ under $K$ conjugation of the basis vectors $K \bar{\Sigma}(z) K=-\bar{\Sigma}\left(-z^{*}\right)$ restricts the structure of the contributions to $\bar{\Sigma}(z)$ in the renormalized perturbation theory (79). The RG equations for the Liouvillian (206), (222), and (267) have a similar structure [since we can eliminate the renormalized vertices, even in the two-loop RG approximation, we can restrict our considerations to the bare vertices $\bar{G}$ as in Eq. (79)]. To make use of this, we decompose $\bar{\Sigma}(z)$ into conjugate pairs of contributions. To illustrate the idea, consider first the one-loop approximation to the perturbation theory (79) for $\bar{\Sigma}(z)$ :

$$
\bar{\Sigma}(z)=\sum_{1} \bar{\Sigma}_{1 \overline{1}}(z) .
$$

Here, we write the sum over 1 explicitly and let $\bar{\Sigma}_{1 \overline{1}}(z):=$ $\bar{\gamma}\left(x_{1}\right) \bar{G}_{1} \Pi_{1} \bar{G}_{\overline{1}}=-K \bar{\Sigma}_{\overline{1} 1}\left(-z^{*}\right) K$ denote a term in which the multi-index 1 has a fixed value and $\Pi_{1}=\left(z-\bar{L}-x_{1}\right)^{-1}$. Furthermore, noting that 1 is a dummy multi-index, we can restrict the summation to one fixed $\eta$ configuration, e.g., $\eta_{1}=+$, while manifestly preserving the structure $K \bar{\Sigma}(z) K=-\bar{\Sigma}\left(-z^{*}\right)$ :

$$
\bar{\Sigma}(z)=\sum_{1} \delta_{\eta_{1}+}\left(\bar{\Sigma}_{1 \overline{1}}(z)-K \bar{\Sigma}_{1 \overline{1}}\left(-z^{*}\right) K\right) .
$$

The calculation of the supermatrix elements is now simplified: using the notation of Sec. III and the antilinearity of $K$, $(A|K S K| B)=(K A|S| K B)^{*}$, we obtain

$$
\begin{aligned}
& {\left[\kappa_{3}|\bar{\Sigma}(z)| \kappa_{0}\right]} \\
& \quad=\sum_{1} \delta_{\eta_{1}+}\left[\left(\kappa_{3}\left|\bar{G}_{1}\right| \kappa_{2}\right)\left(\kappa_{1}\left|\bar{G}_{\overline{1}}\right| \kappa_{0}\right)\left(\kappa_{2}\left|\Pi_{1}(z)\right| \kappa_{1}\right)\right. \\
& \left.\quad-\left(K \kappa_{3}\left|\bar{G}_{1}\right| \kappa_{2}\right)^{*}\left(\kappa_{1}\left|\bar{G}_{\overline{1}}\right| K \kappa_{0}\right)^{*}\left(\kappa_{2}\left|\Pi_{1}\left(-z^{*}\right)\right| \kappa_{1}\right)^{*}\right] .
\end{aligned}
$$

In our Liouville Fock basis, the matrices representing $G^{q}$ are real, just as those of the field operators $d_{1}$ [cf. Eq. (E1)]. When the basis supervectors $\left.\left.\mid \kappa_{3}\right), \mid \kappa_{0}\right)$ correspond to diagonal operators $\left(Z_{i}, \chi_{\sigma}, S_{0}, T_{0}\right)$, then the second term in Eq. (G9) simply relates to the first one since these supervectors are mapped onto themselves by $K$ [cf. Eq. (116)]. Equation (G9) simplifies to

$$
\begin{aligned}
{\left[\kappa_{3}|\bar{\Sigma}(z)| \kappa_{0}\right]=} & \sum_{1} \delta_{\eta_{1}+}\left(\kappa_{3}\left|\bar{G}_{1}\right| \kappa_{2}\right)\left(\kappa_{1}\left|\bar{G}_{\overline{1}}\right| \kappa_{0}\right) \\
& \times\left[\left(\kappa_{2}\left|\Pi_{1}(z)\right| \kappa_{1}\right)-\left(\kappa_{2}\left|\Pi_{1}\left(-z^{*}\right)\right| \kappa_{1}\right)^{*}\right],
\end{aligned}
$$

which is explicitly imaginary at $z=i 0$ as it should be. Supervectors corresponding to nondiagonal operators $\left(S_{\sigma}, T_{\eta}, \alpha_{\eta \sigma}^{v}\right)$ come in pairs related by inversion of both indices $\eta$ and $\sigma$. The superoperator $K$ maps these pairs onto each other [possibly with a sign change, see Eq. (117)]. Noting also that $\bar{G}$ only has nonzero matrix elements for one superket of each pair [cf. Eqs. (118)-(120)], we see that in Eq. (G8) either the first or second term contributes or neither when $\kappa_{3}$ and/or $\kappa_{0}$ is a nondiagonal operator. In this way we have effectively eliminated the need to evaluate the terms for $\eta_{1}=-$ using the conjugation relations. This consideration is generalized to two-loop expressions by adding to Eq. (G8)

$$
\sum_{12} \delta_{\eta_{1}+} \delta_{\eta_{2}+}\left(\bar{\Sigma}_{1 \overline{1} 2 \overline{2}}(z)-K \bar{\Sigma}_{1 \overline{1} 2 \overline{2}}\left(-z^{*}\right) K\right),
$$

where $\bar{\Sigma}_{1 \overline{1} 2 \overline{2}}(z)$ collects all two-loop terms with fixed multi-indices 1 and 2 . The same analysis applies to terms in the RG equations (206), (222), and (267).

Conjugation properties under RG flow. In contrast to the charge- and spin-transformation properties, the conjugation property (G6), associated with the fundamental Hermiticity of the density operator, is exactly preserved under the RG flow. We first note that the vertices $\tilde{G}_{1}=G_{1}^{+}$only determine the initial value of the Liouvillian $\bar{L}$ and therefore are not affected by the ensuing RG flow: they therefore simply obey Eq. (G6). However, the vertices $\bar{G}_{1}=G_{1}^{-}$flow together with the Liouvillian $\bar{L}$ and thereby acquire a dependence on the dot frequency $z$. These vertices obey the following generalization of Eq. (G6) to nonzero $z$ :

$$
K G_{1}^{q}(z) K=q(-1)^{L^{n}} G_{1}^{q}\left(-z^{*}\right) .
$$

To prove Eq. (G12), we use that the bare vertices, providing the initial values of the RG flow, have the property (G12). It remains to show that for each scale $\Lambda$ the infinitesimal correction to the $\bar{G}$ generated by the RG flow also has this property. We therefore apply $K \bullet K$ to the RG equation (207) for the vertex $\bar{G}_{\alpha}$, insert Eq. (G2), use Eq. (G3) and assume that the property (G12) holds:

$$
\begin{aligned}
& K \frac{d \bar{G}_{\alpha}}{d \Lambda} K=(-1)^{(k+1) L^{n}+k(k+1) / 2}\left(\frac{d \bar{\gamma}}{d \Lambda} \prod_{i} \bar{\gamma}_{i}\left(\bar{\omega}_{i}\right)\right)_{\mathrm{irr}} \\
& \times \bar{G}_{\overline{1}} \frac{1}{z_{1}^{*}+\bar{L}\left(-z_{1}^{*}\right)} \bar{G}_{\overline{2}} \ldots \bar{G}_{\bar{\alpha}} \ldots \bar{G}_{\overline{k-1}} \frac{1}{z_{l}^{*}+\bar{L}\left(-z_{l}^{*}\right)} \bar{G}_{\bar{k}} \\
= & (-1)^{L^{n}}\left(\frac{d \bar{\gamma} 13)}{d \Lambda} \prod_{i} \bar{\gamma}_{i}\left(\bar{\omega}_{i}\right)\right)_{\mathrm{irr}} \\
& \times \bar{G}_{1} \frac{\bar{\gamma}\left(\bar{\omega}_{1}\right)}{-z_{1}^{*}-\bar{L}_{\left(-z_{1}^{*}\right)}} \bar{G}_{2} \ldots \bar{G}_{\alpha} \ldots \bar{G}_{k-1} \frac{\bar{\gamma}\left(\bar{\omega}_{l}\right)}{-z_{l}^{*}-\bar{L}\left(-z_{l}^{*}\right)} \bar{G}_{k} \\
= & -(-1)^{L^{n}} \frac{d \bar{G}_{\alpha}}{d \Lambda}\left(-z^{*}\right) .
\end{aligned}
$$


At the first equality, a sign factor arises when commuting all $(-1)^{L^{n}}$ factors to the left, using the fermion-parity property $(-1)^{L^{n}} \bar{G}_{1}=\bar{G}_{1}(-1)^{L^{n}+1}$ which is preserved under the RG as well [since both sides of Eq. (207) have an odd number of $\bar{G}$ 's]. After the second equality, we inverted all dummy multi-indices $i \rightarrow \bar{i}$ and inverted the integration variable $\bar{\omega}_{i} \rightarrow-\bar{\omega}_{i}$, giving a sign $(-1)^{k / 2}$ due to the $k / 2$ antisymmetric contraction functions (counting $\bar{\gamma}$ 's and $d \bar{\gamma} / d \Lambda$ ), where $k$ is the even number of vertices other than $\bar{G}_{\alpha}$. Since for even $k$ the integers $k / 2$ and $k(k+1) / 2$ have opposite parity, the result follows.
${ }^{1}$ L. I. Glazman and M. E. Raikh, JETP Lett. 47, 452 (1988) [Pis'ma v ZhETF 47, 378 (1988)].

${ }^{2}$ T. K. Ng and P. A. Lee, Phys. Rev. Lett. 61, 1768 (1988).

${ }^{3}$ R. Bulla, T. Costi, and T. Pruschke, Rev. Mod. Phys. 80, 395 (2008).

${ }^{4}$ J. Eckel, F. Heidrich-Meisner, S. Jakobs, M. Thorwart, M. Pletyukhov, and R. Egger, New J. Phys. 12, 043042 (2010).

${ }^{5}$ F. B. Anders, Phys. Rev. Lett. 101, 066804 (2008).

${ }^{6}$ A. Rosch, Euro. Phys. J. 85, 6 (2012).

${ }^{7}$ S. R. White and A. E. Feiguin, Phys. Rev. Lett. 93, 076401 (2004).

${ }^{8}$ A. Daley, C. Kollath, U. Schollwöck, and G. Vidal, J. Stat. Mech.: Theor. Exp. (2004) P04005.

${ }^{9}$ P. Schmitteckert, Phys. Rev. B 70, 121302 (2004).

${ }^{10} \mathrm{~S}$. Weiss, J. Eckel, M. Thorwart, and R. Egger, Phys. Rev. B 77, 195316 (2008).

${ }^{11}$ D. Segal, A. J. Millis, and D. R. Reichman, Phys. Rev. B 82, 205323 (2010).

${ }^{12}$ D. Segal, A. J. Millis, and D. R. Reichman, Phys. Chem. Chem. Phys. 13, 14378 (2011).

${ }^{13}$ G. Cohen and E. Rabani, Phys. Rev. B 84, 075150 (2011).

${ }^{14}$ J. E. Han, Phys. Rev. B 81, 245107 (2010).

${ }^{15}$ L. Mühlbacher, D. F. Urban, and A. Komnik, Phys. Rev. B 83, 075107 (2011).

${ }^{16}$ N. S. Wingreen and Y. Meir, Phys. Rev. B 49, 11040 (1994).

${ }^{17}$ K. Haule, S. Kirchner, J. Kroha, and P. Wölfle, Phys. Rev. B 64, 155111 (2001).

${ }^{18}$ R. VanRoermund, S. Y. Shiau, and M. Lavagna, Phys. Rev. B 81, 165115 (2010).

${ }^{19}$ P. Mehta and N. Andrei, Phys. Rev. Lett. 96, 216802 (2006).

${ }^{20}$ R. M. Konik, H. Saleur, and A. W. Ludwig, Phys. Rev. B 66, 125304 (2002).

${ }^{21}$ P. Wang and S. Kehrein, Phys. Rev. B 82, 125124 (2010).

${ }^{22}$ A. Oguri, Phys. Rev. B 64, 153305 (2001).

${ }^{23}$ A. A. Aligia, Phys. Rev. B 74, 155125 (2006).

${ }^{24}$ E. Munoz, C. Bolech, and S. Kirchner, Phys. Rev. Lett. (to be published), arXiv:1111.4076.

${ }^{25}$ J. Paaske, A. Rosch, and P. Wölfle, Phys. Rev. B 69, 155330 (2004).

${ }^{26}$ H. Schoeller, Eur. Phys. J. B 168, 179 (2009).

${ }^{27}$ H. Schoeller and F. Reininghaus, Phys. Rev. B 80, 045117 (2009).

${ }^{28}$ H. Schoeller and G. Schön, Phys. Rev. B 50, 18436 (1994).

${ }^{29}$ S. Koller, M. Grifoni, M. Leijnse, and M. R. Wegewijs, Phys. Rev. B 82, 235307 (2010).

${ }^{30}$ M. Leijnse and M. R. Wegewijs, Phys. Rev. B 78, 235424 (2008).

${ }^{31}$ T. Prosen, New J. Phys. 10, 043026 (2008).

${ }^{32}$ M. Schmutz, Z. Phys. B: Condens. Matter Quanta 30, 97 (1978).

${ }^{33}$ A. Dzhioev and D. Kosov, J. Chem. Phys. 134, 044121 (2011).

${ }^{34}$ U. Harbola and S. Mukamel, Phys. Rep. 465, 191 (2008).

${ }^{35}$ A. A. Dzhioev and D. S. Kosov, J. Phys.: Condens. Matter 24, 225304 (2012).

${ }^{36}$ S. Andergassen, M. Pletyukhov, D. Schuricht, H. Schoeller, and L. Borda, Phys. Rev. B 83, 205103 (2011).
${ }^{37}$ J. König, H. Schoeller, and G. Schön, Phys. Rev. Lett. 76, 1715 (1996).

${ }^{38}$ F. Reckerman, M. Wegewijs, J. Splettstoesser, and R. Saptsov (unpublished).

${ }^{39}$ L. D. Contreras-Pulido, J. Splettstoesser, M. Governale, J. König, and M. Büttiker, Phys. Rev. B 85, 075301 (2012).

${ }^{40}$ To treat the reservoir and dot field operators in the same way, our $\eta$ sign convention is opposite to that of Ref. 26.

${ }^{41}$ This gives the correct grand-canonical average over the reservoirs (see Appendix A in Ref. 26 for a proof).

${ }^{42}$ G. C. Wick, A. S. Wightman, and E. P. Wigner, Phys. Rev. 88, 101 (1952).

${ }^{43}$ Y. Aharonov and L. Susskind, Phys. Rev. 155, 1428 (1967).

${ }^{44}$ S. Mukamel, Phys. Rev. E 68, 021111 (2003).

${ }^{45}$ A. I. Larkin and Y. Ovchinnikov, Zh. Eksp. Teor. Fiz. 68, 1915 (1975) [Sov. Phys. JETP 41, 960 (1975)].

${ }^{46}$ A. Kamenev and A. Levchenko, Adv. Phys. 58, 197 (2009).

${ }^{47}$ M. Esposito, U. Harbola, and S. Mukamel, Rev. Mod. Phys. 81, 1665 (2009).

${ }^{48}$ E. Lifshitz and L. Pitaevskiı̆, Physical Kinetics (ButterworthHeinemann, Oxford, UK, 1981).

${ }^{49}$ Formulating the theory in real time (Ref. 38), one can directly identify the retarded and Keldysh Green's functions with their usual definitions (Ref. 53) when setting $\Gamma=$ $\pi$ [cf. Eq. (19)], $-i\left\langle\mathcal{T} \bar{J}_{1}(t) \tilde{J}_{2}\right\rangle_{\mathrm{R}}=-i \theta(t)\left\langle\left[b_{1}(t), b_{2}\right]_{+}\right\rangle_{\mathrm{R}}$, and $-i\left\langle\mathcal{T} \bar{J}_{1}(t) \bar{J}_{2}\right\rangle_{\mathrm{R}}=-i\left\langle\left[b_{1}(t), b_{2}\right]_{-}\right\rangle_{\mathrm{R}}$ where $\mathcal{T}$ is the time-ordering superoperator. In Laplace space, their time-evolution frequencies $X$ are convoluted together with the dot Liouvillian into $1 /(z-X-L)$ in the diagram rules (68). The retarded function $\left\langle\mathcal{T} \bar{J}_{1}(t) \tilde{J}_{2}\right\rangle_{\mathrm{R}}$ thus contributes only spectral information through $X$ since Eq. (63) contains only $\left\langle\bar{J}_{1} \tilde{J}_{2}\right\rangle_{\mathrm{R}} \propto\left\langle\left[b_{1}, b_{2}\right]_{+}\right\rangle_{\mathrm{R}}=\delta_{1,2}$, whereas the Keldysh function $-i\left\langle\mathcal{T} \bar{J}_{1}(t) \bar{J}_{2}\right\rangle_{\mathrm{R}}$ additionally includes statistical information through Eq. (64) containing $\left\langle\bar{J}_{1} \bar{J}_{2}\right\rangle_{\mathrm{R}} \propto\left\langle\left[b_{1}, b_{2}\right]_{-}\right\rangle_{\mathrm{R}}$.

${ }^{50}$ R. B. Saptsov and M. R. Wegewijs (unpublished).

${ }^{51}$ In the wide-band limit, the energy-independent contractions $\tilde{\gamma}$ correspond to the $\delta$ functions in time, leaving no phase space for the $\omega$ integrals when enclosing vertices.

${ }^{52}$ J. König, J. Schmid, H. Schoeller, and G. Schön, Phys. Rev. B 54, 16820 (1996).

${ }^{53}$ J. Rammer and H. Smith, Rev. Mod. Phys. 58, 323 (1986).

${ }^{54}$ For models not of the Anderson type (bilinear coupling), higher skeleton diagrams and vertex renormalizations arise, requiring a more general discrete renormalization (Ref. 26).

${ }^{55}$ T. Korb, F. Reininghaus, H. Schoeller, and J. König, Phys. Rev. B 76, 165316 (2007).

${ }^{56}$ S. Mukamel, Phys. Rep. 93, 1 (1982).

${ }^{57}$ This relies on the anticommutation relations (53) of the bare vertices, which break down under renormalization: $\left.\mid Z_{R}\right)$ given by Eq. (87) is thus not an eigensupervector of renormalized vertices.

${ }^{58} \mathrm{We}$ emphasize that charge off-diagonal dot operators must be taken into account since in a virtual intermediate state of an open system, 
where, e.g., $G$ has acted on a even-fermion-parity QD density operator, there is a corresponding fermion operator $J$ acting on the reservoir. Although the latter is no longer explicitly present in the reduced density operator description (the reservoirs are integrated out), there is no change of the fermion parity of the total system described by an elementary process in $\Sigma$, in full agreement with the discussion of Eq. (38).

${ }^{59}$ Our definition of the contraction $\bar{\gamma}_{\Lambda}$ differs from that in Ref. 26 by a factor $-2 \Gamma$ due to our (i) our sign convention for the $\eta$ index (Ref. 40) [cf. Eq. (15) (factor -1)], (ii) our normalization of the field superoperators (Ref. 61) such that $\left[\bar{G}_{\overline{1}}, \tilde{G}_{1}\right]_{+}=1$ (factor 2 ), and (iii) our inclusion of the coupling into the reservoir fields (factor $\Gamma$ ).

${ }^{60}$ The positive imaginary part (integration contour in the lower halfplane) contrasts with Ref. 26, but the final notation (202) agrees again with Ref. 26 due to compensating differences in the notation.

${ }^{61}$ Equations (206) and (207) differ from those in Ref. 26 by a factor 2 for each loop due to our different normalization (Ref. 59) of $\bar{G}$ (reduced by factor $\sqrt{2}$ ) The sign difference in the definition of the cutoff function (Ref. 59) is compensated by our closing of the integration-contour (Ref. 60) in the lower (instead of the upper) half-plane.

${ }^{62}$ For the density operator time evolution, we need the Liouvillian $L, L+\Sigma(z)=\bar{L}+\bar{\Sigma}(z)=\bar{L}_{\Lambda}+\bar{\Sigma}_{\Lambda}(z)$, but for the current we do not: $\Sigma^{r}(z)=\tilde{\Sigma}^{r}+\bar{\Sigma}^{r}(z)=\bar{L}_{\Lambda}^{r}+\bar{\Sigma}_{\Lambda}^{r}(z)$.

${ }^{63} \mathrm{H}$. Schoeller, Mesoscopic Electron Transport (Kluwer, Dordrecht, 1997), p. 291.
${ }^{64} \mathrm{H}$. Schoeller, Interactions and Transport Properties (Springer, Berlin, 1999), p. 137.

${ }^{65}$ M. Pletyukhov and H. Schoeller, Phys. Rev. Lett. 108, 260601 (2012).

${ }^{66}$ K. Goß, S. Smerat, M. Leijnse, M. R. Wegewijs, C. M. Schneider, and C. Meyer, Phys. Rev. B 83, 201403(R) (2011).

${ }^{67}$ A. Eliasen, J. Paaske, K. Flensberg, S. Smerat, M. Leijnse, M. R. Wegewijs, H. I. Jørgensen, M. Monthioux, and J. Nygård, Phys. Rev. B 81, 155431 (2010).

${ }^{68}$ S. J. Tans, M. H. Devoret, R. J. A. Groeneveld, and C. Dekker, Nature (London) 394, 761 (1998).

${ }^{69}$ T. H. Oosterkamp, J. W. Janssen, L. P. Kouwenhoven, D. G. Austing, T. Honda, and S. Tarucha, Phys. Rev. Lett. 82, 2931 (1999).

${ }^{70}$ S. M. Reimann, M. Koskinen, M. Manninen, and B. R. Mottelson, Phys. Rev. Lett. 83, 3270 (1999).

${ }^{71}$ S. M. Reimann and M. Manninen, Rev. Mod. Phys. 74, 1283 (2002).

${ }^{72}$ J. Lambe and R. C. Jaklevic, Phys. Rev. 165, 821 (1968).

${ }^{73}$ M. Eto, Jpn. J. Appl. Phys. 40, 1929 (2001).

${ }^{74}$ J. Paaske, A. Rosch, P. Wölfle, N. Mason, C. M. Marcus, and J. Nygard, Nat. Phys. 2, 460 (2006).

${ }^{75}$ A. S. Zyazin, J. W. van den Berg, E. A. Osorio, H. S. van der Zant, N. P. Konstantinidis, F. May, M. Leijnse, W. Hofstetter, M. R. Wegewijs, C. Danieli et al., Nano Lett. 10, 3307 (2010).

${ }^{76}$ J. V. Holm, H. I. Jørgensen, K. Grove-Rasmussen, J. Paaske, K. Flensberg, and P. E. Lindelof, Phys. Rev. B 77, 161406 (2008).

${ }^{77}$ M. Gaudin, Nucl. Phys. 15, 89 (1960). 